ANALYSIS \& PDE

Volume $4 \quad$ No. $2 \quad 2011$

\title{
JACQUES SMULEVICI \\ ON THE AREA OF THE SYMMETRY ORBITS OF COSMOLOGICAL SPACETIMES WITH TOROIDAL OR HYPERBOLIC SYMMETRY
}




\title{
ON THE AREA OF THE SYMMETRY ORBITS OF COSMOLOGICAL SPACETIMES WITH TOROIDAL OR HYPERBOLIC SYMMETRY
}

\author{
JACQUES SMULEVICI
}

\begin{abstract}
We prove several global existence theorems for spacetimes with toroidal or hyperbolic symmetry with respect to a geometrically defined time. More specifically, we prove that generically, the maximal Cauchy development of $T^{2}$-symmetric initial data with positive cosmological constant $\Lambda>0$, in the vacuum or with Vlasov matter, may be covered by a global areal foliation with the area of the symmetry orbits tending to zero in the contracting direction. We then prove the same result for surface symmetric spacetimes in the hyperbolic case with Vlasov matter and $\Lambda \geq 0$. In all cases, there is no restriction on the size of initial data.
\end{abstract}

1. Introduction 191

2. Preliminaries 195

3. The maximal Cauchy development 198

4. Global areal foliations 198

5. The theorems 199

6. Remarks on the strategy of the proofs 200

7. Proof of Theorem $1 \quad 204$

8. Proof of Theorem 2 216

9. Proof of Theorem $3 \quad 235$

10. Comments and open questions 240

$\begin{array}{lll}\text { Appendix A. Initial data and the constraint equations } & 242\end{array}$

Appendix B. Surface-symmetric spacetimes in areal coordinates $\quad 243$

Appendix C. From symmetric initial data to symmetric spacetimes 243

Acknowledgements $\quad 244$

$\begin{array}{ll}\text { References } & 244\end{array}$

\section{Introduction}

The study of the global Cauchy problem constitutes one of the main areas of research in mathematical relativity and is one of the most natural problems to investigate in view of the hyperbolicity of the Einstein equations and of the theorems concerning the local Cauchy problem [Fourès-Bruhat 1952; ChoquetBruhat and Geroch 1969]. These theorems assert that given an appropriate initial data set, there exists a maximal solution of the Einstein equations

$$
R_{\mu \nu}-\frac{1}{2} g_{\mu \nu} R=8 \pi T_{\mu \nu}-\Lambda g_{\mu \nu},
$$

MSC2000: 83C05.

Keywords: Einstein equations, singularities, hyperbolic partial differential equations. 
coupled if necessary to appropriate matter equations, ${ }^{1}$ which is unique up to diffeomorphism in the class of globally hyperbolic spacetimes. We call this solution the maximal Cauchy development of the initial data. The global hyperbolicity assumption guarantees the domain of dependence property and is essential to the uniqueness statement.

The global Cauchy problem consists in understanding the global geometry of the maximal Cauchy development. A fundamental conjecture, known as strong cosmic censorship, ${ }^{2}$ states that the maximal Cauchy development of generic compact or asymptotically flat initial data is inextendible as a regular ${ }^{3}$ Lorentzian manifold. This can be thought of as a statement of uniqueness in a class of spacetimes not assumed a priori to be globally hyperbolic.

The expression "generic initial data" in the statement of the conjecture reflects the fact that there exist particular initial data for which the maximal Cauchy development fails to be inextendible. However, the extendibility property of the maximal Cauchy development for these particular initial data is expected to be dynamically unstable and, as we shall see below, this expectation has been verified in several cases. From the point of view of physics, uniqueness means predictability and thus, strong cosmic censorship asserts that, generically, general relativity is a deterministic theory in the same sense that Newtonian mechanics is deterministic.

1A. Areal foliations of $T^{2}$-symmetric and $k \leq 0$ surface-symmetric spacetimes. In full generality, the questions tied to the global Cauchy problem are not accessible with the current set of mathematical techniques. In order to make progress, one may try to look at simpler but connected problems, such as the study of the global Cauchy problem within certain classes of symmetries.

Following this approach, two classes of solutions arising from compact initial data with symmetry have been given much attention recently, the so-called $T^{2}$-symmetric and surface-symmetric spacetimes. The $T^{2}$-symmetric spacetimes constitute a class of solutions arising from initial data with spatial topology $T^{3}$ and admitting a torus action. They contain as special subcases the $T^{3}$-Gowdy spacetimes and the polarized $T^{2}$-symmetric spacetimes. ${ }^{4}$ The surface-symmetric spacetimes constitute a class of solutions arising from initial data where the initial Riemannian 3-manifold is given by a doubly warped product $S^{1} \times \mathscr{S}$, where $\mathscr{Y}$ is a compact 2 -surface of constant curvature $k$ and such that the rest of the initial data is invariant under the local isometries of $\mathscr{Y}$. By rescaling, $k$ may be taken as being $-1,0$ or +1 and the different cases are known as hyperbolic, plane ${ }^{5}$ or spherical symmetry.

In the case of $T^{2}$-symmetric or $k \leq 0$ surface-symmetric spacetimes, the local geometry of the solution possesses the particular property that, unless the spacetime is flat, the symmetry orbits are either trapped or antitrapped, a feature which is shared by the spheres of symmetry in the black or white hole regions of a Schwarzschild solution with $m>0$. If we denote by $t$ the area of the symmetry orbits, this means that

\footnotetext{
${ }^{1}$ See [Choquet-Bruhat 1970; 1971] for the case of Vlasov matter.

${ }^{2}$ The conjecture was originally developed by R. Penrose [1979] and first formulated as a statement about the global geometry of the maximal Cauchy development in [Moncrief and Eardley 1981]. See also the presentation in [Christodoulou 1999].

${ }^{3}$ The regularity concerns here the degree of differentiability of the possible extensions and gives rise to different versions of the conjecture. For instance, the $C^{2}$ formulation of the conjecture is obtained by replacing "regular" with $C^{2}$ in the above statement of the conjecture.

${ }^{4}$ They also contain the even more special case of polarized $T^{3}$-Gowdy spacetimes.

${ }^{5}$ Note that the plane symmetric case is a special case of $T^{3}$-Gowdy polarized solutions.
} 
the gradient of $t$ is everywhere timelike and that $t$ may be used as a time coordinate. ${ }^{6}$ For the vacuum $T^{2}$-symmetric case with zero cosmological constant $(\Lambda=0)$, the existence of a global areal foliation where $t$ takes value in $\left(t_{0}, \infty\right)$ with $t_{0} \geq 0$ was proven in [Berger et al. 1997]. The proof was then extended to the Vlasov case [Andréasson 1999; Andréasson et al. 2004] and to the case with $\Lambda>0$ [Clausen and Isenberg 2007]. Similarly, the existence of a global areal foliation for the surface-symmetric case with $k=-1, \Lambda=0$ and Vlasov matter ${ }^{7}$ was proven in [Andréasson et al. 2003] and extended to the case with $\Lambda>0$ in [Tchapnda and Rendall 2003; Tchapnda and Noutchegueme 2005].

It was soon realized that in the vacuum $T^{3}$-Gowdy case with $\Lambda=0$, one has $t_{0}=0$ unless the spacetime is flat [Moncrief 1981; Chruściel 1990]. The natural question arose: Is $t_{0}=0$ generically for all the possible cases? The proofs that $t_{0}=0$ generically for $T^{2}$-symmetric spacetimes with $\Lambda=0$, in the vacuum or with Vlasov matter, were given in [Isenberg and Weaver 2003] and [Weaver 2004]. It has also been proven that $t_{0}=0$ in the case of plane symmetric initial data with $\Lambda=0$ and Vlasov matter as well as in the case of plane or hyperbolic symmetric initial data with $\Lambda \geq 0$ and Vlasov matter under an extra small data assumption [Rein 1996; Tchapnda 2004]. Moreover, the results for $k \leq 0$ surfacesymmetric initial data have been extended to the Einstein-Vlasov-scalar field system [Tegankong and Rendall 2006].

1B. Strong cosmic censorship for $T^{2}$-symmetric or surface-symmetric spacetimes. One motivation for the study of the value of $t_{0}$ was the expectation that, in the cases were $t_{0}=0$, the curvature should in general blow up as $t$ goes to 0 , thus providing a proof of inextendibility (and thus of strong cosmic censorship) for these cases. Indeed, for vacuum $T^{3}$-Gowdy spacetimes with $\Lambda=0$, first in the polarized case $^{8}$ and then for the full class, detailed asymptotic expansions were obtained and used in this sense to establish a proof of the $C^{2}$ formulation of the strong cosmic censorship conjecture [Chruściel et al. 1990; Ringström 2006; 2009].

While it seemed difficult to extend the analysis of the vacuum $T^{3}$-Gowdy spacetimes to the more general case of $T^{2}$-symmetric spacetimes, strong cosmic censorship was nonetheless proven for $T^{2}$ symmetric spacetimes with $\Lambda=0$ in the presence of Vlasov matter [Dafermos and Rendall 2006]. The analysis starts with the remark that for $T^{2}$-symmetric or $k \leq 0$ surface-symmetric spacetimes, with or without Vlasov matter and with $\Lambda \geq 0$, the fact that $t$ is unbounded implies inextendibility in the expanding direction because of the continuous extension of the Killing fields to possible Cauchy horizons [Dafermos and Rendall 2005]. Thus it is sufficient to study the contracting direction in order to complete the proof of strong cosmic censorship for these classes of spacetimes. The proof given in [Dafermos and Rendall 2006] relied on a rigidity of the possible Cauchy horizon, linked with the fact that $t_{0}=0$, and on the particular properties of the Vlasov equation. The assumption that $\Lambda=0$ was necessary only as to ensure that $t_{0}=0$. Therefore the proof remained valid in the case where $\Lambda>0$, if one added

\footnotetext{
${ }^{6}$ In particular, any nonflat $T^{2}$-symmetric or $k \leq 0$ surface-symmetric spacetime can be oriented by $\nabla t$. With this choice of orientation, the future corresponds to the direction where $t$ increases (expanding direction) and the past to the direction where $t$ decreases (contracting direction).

${ }^{7}$ Note that, in the surface-symmetric case, a result analogous to Birkhoff's theorem applies, by which we mean that these spacetimes have no dynamical degree of freedom in the vacuum.

${ }^{8}$ Note that, in [Chruściel et al. 1990], strong cosmic censorship was also proved for polarized Gowdy spacetimes arising from initial data given on $S^{2} \times S^{1}, S^{3}$ and $L(p, q)$.
} 
the extra assumption that $t_{0}=0$. In [Smulevici 2008], we studied the remaining cases, namely the $T^{2}$ symmetric spacetimes with $\Lambda>0$ and Vlasov matter for which $t_{0}>0$, and proved their inextendibility, thus completing a proof of strong cosmic censorship for $T^{2}$-symmetric spacetimes with $\Lambda \geq 0$ and Vlasov matter. In the same article, we proved that vacuum $T^{2}$-symmetric spacetimes with $\Lambda>0$ and $t_{0}>0$ were also generically inextendible. Finally, in the surface-symmetric case with Vlasov matter, strong cosmic censorship was resolved in the affirmative for $k \leq 0$ and $\Lambda \geq 0$ and for $k=1$ and $\Lambda=0$, some obstructions remaining in the spherical case with $\Lambda>0$, in particular the possible formation of Schwarzschild-de-Sitter or, even worse, extremal Schwarzschild-de-Sitter black holes [Dafermos and Rendall 2007].

1C. The past asymptotic value of $t$ and the main theorems. The results of [Smulevici 2008], as well as the proof of inextendibility for the $k \leq 0$ surface-symmetric cases where $t_{0}>0$ contained in [Dafermos and Rendall 2007], gave satisfactory answers to the strong cosmic censorship conjecture. However, they did not address the question of the value of $t_{0}$. It is the subject of this article to resolve this question.

First, in Theorem 1 (see Section 5), we will extend the work of M. Weaver [2004] proving that the maximal Cauchy development of $T^{2}$-symmetric initial data with $\Lambda \geq 0$ and nonvanishing Vlasov matter can be covered by global areal foliations with $t$ going to zero in the contracting direction. Thus $t_{0}=0$ for these spacetimes.

As often happens in these types of problems, the vacuum case is more difficult than the Vlasov case. This is already reflected in the fact that for vacuum $T^{2}$-symmetric spacetimes with $\Lambda>0$, one can find special families of (nonflat) solutions for which $t_{0}>0$. That these solutions are indeed special is the content of Theorem 2 which states that vacuum $T^{2}$-symmetric spacetimes with $\Lambda \geq 0$ for which $t_{0}>0$ are necessarily polarized. Thus, generically, $t_{0}=0$ for vacuum $T^{2}$-symmetric spacetimes with $\Lambda \geq 0$.

Finally, we will show that the proof given for the $T^{2}$ case with Vlasov matter may be adapted to the hyperbolic case. We will obtain Theorem 3 which asserts that $t_{0}=0$ for $k \leq 0$ surface-symmetric spacetimes with $\Lambda \geq 0$ and nonvanishing Vlasov matter. Thus Theorem 3 asserts that the results of [Rein 1996; Tchapnda 2004] are true in general and do not require any smallness assumption. To summarize, we provide in Tables 1 and 2 a picture of the current status of the analysis of singularities for the $T^{2}$ symmetric and surface-symmetric spacetimes in the vacuum or with Vlasov matter.

1D. Outline. The outline of this article is as follows. We start in Section 2 with an introduction to the different classes of symmetry and present the classes of initial data that we will consider in the rest of the paper. In Section 3, we recall the existence and uniqueness of the maximal Cauchy development and in Section 4, we present the previous results concerning the global foliations of $T^{2}$-symmetric and $k \leq 0$ surface-symmetric spacetimes that we shall use as a starting point for our analysis. The statements of the theorems proved in this article then follow in Section 5. Before giving the proofs of the three theorems in sections 7,8 and 9, it will be useful to describe the approach that we will take, especially for the proof of Theorem 2, and this is done in Section 6. We end this paper with some comments and open questions in Section 10. In Appendix A, we provide some information on the initial data sets of the Einstein and Einstein-Vlasov systems for the reader not familiar with this. In Appendix B, we very briefly describe a coordinate transformation for $k=-1$ surface symmetric spacetimes and finally in Appendix C, we recall the classical results that symmetric initial data lead to symmetric spacetimes. 


\begin{tabular}{|c|c|c|c|c|}
\hline$T^{3}$-Gowdy & $\begin{array}{l}\text { vacuum } \\
\text { Vlasov }\end{array}$ & $\begin{array}{l}\Lambda=0 \\
\Lambda>0 \\
\Lambda=0 \\
\Lambda>0\end{array}$ & $\begin{aligned} t_{0} & =0 \\
t_{0} & =0 \\
t_{0} & =0 \\
t_{0} & =0\end{aligned}$ & $\begin{array}{l}\text { [Moncrief 1981; Chruściel 1990] } \\
\text { Theorem } 2 \\
\text { [Weaver 2004] } \\
\text { Theorem } 1\end{array}$ \\
\hline$T^{2}$-symmetric & $\begin{array}{l}\text { vacuum } \\
\text { Vlasov }\end{array}$ & $\begin{array}{l}\Lambda=0 \\
\Lambda>0 \\
\Lambda=0 \\
\Lambda>0\end{array}$ & $\begin{aligned} t_{0} & =0 \\
t_{0} & =0 \\
t_{0} & =0 \\
t_{0} & =0\end{aligned}$ & $\begin{array}{l}\text { [Isenberg and Weaver 2003] } \\
\text { Theorem } 2 \\
\text { [Weaver 2004] } \\
\text { Theorem } 1\end{array}$ \\
\hline$k=0$ & Vlasov & $\begin{array}{l}\Lambda=0 \\
\Lambda>0\end{array}$ & $\begin{array}{l}t_{0}=0 \\
t_{0}=0\end{array}$ & $\begin{array}{l}\text { [Weaver 2004] } \\
\text { Theorem 3; see also [Tchapnda 2004] with small data }\end{array}$ \\
\hline$k=-1$ & Vlasov & $\begin{array}{l}\Lambda=0 \\
\Lambda>0\end{array}$ & $\begin{array}{l}t_{0}=0 \\
t_{0}=0\end{array}$ & $\begin{array}{l}\text { Theorem 3; see also [Rein 1996] with small data } \\
\text { Theorem 3; see also [Tchapnda 2004] with small data }\end{array}$ \\
\hline
\end{tabular}

Table 1. Value of $t_{0}$ for generic $T^{2}$-symmetric and $k \leq 0$ surface-symmetric spacetimes.

\begin{tabular}{|c|c|l|}
\hline \multirow{2}{*}{$T^{3}$-Gowdy } & vacuum $\left\{\begin{array}{l}\Lambda=0 \\
\Lambda>0\end{array}\right.$ & Holds [Chruściel et al. 1990; Ringström 2006; 2009] \\
& Vlasov $\left\{\begin{array}{l}\Lambda=0 \\
\Lambda>0\end{array}\right.$ & Holds [Dafermos and Rendall 2006] \\
\hline \multirow{2}{*}{$T^{2}$-symmetric } & Holds [Dafermos and Rendall 2006; Smulevici 2008] \\
\hline$k=0$ & Vlasov $\left\{\begin{array}{l}\Lambda=0 \\
\Lambda>0\end{array}\right.$ & Open \\
$\Lambda>0$ & Holds for cases with $t_{0}>0$ [Smulevici 2008]; open otherwise & Holds [Dafermos and Rendall 2006] \\
\hline$k=1$ & Vlasov $\left\{\begin{array}{l}\Lambda=0 \\
\Lambda>0\end{array}\right.$ & Holds [Dafermos and Rendall 2007] \\
\hline \multirow{2}{*}{$k=0$} & Vlasov $\left\{\begin{array}{l}\Lambda=0 \\
\Lambda>0\end{array}\right.$ & Holds [Dafermos and Rendall 2007] \\
\hline
\end{tabular}

Table 2. Status of strong cosmic censorship for $T^{2}$-symmetric and surface-symmetric spacetimes.

\section{Preliminaries}

2A. $T^{2}$-symmetric spacetimes with spatial topology $\boldsymbol{T}^{\mathbf{3}}$. A spacetime $(\mathcal{M}, g)$ is said to be $T^{2}$-symmetric if the metric is invariant under an effective action of the Lie group $T^{2}$ and the group orbits are spatial. The Lie algebra of $T^{2}$ is spanned by two commuting Killing fields $X$ and $Y$ everywhere nonvanishing and we may normalize them so that the area element $t$ of the group orbits is given by

$$
g(X, X) g(Y, Y)-g(X, Y)^{2}=t^{2} .
$$

In previous analysis of these spacetimes [Chruściel 1990; Berger et al. 1997; Andréasson et al. 2004; Clausen and Isenberg 2007], it has been shown that any nonflat globally hyperbolic $T^{2}$-symmetric spacetime with spatial topology $T^{3}$ that satisfies the Einstein equations in the vacuum or with Vlasov matter 
and with $\Lambda \geq 0$ admits a metric in areal coordinates of the form

$$
d s^{2}=e^{2(\nu-U)}\left(-\alpha d t^{2}+d \theta^{2}\right)+e^{2 U}(d x+A d y+(G+A H) d \theta)^{2}+e^{-2 U} t^{2}(d y+H d \theta)^{2},
$$

where all functions depend only on $t$ and $\theta$ and are periodic in the latter. Note that the form (2) of the metric is unchanged under an $\operatorname{SL}(2, \mathbb{R})$ transformation of the Killing vectors $X=\partial / \partial x$ and $Y=\partial / \partial y$.

As $T^{2}$-symmetric spacetimes contain several dynamical degrees of freedom, certain special cases have been introduced. A first simplification appears in the case where the Killing fields $X$ and $Y$ may be chosen such that their inner product, and thus the function $A$, vanishes. Such cases are called polarized $T^{2}$-symmetric spacetimes.

Associated with $T^{2}$-symmetric spacetimes are certain quantities called the twist quantities, defined by

$$
\begin{gathered}
J=\epsilon_{a b c d} X^{a} Y^{b} \nabla^{c} X^{d}, \\
K=\epsilon_{a b c d} X^{a} Y^{b} \nabla^{c} Y^{d} .
\end{gathered}
$$

These are related to the metric functions by

$$
\begin{aligned}
& J=-\frac{t e^{-2 v+4 U}}{\sqrt{\alpha}}\left(G_{t}+A H_{t}\right), \\
& K=A J-\frac{t^{3} e^{-2 v}}{\sqrt{\alpha}} H_{t} .
\end{aligned}
$$

For any pair of commuting Killing vectors on a spacetime satisfying the vacuum Einstein equations, the associated twists quantities are constant [Geroch 1972]. Thus, for vacuum $T^{2}$-symmetric spacetimes, by an $\operatorname{SL}(2, \mathbb{R})$ transformation of the Killing fields $X$ and $Y$, we may ensure that the form of the metric (2) is unchanged while one of twist quantities vanishes. Therefore, in the vacuum, we shall always assume that $J=0$.

The cases where both $J=0$ and $K=0$ are called $T^{3}$-Gowdy spacetimes. By Frobenius's theorem, the conditions $J=K=0$ are equivalent to the integrability of the planes orthogonal to $d x, d y$.

2B. Spacetimes with a hyperbolic surface of symmetry. A spacetime $(\mathcal{M}, g)$ is said to be $k=-1$ surface-symmetric if it can be foliated by spacelike surfaces $\Sigma_{t}$ such that, for all $t, \Sigma_{t}$ is isometric to a doubly warped product $\left(S^{1} \times \mathscr{Y}, h_{t}\right)$ where $S$ is a fixed compact surface of constant curvature -1 .

It follows from previous analysis [Rendall 1995] that any $k=-1$ surface symmetric spacetime which is globally hyperbolic and satisfies the Einstein equations with $\Lambda \geq 0$, in the vacuum or with Vlasov matter, admits a metric in areal coordinates of the form ${ }^{9}$

$$
d s^{2}=-\frac{e^{2 v}}{t}\left(\alpha d t^{2}-d \theta^{2}\right)+t \gamma_{a b} d x^{a} d x^{b},
$$

where the functions $v$ and $\alpha$ depend only on $t$ and $\theta$ and are periodic in the latter with period 1 and $\gamma$ induces a metric of constant curvature -1 on the orbits of symmetry.

\footnotetext{
${ }^{9}$ Compared to the usual metric for these spacetimes, we use the square of the radius function $t=r^{2}$ as the time coordinate rather than the radius function $r$ itself. Moreover, we have introduced the functions $\alpha$ and $v$ by analogy with the $T^{2}$ case, so as to ease the application of the method of the $T^{2}$ case to this class of spacetimes. See Appendix B for a description of the change of coordinates from the usual parametrization to this one.
} 
2C. The Einstein-Vlasov system. Apart from the vacuum case, where we will set $T_{\mu \nu}=0$ in (1), we will couple the Einstein equations to the Vlasov matter model, which we present in this section.

Let $\mathscr{P} \subset \mathscr{T} M$ denote the set of all future-directed timelike vectors of length -1 . $\mathscr{P}$ is classically called the mass shell. Let $f$ denote a nonnegative function on the mass shell. The Vlasov equation for $f$ is derived from the condition that $f$ be preserved along geodesics. In coordinates, we therefore have

$$
p^{\alpha} \partial_{x^{\alpha}} f-\Gamma_{\beta \gamma}^{\alpha} p^{\beta} p^{\gamma} \partial_{p^{\alpha}} f=0,
$$

where $p^{\alpha}$ denotes the momentum coordinates on the tangent bundle conjugate to $x^{\alpha}$.

The energy-momentum tensor is defined by

$$
T_{\alpha \beta}(x)=\int_{\pi^{-1}(x)} p_{\alpha} p_{\beta} f,
$$

where $\pi: \mathscr{P} \rightarrow \mathcal{M}$ is the natural projection from the mass shell to the spacetime and the integral is with respect to the natural volume form on $\pi^{-1}(x)$.

2D. The classes of initial data. After this introduction to the symmetry classes and the matter fields, we are ready to present the initial data sets that will be studied in this article. For convenience, we will require that the initial data be smooth and, in the nonvacuum case, that the support of the Vlasov field be compact. These assumptions may clearly be relaxed if necessary. ${ }^{10}$

Definition 1. A vacuum $T^{2}$-symmetric initial data set is a triplet $(\Sigma, h, K)$ such that

(1) $\Sigma$ is a smooth differential 3-manifold with topology $T^{3}$ (in particular, $\Sigma$ admits an effective action of $T^{2}$ ),

(2) $h$ is a smooth Riemannian metric on $\Sigma$ that is invariant under an effective action of the Lie group $T^{2}$,

(3) $K$ is a smooth symmetric 2-tensor also invariant under the same $T^{2}$ action,

(4) $\left(\Sigma, h, K_{a b}\right)$ satisfies the vacuum constraint equations of general relativity.

We describe in Appendix A the constraint equations in the vacuum or in the presence of Vlasov matter for the reader not familiar with them.

Definition 2. A $T^{2}$-symmetric initial data set with Vlasov matter is a quadruplet $(\Sigma, h, K, \hat{f})$ such that

(1) conditions (1), (2) and (3) of the preceding definition hold,

(2) $\hat{f}$ is a smooth, nonnegative function of compact support defined on $T \Sigma$ which is invariant under the natural lift to $T \Sigma$ of the $T^{2}$ action,

(3) $\left(\Sigma, h, K_{a b}, \hat{f}\right)$ satisfies the constraint equations of the Einstein-Vlasov system.

Let us also define the notion of polarized $T^{2}$-symmetric initial data and of Gowdy initial data:

Definition 3. A vacuum $T^{2}$-symmetric initial data set $(\Sigma, h, K)$ (respectively a $T^{2}$-symmetric initial data set with Vlasov matter $(\Sigma, h, K, \hat{f}))$ is said to be polarized if there exist two Killing fields $(X, Y)$ which generate the $T^{2}$ action such that $h(X, Y)=0$ and $K(X, Y)=0$ on $\Sigma$.

\footnotetext{
${ }^{10}$ For instance, we could have chosen the initial data to be compatible with the statement of Theorem 4.1 of [Dafermos and Rendall 2006]. However, we decided to give preference to clarity and will therefore stick with compact data for the Vlasov field.
} 
Definition 4. A vacuum $T^{2}$-symmetric initial data set $(\Sigma, h, K)$ (respectively a $T^{2}$-symmetric initial data set with Vlasov matter) is said to be a Gowdy initial data set if there exist linearly independent, commuting vector fields $Z, X, Y$ on $\Sigma$ such that $X, Y$ are Killing fields which generate the $T^{2}$ action and such that $h(Z, X)=h(Z, Y)=K(Z, Y)=K(Z, X)=0$.

We define $k=-1$ surface-symmetric initial data with Vlasov matter as follows:

Definition 5. A $k=-1$ surface-symmetric initial data set with Vlasov matter is a quadruplet $(\Sigma, h, K, \hat{f})$ such that

(1) $\Sigma=S^{1} \times \mathscr{Y}$ where $\mathscr{Y}$ is a smooth compact surface,

(2) $h$ is a smooth Riemannian doubly-warped product metric on $\Sigma$ of the form $a(\theta) d \theta^{2}+b(\theta) \gamma_{\mathscr{S}}$, where $\gamma_{9}$ is a metric of constant curvature -1 ,

(3) $\hat{f}$ is a smooth, nonnegative function of compact support defined on $T \Sigma$ and invariant under the natural lift of the local isometries of $\mathscr{Y}$ to $T \Sigma$,

(4) $\left(\Sigma, h, K_{a b}, \hat{f}\right)$ satisfies the constraint equations of the Einstein-Vlasov system.

\section{The maximal Cauchy development}

We will recall in this section the classical results concerning the existence and uniqueness of the maximal Cauchy development to which we will refer often in the rest of this article. We will state the theorem in the case of Vlasov matter. For the vacuum case, it suffices to replace all matter terms by zero.

Theorem. Let $(\Sigma, h, K, \hat{f})$ be an initial data set for the Einstein-Vlasov system. There exists a triplet $(\mathcal{M}, g, f)$, called the maximal Cauchy development of $(\Sigma, h, K, \hat{f})$, satisfying these conditions:

(1) $(M, g)$ is a smooth globally hyperbolic spacetime and $f$ is a smooth, nonnegative function of compact support defined on the mass shell $\mathscr{P}$.

(2) $(M, g, f)$ satisfies the Einstein-Vlasov system (1), (9), (8).

(3) There exists a smooth embedding $\phi: \Sigma \rightarrow M$ such that $\phi(\Sigma)$ is a Cauchy surface for $M$ and if $h^{\prime}$, $K^{\prime}, f^{\prime}$ denotes respectively the first and second fundamental form of $\phi(\Sigma)$ and the restriction of $f$ to the tangent bundle of $\phi(\Sigma)$ then $\phi^{*}\left(h^{\prime}\right)=h, \phi^{*}\left(K^{\prime}\right)=K, \phi^{*}\left(f^{\prime}\right)=\hat{f}$.

(4) If $(\bar{M}, \bar{g}, \bar{f})$ is another triplet satisfying (1), (2) and (3) and if $\bar{\phi}$ denotes the corresponding embedding of $\Sigma$ in $\bar{M}$ then there exists an smooth isometry $\psi$ from $(\bar{M}, \bar{g})$ onto a subset of $(\mu, g)$ such that $\psi^{*} \bar{f}=f$ and $\psi(\bar{\phi}(\Sigma))=\phi(\Sigma)$.

See [Fourès-Bruhat 1952; Choquet-Bruhat and Geroch 1969; Choquet-Bruhat 1970; 1971] for the original proofs of these results.

\section{Global areal foliations of $T^{2}$-symmetric or $k=-1$ surface-symmetric spacetimes}

We present in this section certain previous results concerning areal foliations of $T^{2}$-symmetric or $k=-1$ surface-symmetric spacetimes. Let us first recall that symmetries of the initial data are transmitted to the maximal Cauchy development. For the reader not familiar with these results, they are presented in Appendix C. Thus, $T^{2}$-symmetric (respectively surface-symmetric) initial data lead to $T^{2}$-symmetric (respectively surface-symmetric) spacetimes. 
From [Chruściel 1990; Berger et al. 1997; Andréasson et al. 2004; Clausen and Isenberg 2007] for the $T^{2}$ case and from [Andréasson et al. 2003] for the hyperbolic case, we have:

Proposition 1. Let $(M, g, f)$ be the maximal Cauchy development of nonflat $T^{2}$-symmetric initial data (respectively $k=-1$ surface-symmetric initial data) with $\Lambda \geq 0$, either in the vacuum or with Vlasov matter.

(1) $(M, g)$ is $T^{2}$-symmetric (respectively $k=-1$ surface-symmetric) and $f$ is invariant under the natural lift to $T M$ of the $T^{2}$ action (respectively under the natural lift to $T M$ of the local isometries of $\mathscr{Y}$, with $\mathscr{S}$ as in Definition 5).

(2) $(M, g)$ can be covered by areal coordinates $(t, \theta, x, y)$ where the metric takes the form (2) (respectively (7)) and $t$ ranges from $t_{0} \geq 0$ to $+\infty$.

(3) In the $T^{2}$ case, $(M, g)$ is a polarized $T^{2}$-symmetric spacetime (respectively a $T^{3}$-Gowdy spacetime) if and only if the initial data are polarized (respectively Gowdy).

We also have a continuation criterion, which follows from the standard local well-posedness theory for the Einstein-Vlasov system as found in [Choquet-Bruhat 1970; 1971]:

Proposition 2. Let $(\mathcal{M}, g, f)$ be a past development ${ }^{11}$ of $T^{2}$-symmetric initial data (respectively $k=-1$ surface-symmetric initial data) with $\Lambda \geq 0$, either in the vacuum or with Vlasov matter and assume that $(M, g)$ can be covered by areal coordinates $(t, \theta, x, y)$, where $t$ ranges from $t_{f}>0$ to $t_{i}, t_{f}<t_{i}$ and the metric takes the form (2) (respectively (7)). Assume that

(1) all metric functions and their derivatives admit a continuous extension to $t=t_{f}$, and

(2) in the Vlasov case, $f$ and all its derivatives admit a continuous extension to $t=t_{f}$.

Then there exists a past development $(\tilde{M}, \tilde{g}, \tilde{f})$ of the initial data and an isometric embedding $i$ of $M$ into $\tilde{M}$ satisfying $i^{*}(\tilde{f})=f$ and such that $i(\mathcal{M}) \neq \tilde{M}$.

\section{The theorems}

Theorem 1. Let $(\mathcal{M}, g, f)$ be the maximal development of $T^{2}$-symmetric initial data with Vlasov matter and $\Lambda \geq 0$. Suppose that the Vlasov field $f$ does not vanish identically. Then $(\mathcal{M}, g)$ admits a global foliation by areal coordinates with the time coordinate taking all values in $(0, \infty)$; that is, $t_{0}=0$ in the notation of Proposition 1.

Thus the presence of Vlasov matter forbids $t_{0}>0$. In the vacuum case, we know that nonflat solutions with $t_{0}>0$ exist in [Smulevici 2008, Appendix E], an indication that this case is more difficult.

Theorem 2. Let $(M, g)$ be the maximal Cauchy development of vacuum $T^{2}$-symmetric initial data with $\Lambda>0$ and suppose that the spacetime is not polarized. Then $(M, g)$ admits a global foliation by areal coordinates with the time coordinate t taking all values in $(0, \infty)$; that is, $t_{0}=0$ in the notation of Proposition 1.

The last theorem is the analogous of Theorem 1 in the hyperbolic symmetric case:

\footnotetext{
${ }^{11}$ Here and everywhere else in the paper, we will consider that, by definition, a development of an initial data set for the Einstein equations is a globally hyperbolic spacetime that satisfies the Einstein equations and agrees with the given data initially in the usual sense of general relativity.
} 
Theorem 3. Let $(M, g, f)$ be the maximal development of $k=-1$ surface-symmetric initial data with Vlasov matter and $\Lambda \geq 0$. Suppose that the Vlasov field $f$ does not vanish identically. Then $(M, g)$ admits a global foliation by areal coordinates with the time coordinate t taking all values in $(0, \infty)$; that is, $t_{0}=0$ in the notation of Proposition 1.

Note that in the vacuum case, there exist solutions of the Einstein equations with hyperbolic symmetry such that $t_{0}>0$ [Rendall 1995]. Thus, the assumption on the Vlasov field is necessary.

\section{Remarks on the strategy of the proofs}

We will present here the main ideas of the proofs of the theorems. We will place particular emphasis on the proof of Theorem 2 as it is the most difficult one. The reader might want to return to this section while reading the proof of Theorem 2 in order to better follow the arguments.

The proofs of Theorems 1 and 3 are based on the strategies developed in [Isenberg and Weaver 2003; Weaver 2004]. However, some crucial arguments of these previous works fail in the case of Theorem 2 and we have thus been forced to introduce a different approach, which we will present below.

In order to explain these differences and before presenting this new approach, let us first briefly revisit some of the ideas of the proofs contained in [Isenberg and Weaver 2003] and [Weaver 2004] for $T^{2}$ symmetric spacetimes with $\Lambda=0$, respectively in the vacuum and in the Vlasov case.

6A. Previous work. Let us thus assume that $(M, g, f)$ is a past development of $T^{2}$-symmetric initial data, with $\Lambda=0$, in the vacuum or with Vlasov matter. Suppose that $(\mathcal{M}, g)$ is covered by areal coordinates with $t \in\left(t_{f}, t_{i}\right]$, where $t_{f}>0$. In view of Proposition 2, in order to obtain a statement analogue to that of Theorem 1 , it is sufficient to prove that for all such $(\mathcal{M}, g)$, all metric functions, the Vlasov field $f$ and all their derivatives admit continuous extensions to $t=t_{f}$.

The conformal coordinate system and the function $\alpha$. Let us first recall from [Berger et al. 1997] that another coordinate system may be introduced in $(\mathcal{M}, g)$, the so-called conformal coordinate system. In this coordinate system, the metric takes the form

$$
d s^{2}=e^{2(\nu-U)}\left(-d \tau^{2}+d \chi^{2}\right)+e^{2 U}(d x+A d y+(G+A H) d \chi)^{2}+e^{-2 U} t^{2}(d y+H d \chi)^{2} .
$$

In the coordinate system $(\tau, \chi, x, y)$, if one assumes that the area of the symmetry orbits $t$ is uniformly bounded from below by a strictly positive constant, one may obtain ${ }^{12}$ continuous extensions of all metric functions, the Vlasov field and their derivatives [Berger et al. 1997]. Thus, it is clear that in order to obtain the same statement in areal coordinates, the key point is to control the function $\alpha$ appearing in (2), as well as its derivatives, since it is this function which dictates the change of coordinates from conformal to areal coordinates. Moreover, it turns out that the function $\alpha$ is necessarily nondecreasing in the past, and in fact increasing if $K>0$ (i.e., the spacetime is not of $T^{3}$-Gowdy type). This implies that, in essence, one only need prove that $\alpha$ is bounded above.

\footnotetext{
${ }^{12}$ The proof (in the vacuum case) is essentially based on energy and null cone estimates where the energies considered arose naturally from the wave map background structure of the equations. On the other hand, these estimates and the results obtained in conformal coordinates do not provide any information concerning the behavior of the function $t$, apart from what is already contained in the statement of Proposition 1, see [Berger et al. 1997].
} 
The energy estimates. For this purpose, one introduces the energy density ${ }^{13}$

$$
g=U_{t}^{2}+\alpha U_{\theta}^{2}+\frac{e^{2 U}}{4 t^{2}}\left(A_{t}^{2}+\alpha A_{\theta}^{2}\right)
$$

and the energy integral

$$
E_{g}=\int_{\theta \in[0,1]} \frac{g}{\sqrt{\alpha}} .
$$

This energy can easily be shown to be bounded from above.

The estimate on $\alpha$. Moreover, one can obtain an estimate of the type:

$$
\alpha e^{2 v}(t, \theta) \leq C \frac{E_{g}\left(t_{i}\right)}{E_{g}(t)},
$$

for some positive constant $C$ which depends only on the initial data and the value of $t_{f}>0$. Thus, in order to obtain an upper bound on $\alpha e^{2 v}$, it is sufficient to have a lower bound on $E_{g}$. In the vacuum case with $\Lambda=0$, this lower bound follows easily from the Einstein equations, as $E_{g}$ is necessarily nondecreasing in the past direction. From the bound on $\alpha e^{2 v}$, the upper bound on $\alpha$ follows easily by integration of the evolution equation for $\alpha$; see (106) with $\Lambda=0$. The key points are thus the estimate (13) and the monotonicity of $E_{g}$.

In the Vlasov case, the monotonicity of $E_{g}$ is actually broken and thus one loses the easy upper bound on $\alpha e^{2 v}$. In order to obtain a bound on $\alpha$, one introduces another energy integral, which we shall call here $E_{g, f}$, which can also be proven to be bounded from above. It turns out that $E_{g, f}$ controls $\rho$, an energy density associated with the energy-momentum tensor, and using the fact that $f$ does not vanish identically, Weaver [2004] proved that one can extract enough information from $\rho$ to obtain the estimate

$$
\min _{\theta \in[0,1]} \alpha(t, \cdot)<M,
$$

for some constant $M>0$. Thus, using the fact that $f$ does not vanish, one obtains an estimate on the function $\alpha$. This estimate is not as strong as in the vacuum case but it turns out that, together with the upper bound on $E_{g}$, this control on $\alpha$ is sufficient to derive pointwise estimates on $g$ and bounds on the support of $f$, from which it is easy to derive all the remaining estimates.

6B. The proofs of Theorem 1 and Theorem 3. Assume now that we are in the setting of Theorem 1, where we focus on the $T^{2}$-symmetric case with Vlasov matter and $\Lambda \geq 0$. In this case, as in the case where $\Lambda=0, f \neq 0$ discussed in Section 6A, we do not have monotonicity of $E_{g}$. However, all other important monotonicity properties hold and the estimate concerning $\min _{\theta \in[0,1]} \alpha(t, \cdot)$ still holds. This implies that the proof in the Vlasov case with $\Lambda=0$ can be extended without too much difficulty to the case where $\Lambda>0$. This is treated in detail in Section 7 .

Remark 6B.1. In particular, we note that the assumption of the nonvanishing of the Vlasov field is necessary only so as to establish the estimate (14). In other words, we have the following proposition, which will be useful in the course of the proof of Theorem 2.

\footnotetext{
${ }^{13}$ In the Gowdy case, this energy quantity arises naturally from the wave map structure of the equations. For the $T^{2}$ case, the vacuum Einstein equations may be regarded as the equations of a wave map problem with source, for which the natural associated energy density is $g$.
} 
Proposition 3. Let $(\mathcal{M}, g, f)$ be a development of $T^{2}$-symmetric initial data in the vacuum or with Vlasov matter and with $\Lambda \geq 0$. Assume that $(\mathcal{M}, g)$ admits a global areal foliation $(t, \theta, x, y)$ where $t$ ranges from $t_{f}>0$ to $t_{i}, t_{f}<t_{i}$. Assume moreover that the estimate (14) holds. Then, all the metric functions, the Vlasov field $f$ and all their derivatives admits continuous extensions to $t=t_{f}$, i.e the assumptions of Proposition 2 are verified.

Let us also note that the important monotonicity properties used in the proof of Theorem 1 remain valid in the case of hyperbolic symmetry. We will prove Theorem 3 by adapting the strategy of the proof of Theorem 1 to the hyperbolic symmetric case. This is treated in detail in Section 9.

6C. The proof of Theorem 2. In the vacuum case with $\Lambda>0$, we lose again the monotonicity property of $E_{g}$. Thus, one does not have a priori the lower bound on $E_{g}$ required to apply (13). Moreover, we cannot obtain an a priori estimate on $\min _{\theta \in[0,1]} \alpha(t, \cdot)$ as in the Vlasov case as this required that certain matter terms do not vanish. However, estimates similar to (13) hold and thus, we easily obtain that the statement that $\alpha$ is bounded above is equivalent to the statement that $E_{g}$ is bounded from below by a strictly positive constant.

Different parametrizations for the orbits of symmetry and explicit solutions of the equations. The monotonicity of $E_{g}$ is linked with the homogeneity or inhomogeneity of the wave equation for the metric function $U$ defined in (2). When $\Lambda>0$, an extra term arises in the time derivative of $E_{g}$ which has the wrong sign; see (124). In fact, when both twists quantities vanish, i.e., in the $T^{3}$-Gowdy case $(K=0)$, there is a way to recover the monotonicity argument. Indeed, one may apply a simple transformation to the function $U$ such that the wave equation for the resulting metric function $P$ is homogeneous; see (113) with $K=0$. Using $(U, A)$ or $(P, A)$ corresponds to a different choice of parametrization for the extrinsic geometry of the orbits of symmetry. The system of wave equations satisfied by $(P, A)$ has a similar structure to that of $(U, A)$ and one may introduce an energy $E_{h}$ associated with it, which plays a role similar to that of $E_{g}$.

The interpretation of the transformation is as follows. In the case $(K=0, \Lambda=0)$, all flat Kasner spacetimes corresponding to $U=k \ln t, A=$ constant are possible solutions of the equations. In the case ( $K>0, \Lambda=0$ ), the only Kasner spacetimes of the form $U=k \ln t, A=$ constant satisfying the Einstein equations are those for which $U=0$ and $A=$ constant. Another characterization of these solutions is that they correspond to $E_{g}=0$. In the case $K=0, \Lambda>0$, there can be naturally no flat Kasner solutions, but there are plane symmetric solutions which are characterized by $E_{h}=0$. We also remark that in both cases, $(K>0, \Lambda=0)$ and $(K=0, \Lambda>0)$, there are solutions, with respectively $E_{g}=0$ or $E_{h}=0$, for which $t_{0}>0$; see [Isenberg and Weaver 2003; Smulevici 2008, Appendix E].

The easy case $(K=0, \Lambda>0)$. In this case, as mentioned above, the system of wave equations for $(P, A)$ is homogeneous. Moreover, one can easily prove that $E_{h}$ is nondecreasing in the past direction (see Remark 8E.1). An estimate similar to (13) can be derived, from which we obtain the desired upper bound on $\alpha$ under the assumption that $E_{h} \neq 0$ initially. This case can thus be treated separately and we present it in Proposition 4 (see Section 8E).

The general case. The contradiction setting. When both $K>0$ and $\Lambda>0$, there is no easy way to recover a monotonicity property on $E_{h}$ or $E_{g}$ and thus there are no a priori lower bounds on $E_{g}$ or $E_{h}$. We will prove Theorem 2 by recovering such a lower bound via other methods. The aim will therefore 
be to bound away from 0 the energy integrals $E_{h}$ and $E_{g}$ associated with the nonlinear system of wave equations describing the motion of the orbits of symmetry. For this, we will proceed by contradiction, assuming that $t_{0}>0$ for the maximal Cauchy development.

This will allow us to obtain two important facts: $\alpha$ is uniformly blowing up (Section $8 \mathrm{C}$ ) and the energy integrals $E_{g}$ and $E_{h}$ tend to 0 as $t \rightarrow t_{0}$ (Section $8 \mathrm{H}$ ). (The uniform blow up of $\alpha$ is in fact an immediate consequence of the Remark 6B.1.)

Control on the spatial differences of some metric functions. We will use the uniform blow up of $\alpha$ and the vanishing limit of $E_{h}$ and $E_{g}$ to obtain successively more and more control on the solutions and improve our understanding of the nonlinear terms in the equations. First, the vanishing of $E_{h}$ and $E_{g}$ in the limit $t \rightarrow t_{0}$ will imply a strong control on the spatial differences of some the metric functions (Section 8I). In particular, control on $\max _{\theta \in[0,1]} \alpha e^{v}-\min _{\theta \in[0,1]} \alpha e^{v}$ and similar quantities will be used extensively in the null cone estimates and the analysis of the characteristics which we will pursue later.

Some tools for the null cone estimates. In Sections $8 \mathrm{O}$ and 8P, we will derive null cone estimates. In order to do so, it will be necessary to have at hand the following tools:

- an estimate on $(\partial / \partial \theta)(\ln \alpha)($ Section $8 \mathrm{~J})$,

- estimates for the integrals of small powers of $\alpha$ (Section $8 \mathrm{M}$ ), which will essentially be used to control some error terms in the null cone estimates,

- a parametrization of the null rays in areal coordinates (Section $8 \mathrm{~K}$ ).

Moreover, to exploit these null cone estimates in the last step of the proof, we will need to control a change of coordinates from the coordinates adapted to the null rays to the areal system of coordinates. The required estimate is proved in Section $8 \mathrm{~L}$.

Finally, in order to prove the pointwise estimates from below of Section 8P, we will need to start with large data. The analysis of the polarization energy, which we describe below, will enable us to exhibit such large data.

The polarization energy $E_{A}$. In Section $8 \mathrm{~L}$, we will focus our attention on the polarization energy $E_{A}$ of the spacetime associated with the wave equation satisfied by the polarization function $A$ defined in (2). Since by definition, $E_{A} \leq E_{h}$, a lower bound on $E_{A}$ is sufficient to obtain a lower bound on $E_{h}$ and close the estimates. (Motivation for considering this energy comes from the fact that the evolution equation for $A$ stays homogeneous even with $\Lambda>0$ and the simple remark that one of the common features of all known cases with $t_{0}>0$ is that all such spacetimes are polarized and thus have $E_{A}=0$.) From the contradiction setting, it follows that $E_{A} \rightarrow 0$ as $t \rightarrow t_{0}$. Using the assumption $E_{A}>0$ and the vanishing limit of $E_{A}$, we will exhibit a sequence of points in the spacetime where the energy density $h$ is of the order of $\alpha$.

The null cone estimates. These points will be used in Section 8P as large initial data for some null cone estimates along the characterisitics of the spacetime. The aim of these null cone estimates will be to prove that not only is $h$ of order $\alpha$ at some points, but it is in fact blowing up at least like $\alpha^{1-\epsilon}$ along certain characteristics. However, in order to control the spatial derivatives and the nonlinearity of the equations, we will also need an estimate from above for $h$. Thus, we will first prove that $h$ is blowing up at most like $\alpha^{1+\epsilon}$. To derive these pointwise estimates on $h$, we will use the tools developed in the previous sections and apply null cone estimates similar to those we introduced in [Smulevici 2008]. 
By a continuity argument, it will actually follow that $h$ necessarily blows up along a whole family of characteristics.

The contradiction. In the previous step, we have obtained the blow up of $h$ as $\alpha^{1-\epsilon}$ along a strip of characteristics. This can be integrated in space but if we want to relate the resulting integral to $E_{h}$, we need to control the difference between the integral of $h$ over the spacelike foliation associated with the conformal coordinate system and its integral over the spacelike foliations associated with the areal coordinate system. Using the results of Section $8 \mathrm{~L}$, we will prove in Section $8 \mathrm{Q}$ that the two integrals differ at most by a factor of $\alpha^{\epsilon}$. It follows that

$$
E_{h}=\int_{[0,1]} \frac{h}{\sqrt{\alpha}} d \theta
$$

is bounded from below by $\delta \min _{\theta \in[0,1]} \alpha^{1 / 2-2 \epsilon}$ for some $\delta>0$ and thus, in particular, does not vanish as $t$ goes to $t_{0}$. This is a contradiction, which concludes the proof of Theorem 2 .

\section{Proof of Theorem 1}

We will prove Theorem 1 in this section. As discussed above, the method will follow [Weaver 2004]. It would be sufficient to check that the extra terms arising from the introduction of $\Lambda>0$ do not spoil any of the monotonicity arguments and may be controlled when required, but in order to be self-contained, we will provide a full proof. Moreover, some of the estimates given here will be useful later in order to prove Theorem 2 in Section 8, in particular, to obtain the uniform blow up of $\alpha$ of Lemma 8.1. We start by recalling the Einstein-Vlasov system for $T^{2}$-symmetric spacetimes in areal coordinates.

7A. Vlasov matter in $\boldsymbol{T}^{\mathbf{2}}$-symmetric spacetimes. Let $(\mathcal{M}, g, f)$ be a past development of $T^{2}$-symmetric initial data with Vlasov matter as described in Section 2D and assume that $(t, \theta, x, y)$ is a system of areal coordinates such that the metric takes the form (2). Let $v_{i}$, for $i=0,1,2,3$, denote the components of the velocity vector in the untwisted set of 1 -forms:

$$
\{d t, d \theta, d x+G d \theta, d y+H d \theta\} .
$$

The dual basis is

$$
\left\{\frac{\partial}{\partial t}, \frac{\partial}{\partial \theta}-G \frac{\partial}{\partial x}-H \frac{\partial}{\partial y}, \frac{\partial}{\partial x}, \frac{\partial}{\partial y}\right\}
$$

In this new frame, the metric (2) and its inverse are given by

$$
\begin{aligned}
& \tilde{g}_{i j}=\left(\begin{array}{cccc}
-\alpha e^{2(\nu-U)} & 0 & 0 & 0 \\
0 & e^{2(\nu-U)} & 0 & 0 \\
0 & 0 & e^{2 U} & e^{2 U} A \\
0 & 0 & e^{2 U} A & e^{-2 U} t^{2}+e^{2 U} A^{2}
\end{array}\right), \\
& \tilde{g}^{i j}=\left(\begin{array}{cccc}
-\alpha^{-1} e^{-2(\nu-U)} & 0 & 0 & 0 \\
0 & e^{-2(\nu-U)} & 0 & 0 \\
0 & 0 & e^{-2 U}+e^{2 U} A^{2} t^{-2} & -e^{2 U} A t^{-2} \\
0 & 0 & -e^{2 U} A t^{-2} & e^{2 U} t^{-2}
\end{array}\right) .
\end{aligned}
$$


Note that, along a geodesic, the components $v_{2}$ and $v_{3}$ of the velocity vector are constant, since if we denote the tangent vector to a geodesic by $V$, the geodesic and the Killing equations give

$$
\begin{aligned}
v_{2} & =g\left(V, \frac{\partial}{\partial x}\right), \\
\nabla_{V} g\left(V, \frac{\partial}{\partial x}\right) & =g\left(\nabla_{V} V, \frac{\partial}{\partial x}\right)+g\left(V, \nabla_{V} \frac{\partial}{\partial x}\right)=0 .
\end{aligned}
$$

We will parametrize the mass shell $\mathscr{P}$ by the coordinates $\left(t, \theta, x, y, v_{1}, v_{2}, v_{3}\right)$, where by an abuse of notation we denote the lift to $\mathscr{P}$ of the coordinates on $\mathcal{M}$ by the same symbols. The Vlasov field $f$ can then be identified with a function of $\left(t, \theta, x, y, v_{1}, v_{2}, v_{3}\right)$ or, using the symmetry, with a function of $\left(t, \theta, v_{1}, v_{2}, v_{3}\right)$ only. We shall, abusing notation, use both definitions and always denote it by $f$.

With these definitions, the mass shell relation $v_{\mu} v^{\mu}=-1$, which holds on the support of the Vlasov field, is given by ${ }^{14}$

$$
v_{0}=-\sqrt{\alpha e^{2(v-U)}+\alpha v_{1}^{2}+\alpha e^{2(\nu-2 U)} v_{2}^{2}+\alpha t^{-2} e^{2 v}\left(v_{3}-A v_{2}\right)^{2}},
$$

and the Vlasov equation reads as

$$
\frac{\partial f}{\partial t}=\frac{\partial v_{0}}{\partial v_{1}} \frac{\partial f}{\partial \theta}-\left(\frac{\partial v_{0}}{\partial \theta}+\frac{\sqrt{\alpha} e^{v}}{t^{3}}(K-A J)\left(v_{3}-A v_{2}\right)+\frac{\sqrt{\alpha} e^{2 v-4 U}}{t} J v_{2}\right) \frac{\partial f}{\partial v_{1}}
$$

7B. The Einstein equations in areal coordinates. The Einstein equations (1) give rise to the following system of equations in areal coordinates:

\section{Constraint equations:}

$$
\begin{aligned}
\frac{v_{t}}{t}=U_{t}^{2}+\alpha U_{\theta}^{2}+\frac{e^{4 U}}{4 t^{2}}\left(A_{t}^{2}+\alpha A_{\theta}^{2}\right)+\frac{\alpha e^{2 \nu-4 U}}{4 t^{2}} J^{2} & +\frac{\alpha e^{2 v}(K-A J)^{2}}{4 t^{4}} \\
& +\alpha e^{2(\nu-U)} \Lambda+8 \pi \frac{\sqrt{\alpha}}{t} \int_{\mathbb{R}^{3}} f\left|v_{0}\right| d v_{1} d v_{2} d v_{3},
\end{aligned}
$$

$$
\begin{aligned}
\frac{\alpha_{t}}{\alpha}=-\frac{\alpha e^{2 v-4 U} J^{2}}{t}- & \frac{\alpha e^{2 v}(K-A J)^{2}}{t^{3}}-4 t \alpha e^{2(v-U)} \Lambda \\
& -16 \pi \alpha^{3 / 2} e^{2(v-U)} \int_{\mathbb{R}^{3}} \frac{f\left(1+e^{-2 U} v_{2}^{2}+e^{2 U} t^{-2}\left(v_{3}-A v_{2}\right)^{2}\right)}{\left|v_{0}\right|} d v_{1} d v_{2} d v_{3},
\end{aligned}
$$

$$
\frac{v_{\theta}}{t}=2 U_{t} U_{\theta}+\frac{e^{4 U}}{2 t^{2}} A_{t} A_{\theta}-\frac{\alpha_{\theta}}{2 t \alpha}-8 \pi \frac{\sqrt{\alpha}}{t} \int_{\mathbb{R}^{3}} f v_{1} d v_{1} d v_{2} d v_{3} \text {. }
$$

Evolution equations:

$$
\begin{aligned}
v_{t t}- & \alpha v_{\theta \theta}=\frac{\alpha_{\theta} v_{\theta}}{2}+\frac{\alpha_{t} v_{t}}{2 \alpha}-\frac{\alpha_{\theta}^{2}}{4 \alpha}+\frac{\alpha_{\theta \theta}}{2}-U_{t}^{2}+\alpha U_{\theta}^{2}+\frac{e^{4 U}}{4 t^{2}}\left(A_{t}^{2}-\alpha A_{\theta}^{2}\right) \\
& -\frac{\alpha e^{2 v-4 U} J^{2}}{4 t^{2}}-\frac{3 \alpha e^{2 v}(K-A J)^{2}}{4 t^{4}}+\alpha \Lambda e^{2(v-U)}-8 \pi \frac{\alpha^{3 / 2} e^{2 v}}{t^{3}} \int_{\mathbb{R}^{3}} \frac{f\left(v_{3}-A v_{2}\right)^{2}}{\left|v_{0}\right|} d v_{1} d v_{2} d v_{3},
\end{aligned}
$$

\footnotetext{
${ }^{14}$ Note that $v_{0}<0$ since $v^{0}>0$.
} 


$$
\begin{aligned}
U_{t t}-\alpha U_{\theta \theta}=- & \frac{U_{t}}{t}+\frac{\alpha_{\theta} U_{\theta}}{2}+\frac{\alpha_{t} U_{t}}{2 \alpha}+\frac{e^{4 U}}{2 t^{2}}\left(A_{t}^{2}-\alpha A_{\theta}^{2}\right) \\
& +\alpha \Lambda e^{2(\nu-U)}+\frac{\alpha e^{2 \nu-4 U} J^{2}}{2 t^{2}}+8 \pi \frac{\alpha^{3 / 2} e^{2(\nu-U)}}{2 t} \int_{\mathbb{R}^{3}} \frac{f\left(1+2 e^{-2 U} v_{2}^{2}\right)}{\left|v_{0}\right|} d v_{1} d v_{2} d v_{3},
\end{aligned}
$$

$$
\begin{aligned}
A_{t t}-\alpha A_{\theta \theta}=\frac{A_{t}}{t}+\frac{\alpha_{\theta} A_{\theta}}{2}+\frac{\alpha_{t} A_{t}}{2 \alpha}-4\left(A_{t} U_{t}-\alpha A_{\theta} U_{\theta}\right) & \\
& +\frac{\alpha e^{2 v-4 U} J(K-A J)}{t^{2}}+16 \pi \frac{\alpha^{3 / 2} e^{2 v-4 U}}{t} \int_{\mathbb{R}^{3}} \frac{f v_{2}\left(v_{3}-A v_{2}\right)}{\left|v_{0}\right|} d v_{1} d v_{2} d v_{3} .
\end{aligned}
$$

Auxiliary equations:

$$
\begin{aligned}
J_{t} & =-16 \pi \alpha \int_{\mathbb{R}^{3}} \frac{f v_{1} v_{2}}{\left|v_{0}\right|} d v_{1} d v_{2} d v_{3}, \\
J_{\theta} & =16 \pi \int_{\mathbb{R}^{3}} f v_{2} d v_{1} d v_{2} d v_{3}, \\
K_{t} & =-16 \pi \alpha \int_{\mathbb{R}^{3}} \frac{f v_{1} v_{3}}{\left|v_{0}\right|} d v_{1} d v_{2} d v_{3}, \\
K_{\theta} & =16 \pi \int_{\mathbb{R}^{3}} f v_{3} d v_{1} d v_{2} d v_{3} .
\end{aligned}
$$

We will now proceed to the proof of Theorem 1.

In the rest of this section, $(M, g, f)$ will be a past development of $T^{2}$-symmetric initial data with Vlasov matter and $\Lambda \geq 0$. We will cover $(M, g)$ by areal coordinates $(t, \theta, x, y)$, where the range of the coordinates is $\left(t_{f}, t_{i}\right] \times[0,1]^{3}$ with $0<t_{f}<t_{i}$. The metric is then given by (2) where all functions depend on $t$ and $\theta$ and are periodic in $\theta$ with period 1. The Einstein-Vlasov system implies that the system (23)-(32) completed by (22) holds for all $(t, \theta) \in\left(t_{f}, t_{i}\right] \times[0,1]$. Moreover, we will assume that $f$ does not vanish identically. From what has been said in Section 6, we will prove that for all such $(\mathcal{M}, g, f)$, the hypotheses of Proposition 2 are satisfied, from which Theorem 1 follows immediately.

First we recall some standard facts about the Vlasov field in such spacetimes.

7C. Conservation laws. From the conservation of the Vlasov field $f$ along geodesics, if follows immediately that $f$ is bounded above by some constant $F>0$ :

$$
f \leq F .
$$

Since $v_{2}$ and $v_{3}$ are constant along geodesics, it follows that the support of $f$ in $v_{2}$ and $v_{3}$ is conserved. By compactness of the initial Cauchy surface, we therefore have an upper bound on the support of $f$ in $v_{2}$ and $v_{3}$ in $(\mathcal{M}, g)$. Let $X$ be such an upper bound:

$$
X=\sup \left\{\max \left(\left|v_{2}\right|,\left|v_{3}\right|\right): \exists\left(t, \theta, v_{1}\right) \text { such that } f\left(t, \theta, v_{1}, v_{2}, v_{3}\right)>0\right\}<\infty .
$$

The particle current is given by

$$
N^{\mu}=\frac{\sqrt{\alpha}}{t} \int_{\mathbb{R}^{3}} \frac{f}{\left|v_{0}\right|} v^{\mu} d v_{1} d v_{2} d v_{3} .
$$


From the Vlasov equation it follows that $N^{\mu}$ is divergence free: $\nabla_{\mu} N^{\mu}=0$. We therefore have the conservation law, for all $t$,

$$
\int_{[0,1]} N^{0} t \sqrt{\alpha} e^{2(\nu-U)} d \theta=\int_{[0,1]}\left(\int_{\mathbb{R}^{3}} f d v_{1} d v_{2} d v_{3}\right) d \theta=Q,
$$

for some constant $Q$. And since, by assumption, the Vlasov field does not vanish identically, we have

$$
Q>0 \text {. }
$$

7D. Lower bound on the mean value of $\left|v_{1}\right|$. We now prove a lower bound on the mean value of $\left|v_{1}\right|$ for the measure $f d v d \theta$. This lower bound is the important estimate that takes advantage of the assumption that $f \neq 0$. Coupled to the energy estimates derived in the next section, this estimate will give us uniform control of $\min _{[0,1]} \alpha(t, \cdot)$; see Section $7 \mathrm{H}$.

Lemma 7.1. There exists $\delta>0$ such that

$$
\int_{[0,1]} \int_{\mathbb{R}^{3}} f\left|v_{1}\right| d v_{1} d v_{2} d v_{3} d \theta>\delta
$$

for all $t \in\left(t_{f}, t_{i}\right]$.

Proof. Let

$$
\epsilon=\frac{Q}{16 X^{2} F}
$$

so that $Q-\epsilon 8 X^{2} F=Q / 2>0$. We have

$$
\begin{aligned}
\int_{[0,1]} \int_{\mathbb{R}^{3}} f\left|v_{1}\right| d v_{1} d v_{2} d v_{3} & =\int_{[0,1]} \int_{\mathbb{R}^{2}}\left(\int_{-\epsilon}^{\epsilon} f\left|v_{1}\right| d v_{1}\right) d v_{2} d v_{3}+\int_{[0,1]} \int_{\mathbb{R}^{2}}\left(\int_{\left|v_{1}\right|>\epsilon} f\left|v_{1}\right| d v_{1}\right) d v_{2} d v_{3} \\
& \geq \epsilon \int_{[0,1]} \int_{\mathbb{R}^{2}}\left(\int_{\left|v_{1}\right|>\epsilon} f d v_{1}\right) d v_{2} d v_{3} \geq \epsilon Q / 2
\end{aligned}
$$

7E. Energy estimates. The following energy estimates take their origins in the underlying wave map structure of the equations, visible in the vacuum case [Berger et al. 1997] and easily modifiable to suit the Vlasov case.

Define the energy integral $E_{g, K, \Lambda, f}(t)$ by ${ }^{15}$

$$
E_{g, K, \Lambda, f}=\int_{[0,1]} \frac{v_{t}}{\sqrt{\alpha} t} d \theta
$$

From the constraint equation (23), it follows that

$$
\begin{aligned}
E_{g, K, \Lambda, f}=\int_{[0,1]} \frac{1}{\sqrt{\alpha}}\left(U_{t}^{2}+\right. & \alpha U_{\theta}^{2}+\frac{e^{4 U}}{4 t^{2}}\left(A_{t}^{2}+\alpha A_{\theta}^{2}\right)+\frac{\alpha e^{2 \nu-4 U} J^{2}}{4 t^{2}} \\
& \left.+\frac{\alpha e^{2 v}(K-A J)^{2}}{4 t^{4}}+\alpha e^{2(\nu-U)} \Lambda+8 \pi \frac{\sqrt{\alpha}}{t} \int_{\mathbb{R}^{3}} f\left|v_{0}\right| d v_{1} d v_{2} d v_{3}\right) d \theta
\end{aligned}
$$

\footnotetext{
${ }^{15}$ The motivation for the notation $E_{g, K, \Lambda, f}$ is that this energy may be decomposed into four terms, containing respectively $g, K, \Lambda$ and $f$. Later, we will introduce several other energy integrals and the notation will follow the same pattern.
} 
Using the Einstein equations, we may compute the time derivative of $E_{g, K, \Lambda, f}$ :

$$
\begin{aligned}
\frac{d E_{g, K, \Lambda, f}}{d t}=-\int_{[0,1]}\left[\frac{2}{t}\left(\frac{U_{t}^{2}}{\sqrt{\alpha}}+\frac{e^{4 U}}{4 t^{2}} \sqrt{\alpha} A_{\theta}^{2}\right)+\frac{\sqrt{\alpha} e^{2 v-4 U} J^{2}}{2 t^{3}}+\frac{\sqrt{\alpha} e^{2 v}(K-A J)^{2}}{t^{5}}\right. & \\
& \left.+8 \pi \int_{\mathbb{R}^{3}}\left(\frac{f\left|v_{0}\right|}{t^{2}}+\frac{\alpha e^{2 v} f\left(v_{3}-A v_{2}\right)^{2}}{t^{4}\left|v_{0}\right|}\right) d v_{1} d v_{2} d v_{3}\right] d \theta .
\end{aligned}
$$

Since the right-hand side is nonpositive, $E_{g, K, \Lambda, f}$ is nondecreasing when $t$ is decreasing. ${ }^{16}$

Lemma 7.2. $E_{g, K, \Lambda, f}$ is bounded on $\left(t_{f}, t_{i}\right]$ and admits a continuous extension at $t_{f}$.

Proof. From (39), (41) and the mass shell relation (21), we obtain

$$
\frac{d E_{g, K, \Lambda, f}}{d t} \geq-\frac{4}{t} E_{g, K, \Lambda, f},
$$

where the factor of 4 arises because of the terms containing $(K-A J)^{2}$. Applying Gronwall's lemma and using the lower bound $t \geq t_{f}>0$, we then obtain a uniform bound on $E_{g, K, f}$.

7F. Estimate for $\sqrt{\alpha} e^{2 v+b U}$. Here we exploit the monotonicity properties of the constraint equations.

Lemma 7.3. For any real number $b$,

$$
\sqrt{\alpha} e^{2 v+b U}
$$

is uniformly bounded on $\left(t_{f}, t_{i}\right] \times[0,1]$.

Proof. Using equations (23) and (24), we see that $t^{b^{2} / 8} \sqrt{\alpha} e^{2 v+b U}$ is decreasing with decreasing $t$ :

$$
\begin{aligned}
\partial_{t}\left(t^{b^{2} / 8} \sqrt{\alpha} e^{2 v+b U}\right)= & b^{2} / 8 t^{b^{2} / 8-1} \sqrt{\alpha} e^{2 v+b U}+t^{b^{2} / 8} \frac{\alpha_{t}}{2 \sqrt{\alpha}} e^{2 v+b U}+t^{b^{2} / 8} \sqrt{\alpha} e^{2 v+b U}\left(2 v_{t}+b U_{t}\right) \\
= & t^{b^{2} / 8} \sqrt{\alpha} e^{2 v+b U}\left(2 t\left[\left(U_{t}+\frac{b}{4 t}\right)^{2}+\alpha U_{\theta}^{2}+\frac{e^{4 U}}{4 t^{2}}\left(A_{t}^{2}+\alpha A_{\theta}^{2}\right)\right]\right. \\
& \left.+8 \pi \sqrt{\alpha} \int_{\mathbb{R}^{3}} f\left(\left|v_{0}\right|+\frac{\alpha v_{1}^{2}}{\left|v_{0}\right|}\right) d v_{1} d v_{2} d v_{3}\right) \geq 0 .
\end{aligned}
$$

Thanks to the freedom in the choice of the Killing fields, we also have:

Lemma 7.4. For any positive real number $r$ and any real number $\lambda$,

$$
\alpha^{r / 2} e^{2 r v+\lambda U} A^{2}
$$

is bounded on $\left(t_{f}, t_{i}\right] \times[0,1]$.

Proof. Consider inverting the role of $X$ and $Y$ in the metric:

$$
\begin{aligned}
\tilde{X} & =Y, \\
\tilde{Y} & =-X .
\end{aligned}
$$

\footnotetext{
${ }^{16}$ In contrast, $E_{g}=\int_{[0,1]} \frac{1}{\sqrt{\alpha}}\left(U_{t}^{2}+\alpha U_{\theta}^{2}+\frac{e^{4 U}}{4 t^{2}}\left(A_{t}^{2}+\alpha A_{\theta}^{2}\right)\right) d \theta$ is not necessarily monotonic.
} 
This is an $\operatorname{SL}(2, \mathbb{R})$ transformation and therefore (see Section $2 \mathrm{~A}$ ), the form of the metric is unchanged if we relabel the metric functions as follows, using tilde notations for the new metric functions:

$$
\begin{aligned}
e^{2 \tilde{U}} & =e^{2 U} A^{2}+t^{2} e^{-2 U}, \\
e^{2 \tilde{U}} \tilde{A} & =-A e^{2 U}, \\
\tilde{\alpha} & =\alpha \\
\tilde{\alpha} e^{2(\tilde{v}-\tilde{U})} & =\alpha e^{2(v-U)} .
\end{aligned}
$$

Let $q<r$, using the previous equations, it follows that

$$
\tilde{\alpha}^{q / 2} e^{2 q \tilde{v}+2(1-q) \tilde{U}}=\alpha^{q / 2} e^{2 q v+2(1-q) U} A^{2}+\alpha^{q / 2} t^{2} e^{2 q \nu-2(1+q) U}
$$

Since the tilde metric functions satisfy the same equations with respect to the same $t$, the left-hand side of $(51)$ is bounded on $\left(t_{f}, t_{i}\right] \times[0,1]$ from Lemma 7.3 . Since the second term on the right hand side is positive, the first term is bounded. By Lemma 7.3,

$$
\alpha^{(r-q) / 2} e^{2(r-q) \nu+(\lambda-2(1-q)) U}
$$

is bounded, and multiplying this by the first term on the right in (51), we obtain the desired estimate.

The quantity $\sqrt{\alpha} e^{v}$ will play an important role in the analysis. To simplify some of the computations, let us define $\beta$ by

$$
e^{\beta}=\sqrt{\alpha} e^{v}
$$

7G. Estimates for spatial derivatives integrals. From (25), we derive

$$
\beta_{\theta}=2 t\left(U_{t} U_{\theta}+\frac{e^{4 U}}{4 t^{2}} A_{t} A_{\theta}\right)-8 \pi \sqrt{\alpha} \int_{\mathbb{R}^{3}} f v_{1} d v_{1} d v_{2} d v_{3}
$$

It follows from this equation and the energy estimates obtained in Lemma 7.2 that we can uniformly control the variation in $\theta$ of the metric functions. In other words:

Lemma 7.5. The integrals

$$
\int_{[0,1]}\left|\beta_{\theta}\right| d \theta, \quad \int_{[0,1]}\left|U_{\theta}\right| d \theta, \quad \int_{[0,1]} e^{2 U}\left|A_{\theta}\right| d \theta, \quad \int_{[0,1]}\left|J_{\theta}\right| d \theta, \quad \int_{[0,1]}\left|K_{\theta}\right| d \theta
$$

are uniformly bounded on $\left(t_{f}, t_{i}\right]$.

Proof. From (54), we obtain

$$
\frac{\left|\beta_{\theta}\right|}{t} \leq \frac{v_{t}}{\sqrt{\alpha} t}
$$

and by integration we obtain a bound on $\int_{[0,1]}\left|\beta_{\theta}\right| d \theta$ in view of (39) and the bound on $E_{g, K, \Lambda, f}$. The bounds on $\int_{[0,1]}\left|J_{\theta}\right| d \theta$ and $\int_{[0,1]}\left|K_{\theta}\right| d \theta$ follow from the auxiliary equations (30), (32), and the conservation of the flux (36) together with (34). The bounds on the remaining quantities follow from the definition of $E_{g, K, f}$ and the monotonicity in $t$ of $\alpha$. 
7H. Control of $\alpha$ along special curves. In this section, we obtain a bound on $\min _{\theta \in[0,1]} \alpha(t, \cdot)$, using the lower bound on the mean value of $\left|v_{1}\right|$.

Lemma 7.6. $\min _{\theta \in[0,1]} \alpha(t, \cdot)$ is uniformly bounded on $\left(t_{f}, t_{i}\right]$.

Proof. From the definition of $E_{g, K, \Lambda, f}$, we have

$$
8 \pi \int_{[0,1]} \int_{\mathbb{R}^{3}} f\left|v_{0}\right| d v_{1} d v_{2} d v_{3} d \theta \leq t E_{g, K, \Lambda, f},
$$

and from the mass shell relation (21), we obtain

$$
\begin{aligned}
\int_{[0,1]} \int_{\mathbb{R}^{3}} f \sqrt{\alpha}\left|v_{1}\right| d v_{1} d v_{2} d v_{3} d \theta & \leq \frac{t E_{g, K, \Lambda, f}}{8 \pi}, \\
\sqrt{\min _{\theta \in[0,1]} \alpha(t, \cdot)} \int_{[0,1]} \int_{\mathbb{R}^{3}} f\left|v_{1}\right| d v_{1} d v_{2} d v_{3} d \theta & \leq \frac{t E_{g, K, \Lambda, f}}{8 \pi}, \\
\sqrt{\min _{\theta \in[0,1]} \alpha(t, \cdot)} & \leq \frac{t E_{g, K, \Lambda, f}}{8 \pi \delta},
\end{aligned}
$$

where we have used the lower bound of Lemma 7.1 to obtain the last inequality.

Remark 7H.1. This is the only step in the proof of Theorem 1 where we need the assumption that $f$ does not vanish. In particular, in the proof by contradiction of Theorem 2 given in Section 8, we will be able to assume that the above lemma does not hold (see Section 8D).

Corollary 1. There exists $\bar{\theta} \in[0,1]$ such that $\alpha(t, \bar{\theta})$ is bounded on $\left(t_{f}, t_{i}\right]$.

Proof. Let $M$ be a bound for $\min _{[0,1]} \alpha$. Suppose that for every $\theta \in[0,1], \alpha(t, \theta)$ is unbounded. By assumption, for every $\theta$, there exists a $t^{*}(\theta)$, for which $\alpha\left(t^{*}(\theta), \theta\right)>2 M$ and by continuity, there exists an open interval $I_{\theta}=\left(\theta-\delta_{\theta}, \theta+\delta_{\theta}\right)$ such that

$$
\alpha\left(t^{*}(\theta), \theta^{\prime}\right)>M \text { for all } \theta^{\prime} \in I_{\theta} .
$$

Consider $\bigcup_{\theta \in[0,1]} I_{\theta}$. This is an open cover of $[0,1]$; by compactness, it has a finite subcover. Let $\theta_{0}, \theta_{1}, \ldots, \theta_{n}$ be such that $[0,1]=\bigcup_{0 \leq k \leq n} I_{\theta_{k}}$ and let $T=\min _{0 \leq k \leq n} t^{*}\left(\theta_{k}\right)$. Since $\alpha$ is increasing with decreasing time, it follows that $\alpha(T, \theta)>M$ for every $\theta \in[0,1]$ which contradicts the definition of $M$.

\section{I. Estimate for $\sqrt{\alpha} e^{v+b U}$.}

Lemma 7.7. For any real number $b, \sqrt{\alpha} e^{\nu+b U}=e^{\beta+b U}$ is uniformly bounded on $\left(t_{f}, t_{i}\right] \times[0,1]$.

Proof. By Lemma 7.3 and Corollary 1, we have

$$
e^{2 \beta(t, \bar{\theta})+b U(t, \bar{\theta})}=\sqrt{\alpha(t, \bar{\theta})} \sqrt{\alpha(t, \bar{\theta})} e^{2 v(t, \bar{\theta})+b U(t, \bar{\theta})} \leq B,
$$

for some constant $B>0$. The uniform bound on $e^{\beta+b U}$ then follows from Lemma 7.5, since we have

$$
\begin{aligned}
|\beta(t, \bar{\theta})-\beta(t, \theta)| & \leq B^{\prime}, \\
|U(t, \bar{\theta})-U(t, \theta)| & \leq B^{\prime},
\end{aligned}
$$

for all $(t, \theta) \in\left(t_{f}, t_{i}\right] \times[0,1]$ and a fixed constant $B^{\prime}>0$, which implies

$$
e^{\beta(t, \theta)+b U(t, \theta)} \leq B e^{B^{\prime}+|b| B^{\prime}} .
$$


7J. Control of the polarization. Corollary 1 also implies a sharper estimate on the inner product of the Killing fields:

Lemma 7.8. For any real numbers $r$ and $b, e^{r \beta+b U} A$ is uniformly bounded on $\left(t_{f}, t_{i}\right] \times[0,1]$.

Proof. It follows from Corollary 1 and Lemma 7.4 that $e^{r \beta+b U} A$ is bounded on $\left(t_{f}, t_{I}\right] \times\{\bar{\theta}\}$. Furthermore,

$$
e^{r \beta+b U} A(t, \theta) \leq e^{r \beta+b U} A(t, \bar{\theta})+\int_{\bar{\theta}}^{\theta}\left(e^{r \beta+b U} A\left(r \beta_{\theta}+b U_{\theta}\right)+e^{r \beta+(b-2) U} e^{2 U} A_{\theta}\right) d \theta^{\prime} .
$$

Using the bound on $e^{r \beta+b U} A(t, \bar{\theta})$, we therefore obtain

$$
\left|e^{r \beta+b U} A(t, \theta)\right| \leq B+\left|\int_{\bar{\theta}}^{\theta} e^{r \beta+b U}\right| A\left|\left(\left|r \beta_{\theta}\right|+\left|b U_{\theta}\right|\right) d \theta\right| .
$$

for some constant $B>0$ and we can conclude using Gronwall's inequality and Lemma 7.5.

7K. Estimates for the time integrals of the twist quantities. To estimate the first derivatives of $U$ and $A$ in the next section, we will need the following estimates for the time integrals of the twist quantities:

Lemma 7.9. The quantity

$$
\int_{t}^{t_{i}} \max _{\theta \in[0,1]}\left[e^{2 \beta-4 U} J^{2}\right]\left(t^{\prime}, \theta\right) d t^{\prime}
$$

is uniformly bounded on $\left(t_{f}, t_{i}\right]$.

Proof. From Lemma 7.5, there exists a constant $M$ such that

$$
\left|J\left(t^{\prime}, \theta\right)\right| \leq M+\left|J\left(t^{\prime}, \bar{\theta}\right)\right| \quad \text { and } \quad e^{2 \beta-4 U}\left(t^{\prime}, \theta\right) \leq e^{6 M} e^{2 \beta-4 U}\left(t^{\prime}, \bar{\theta}\right) .
$$

Thus,

$$
\int_{t}^{t_{i}} \max _{\theta \in[0,1]}\left[e^{2 \beta-4 U} J^{2}\right]\left(t^{\prime}, \theta\right) d t^{\prime} \leq \int_{t}^{t_{i}} e^{6 M}\left(e^{2 \beta-4 U}\left(J^{2}+2 M|J|+M^{2}\right)\right)\left(t^{\prime}, \bar{\theta}\right) d t^{\prime}
$$

and using $2|J| \leq J^{2}+1$ as well as Lemma 7.7, we obtain

$$
\int_{t}^{t_{i}} \max _{\theta \in[0,1]}\left[e^{2 \beta-4 U} J^{2}\right]\left(t^{\prime}, \theta\right) d t^{\prime} \leq B+B^{\prime} \int_{t}^{t_{i}}\left[e^{2 \beta-4 U} J^{2}\right]\left(t^{\prime}, \bar{\theta}\right) d t^{\prime},
$$

for some constants $B$ and $B^{\prime}$.

Since by integration of (24) we have

$$
\int_{t}^{t_{i}}\left[e^{2 \beta-4 U} J^{2}\right]\left(t^{\prime}, \bar{\theta}\right) d t^{\prime} \leq t_{i} \ln \frac{\alpha(t, \bar{\theta})}{\alpha\left(t_{i}, \bar{\theta}\right)},
$$

which is bounded from Corollary 1, the right-hand side of (68) is uniformly bounded.

Similarly:

Lemma 7.10. The quantity

$$
\int_{t}^{t_{i}} \max _{\theta \in[0,1]}\left[e^{2 \beta}(K-A J)^{2}\right]\left(t^{\prime}, \theta\right) d t^{\prime}
$$

is uniformly bounded on $\left(t_{f}, t_{i}\right]$. 
Proof. We first integrate the $\theta$ derivative of $e^{\beta}(K-A J)$, using the auxiliary equations (32) and (30) to replace the derivatives of $K_{\theta}$ and $J_{\theta}$ by matter terms:

$$
\begin{aligned}
& e^{\beta}|K-A J|(t, \theta) \\
& \leq e^{\beta}|K-A J|(t, \bar{\theta})+\mid \int_{\bar{\theta}}^{\theta}\left(e^{\beta}|K-A J|\left|\beta_{\theta}\right|+e^{\beta-2 U}|J| e^{2 U}\left|A_{\theta}\right|\right. \\
& \left.+16 \pi e^{\beta} \int_{\mathbb{R}^{3}} f\left|v_{3}\right| d v_{1} d v_{2} d v_{3}+16 \pi e^{\beta} A \int_{\mathbb{R}^{3}} f\left|v_{2}\right| d v_{1} d v_{2} d v_{3}\right) d \theta^{\prime} \mid \text {. }
\end{aligned}
$$

Using Lemmas 7.5, 7.7 and 7.8, as well as the conservation law (36) and the uniform boundedness (34) of the support of $f$ in $v_{3}$ and $v_{2}$, we obtain

$$
e^{\beta}|K-A J|(t, \theta) \leq e^{\beta}|K-A J|(t, \bar{\theta})+B\left|\int_{\bar{\theta}}^{\theta} e^{\beta}\right| K-A J|| \beta_{\theta}\left|d \theta^{\prime}\right|+C \max _{\theta \in[0,1]}\left[e^{\beta-2 U}|J|(t, \cdot)\right]+D,
$$

for some constants $B, C$ and $D$. Applying Gronwall's lemma, we obtain

$$
e^{\beta}|K-A J|(t, \theta) \leq\left(e^{\beta}|K-A J|(t, \bar{\theta})+B \max _{\theta \in[0,1]}\left[e^{\beta-2 U}|J|(t, \cdot)\right]+C\right)\left(1+e^{\left|\int_{\bar{\theta}}^{\theta}\right| \beta_{\theta}\left|d \theta^{\prime}\right|}\right)
$$

and therefore, using Lemma 7.5 again, we have

$$
\max _{\theta \in[0,1]} e^{\beta}|K-A J|(t, \cdot) \leq D\left(e^{\beta}|K-A J|(t, \bar{\theta})+B \max _{\theta \in[0,1]}\left[e^{\beta-2 U}|J|(t, \cdot)\right]+C\right) .
$$

We now conclude by integrating (24) and applying Lemma 7.9 to bound the term containing $J$.

7L. Null cone estimates for the first derivatives of $U$ and $A$ coupled to an estimate for the support of $\boldsymbol{f}$. We will perform null cone energy estimates to bound the first derivatives of $U$ and $A$. However, to close the estimates we will also need to estimate the support of $f$.

Recall the definition of the energy density:

$$
g=U_{t}^{2}+\alpha U_{\theta}^{2}+\frac{e^{4 U}}{4 t^{2}}\left(A_{t}^{2}+\alpha A_{\theta}^{2}\right)
$$

Lemma 7.11. The function $g$ is uniformly bounded on $\left(t_{f}, t_{i}\right] \times[0,1]$ and the support of $f$ is uniformly bounded on $\left(t_{f}, t_{i}\right] \times[0,1] \times \mathbb{R}^{3}$.

Proof. We define $g^{\times}$by

$$
g^{\times}=2 \sqrt{\alpha}\left(U_{t} U_{\theta}+\frac{e^{4 U}}{4 t^{2}} A_{t} A_{\theta}\right) .
$$

We have $g \pm g^{\times} \geq 0$. Let $\partial_{u}=\partial_{t}-\sqrt{\alpha} \partial_{\theta}$ and $\partial_{v}=\partial_{t}+\sqrt{\alpha} \partial_{\theta}$.

Using the Einstein equations, we can compute the null derivatives of $g+g^{\times}$and $g-g^{\times}$:

$$
\begin{aligned}
\partial_{u}\left(g+g^{\times}\right)= & -\frac{2}{t}\left(U_{t}^{2}+\frac{e^{4 U}}{4 t^{2}} \alpha A_{\theta}^{2}\right)+\frac{\alpha_{t}}{\alpha}\left(g+g^{\times}\right)-\frac{g^{\times}}{t} \\
& +2\left(U_{t}+\sqrt{\alpha} U_{\theta}\right)\left(\frac{e^{2 \beta-4 U}}{2 t^{2}} J^{2}+8 \pi \frac{\sqrt{\alpha} e^{2 \beta-2 U}}{2 t} \int_{\mathbb{R}^{3}} \frac{f\left(1+2 e^{-2 U} v_{2}^{2}\right)}{\left|v_{0}\right|} d v_{1} d v_{2} d v_{3}+e^{2 \beta-2 U} \Lambda\right) \\
& +\frac{e^{4 U}}{2 t^{2}}\left(A_{t}+\sqrt{\alpha} A_{\theta}\right)\left(\frac{e^{2 \beta-4 U}}{t^{2}} J(K-A J)+16 \pi \sqrt{\alpha} \frac{e^{2 \beta-4 U}}{t} \int_{\mathbb{R}^{3}} \frac{f v_{2}\left(v_{3}-A v_{2}\right)}{\left|v_{0}\right|} d v_{1} d v_{2} d v_{3}\right)
\end{aligned}
$$




$$
\begin{aligned}
\partial_{u}\left(g-g^{\times}\right)= & -\frac{2}{t}\left(U_{t}^{2}+\frac{e^{4 U}}{4 t^{2}} \alpha A_{\theta}^{2}\right)+\frac{\alpha_{t}}{\alpha}\left(g-g^{\times}\right)+\frac{g^{\times}}{t} \\
& +2\left(U_{t}-\sqrt{\alpha} U_{\theta}\right)\left(\frac{e^{2 \beta-4 U}}{2 t^{2}} J^{2}+8 \pi \frac{\sqrt{\alpha} e^{2 \beta-2 U}}{2 t} \int_{\mathbb{R}^{3}} \frac{f\left(1+2 e^{-2 U} v_{2}^{2}\right)}{\left|v_{0}\right|} d v_{1} d v_{2} d v_{3}+e^{2 \beta-2 U} \Lambda\right) \\
& +\frac{e^{4 U}}{2 t^{2}}\left(A_{t}-\sqrt{\alpha} A_{\theta}\right)\left(\frac{e^{2 \beta-4 U}}{t^{2}} J(K-A J)+16 \pi \sqrt{\alpha} \frac{e^{2 \beta-4 U}}{t} \int_{\mathbb{R}^{3}} \frac{f v_{2}\left(v_{3}-A v_{2}\right)}{\left|v_{0}\right|} d v_{1} d v_{2} d v_{3}\right) .
\end{aligned}
$$

Define $T_{1}$ and $T_{2}$ by

$$
\begin{aligned}
& T_{1}=\frac{e^{2 \beta-4 U}}{2 t^{2}} J^{2}+8 \pi \frac{\sqrt{\alpha} e^{2 \beta-2 U}}{2 t} \int_{\mathbb{R}^{3}} \frac{f\left(1+2 e^{-2 U} v_{2}^{2}\right)}{\left|v_{0}\right|} d v_{1} d v_{2} d v_{3}+e^{2 \beta-2 U} \Lambda, \\
& T_{2}=\frac{e^{2 \beta-2 U}}{t^{3}} J(K-A J)+16 \pi \sqrt{\alpha} \frac{e^{2 \beta-2 U}}{t^{2}} \int_{\mathbb{R}^{3}} \frac{f v_{2}\left(v_{3}-A v_{2}\right)}{\left|v_{0}\right|} d v_{1} d v_{2} d v_{3} .
\end{aligned}
$$

$T_{1}$ and $T_{2}$ can be estimated using (24):

$$
\begin{aligned}
& \left|T_{1}\right| \leq\left|\frac{\alpha_{t}}{2 t \alpha}\right|, \\
& \left|T_{2}\right| \leq\left|\frac{\alpha_{t}}{2 \alpha}\right|
\end{aligned}
$$

We therefore obtain

$$
\begin{aligned}
& \left|\partial_{u}\left(g+g^{\times}\right)\right| \leq\left|\frac{\alpha_{t}}{\alpha}\right|\left(\frac{g}{t}+\frac{1}{2 t}+2 g\right)+\frac{3 g}{t}, \\
& \left|\partial_{v}\left(g-g^{\times}\right)\right| \leq\left|\frac{\alpha_{t}}{\alpha}\right|\left(\frac{g}{t}+\frac{1}{2 t}+2 g\right)+\frac{3 g}{t} .
\end{aligned}
$$

To perform null cone estimates, in view of the last two inequalities, we need to control the time integral of $\alpha_{t} / \alpha$, that is to say, we need to control $\ln \alpha$. Consider the right-hand side of (24). The time integral of the two terms containing the twist quantities are bounded from Lemmas 7.9, 7.10 and the term containing the cosmological constant is bounded from Lemma 7.7. Therefore to control $\left|\alpha_{t} / \alpha\right|$, we only need to control the last term, which is the term containing the Vlasov field. While we already have a bound on the support of the Vlasov field in $v_{2}$ and $v_{3}$, we still cannot estimate the support of $f$ in $v_{1}$. Therefore, the best we can obtain from (24) is an estimate for $\left|\alpha_{t} / \alpha\right|$ which depends on the support of $f$ in $v_{1}$ and quantities which have been shown to be bounded. On the other hand, using the characteristic equations associated with the Vlasov equation, i.e., using the geodesic equations, we can obtain a bound on the support of $v_{1}$ in terms of $g$ and quantities which have been shown to be bounded. The strategy, which was originally developed by Andréasson [1999], is therefore to combine the two. For this, we define the functions

$$
\begin{aligned}
u_{1} & =\sqrt{\alpha} v_{1}, \\
\bar{u}_{1}(t) & =\sup \left\{\sqrt{\alpha}\left|v_{1}\right|: \exists\left(t^{\prime}, \theta, v_{2}, v_{3}\right) \in\left[t, t_{i}\right] \times[0,1] \times \mathbb{R}^{2} \text { such that } f\left(t^{\prime}, \theta, v_{1}, v_{2}, v_{3}\right) \neq 0\right\}, \\
\psi(t) & =\max \left(\sup _{\theta \in[0,1]} g(t, \cdot)+\bar{u}_{1}^{2}(t), 2\right) .
\end{aligned}
$$


We start by estimating $\left|\frac{\alpha_{t}}{\alpha}\right|=-\frac{\alpha_{t}}{\alpha}$ in terms of $\bar{u}_{1}$ :

$$
-\frac{\alpha_{t}}{\alpha}(t, \theta) \leq C(t)+B(t, \theta)
$$

for some nonnegative function $C(t)$ whose integral in time is bounded and where $B(t, \theta)$ is given by

$$
B(t, \theta)=16 \pi e^{2 \beta-2 U} \int_{\mathbb{R}^{3}} \frac{f\left(1+e^{-2 U} v_{2}^{2}+t^{-2} e^{2 U}\left(v_{3}-A v_{2}\right)^{2}\right)}{\left|v_{0}\right|} d u_{1} d v_{2} d v_{3} .
$$

We have

$$
\begin{aligned}
& B(t, \theta) \\
& \leq 16 \pi e^{2 \beta-2 U} \int_{\mathbb{R}^{3}} \frac{f\left(1+e^{-2 U} v_{2}^{2}+t^{-2} e^{2 U}\left(v_{3}-A v_{2}\right)^{2}\right)}{e^{\beta-U} \sqrt{1+e^{-2 \beta+2 U} u_{1}^{2}}} d u_{1} d v_{2} d v_{3} \\
& \leq 16 \pi e^{\beta-U} F\left(1+e^{-2 U} X^{2}+\frac{e^{2 U}}{t^{2}}(X+|A| X)^{2}\right) 4 X^{2} \int_{-\bar{u}_{1}}^{\bar{u}_{1}} \frac{d u_{1}}{\sqrt{1+e^{-2 \beta+2 U} u_{1}^{2}}} \\
& \leq 16 \pi e^{\beta-U} F\left(1+e^{-2 U} X^{2}+\frac{e^{2 U}}{t^{2}}(X+|A| X)^{2}\right) 4 X^{2} \cdot 2\left(e^{\beta-U} \ln \left(\bar{u}_{1}+\sqrt{e^{2 \beta-2 U}+{\overline{u_{1}}}^{2}}\right)+e^{-1}\right) .
\end{aligned}
$$

Therefore, using Lemmas 7.7 and 7.8, it follows from (86) that there exist a nonnegative function $C(t)$ whose integral in time is bounded and a constant $D>0$ such that we have the estimate

$$
-\frac{\alpha_{t}}{\alpha}(t, \theta) \leq C(t)+D \ln \left(1+\bar{u}_{1}^{2}\right)
$$

On the other hand, from the characteristic equation of the Vlasov equation (22), it follows that

$$
\begin{aligned}
\frac{d u_{1}^{2}}{d s}=\frac{\alpha_{t}}{\alpha} & u_{1}^{2} \\
& +\frac{2 \sqrt{\alpha} u_{1}}{v_{0}}\left(e^{2 \beta-2 U}\left(\beta_{\theta}-U_{\theta}\right)+e^{2 \beta-4 U}\left(\beta_{\theta}-2 U_{\theta}\right) v_{2}^{2}+\frac{e^{2 \beta}}{t^{2}}\left(v_{3}-A v_{2}\right)\left(\left(v_{3}-A v_{2}\right) \beta_{\theta}-A_{\theta} v_{2}\right)\right) \\
& +\frac{2 e^{2 \beta} u_{1}}{t}\left(\frac{(K-A J)\left(v_{3}-A v_{2}\right)}{t^{2}}+e^{-4 U} J v_{2}\right)
\end{aligned}
$$

and therefore we have, by integration,

$$
\begin{array}{r}
\left|u_{1}^{2}(s)-u_{1}^{2}\left(t_{i}\right)\right|=\mid \int_{t_{i}}^{s}\left(\frac{\alpha_{t}}{\alpha} u_{1}^{2}+\frac{2 \sqrt{\alpha} u_{1}}{v_{0}}\left(e^{2 \beta-2 U}\left(\beta_{\theta}-U_{\theta}\right)+e^{2 \beta-4 U}\left(\beta_{\theta}-2 U_{\theta}\right) v_{2}^{2}\right.\right. \\
\left.+\frac{e^{2 \beta}}{t^{2}}\left(v_{3}-A v_{2}\right)\left(\left(v_{3}-A v_{2}\right) \beta_{\theta}-A_{\theta} v_{2}\right)\right) \\
\left.+\frac{2 e^{2 \beta} u_{1}}{t}\left(\frac{(K-A J)\left(v_{3}-A v_{2}\right)}{t^{2}}+e^{-4 U} J v_{2}\right)\right) d s^{\prime} \mid
\end{array}
$$

Let us estimate one by one the terms on the right-hand side. 
The first term can be estimated using (88) as follows. ${ }^{17}$ For $s<t_{i}$, we have

$$
\left|\int_{t_{i}}^{s} \frac{\alpha_{t}}{\alpha} u_{1}^{2} d s^{\prime}\right| \leq\left|\int_{t_{i}}^{s}\right| \frac{\alpha_{t}}{\alpha} \bar{u}_{1}^{2}\left(s^{\prime}\right)\left|d s^{\prime}\right| \leq \mid \int_{t_{i}}^{s}\left(C(s)+D \ln \left(1+\bar{u}_{1}^{2}\left(s^{\prime}\right)\right) \bar{u}_{1}^{2}\left(s^{\prime}\right) d s^{\prime} \mid,\right.
$$

where $C(s)$ is a nonnegative function whose integral is uniformly bounded and $D$ is a nonnegative constant.

To estimate the second term on the right-hand side of (90), we use (54) to obtain

$$
\sqrt{\alpha}\left|\beta_{\theta}\right| \leq B g+D \bar{u}_{1}^{2},
$$

for some constants $B$ and $D$ which depend on the bounds on $t, f$ and the support of $f$ in $v_{2}$ and $v_{3}$. Moreover, from the definition of $g$, we have

$$
\begin{aligned}
\sqrt{\alpha}\left|U_{\theta}\right| & \leq \frac{g}{2}+\frac{1}{2}, \\
\sqrt{\alpha} \frac{e^{2 U}\left|A_{\theta}\right|}{t} & \leq 2 g+\frac{1}{2} .
\end{aligned}
$$

From the uniform bounds on $e^{2 \beta-2 U}, e^{2 \beta} A$, and the support of $f$ in $v_{2}$ and $v_{3}$, and from the estimate for $\beta_{\theta}$, we have, using $\left|u_{1}\right| \leq\left|v_{0}\right|$, that along a characteristic for which $f$ does not uniformly vanish:

$$
\begin{array}{r}
\left|\int_{t_{i}}^{s} \frac{2 \sqrt{\alpha} u_{1}}{v_{0}}\left(e^{2 \beta-2 U}\left(\beta_{\theta}-U_{\theta}\right)+e^{2 \beta-4 U}\left(\beta_{\theta}-2 U_{\theta}\right) v_{2}^{2}+\frac{e^{2 \beta}}{t^{2}}\left(v_{3}-A v_{2}\right)\left(\left(v_{3}-A v_{2}\right) \beta_{\theta}-A_{\theta} v_{2}\right)\right) d s^{\prime}\right| \\
\leq B+\left|\int_{t_{i}}^{s}\left(D g+E \bar{u}_{1}^{2}\right) d s^{\prime}\right|,
\end{array}
$$

for some constants $B, D$ and $E$.

Consider the last term on the right-hand side of (90). We have

$$
\begin{aligned}
& \left|\int_{t_{i}}^{s} \frac{2 e^{2 \beta} u_{1}}{t^{3}}\left((K-A J)\left(v_{3}-A v_{2}\right)+e^{-4 U} J v_{2}\right) d s^{\prime}\right| \\
& \quad \leq\left|\int_{t_{i}}^{s} \frac{2 \bar{u}_{1}}{t_{f}^{3}}\left(e^{\beta} \max _{\theta \in[0,1]}\left(e^{\beta}|K-A J|\right)(t, \cdot)|X+| A|X|+e^{\beta-2 U} X \max _{\theta \in[0,1]}\left(e^{\beta-2 U}|J|\right)(t, \cdot)\right) d s^{\prime}\right| .
\end{aligned}
$$

Using Lemmas 7.7, 7.8, 7.9, 7.10 and the inequality $2 a \leq a^{2}+1$ to replace $\bar{u}_{1}, \max _{\theta \in[0,1]}\left(e^{\beta}|K-A J|\right)(t, \cdot)$ and $\max _{\theta \in[0,1]}\left(e^{\beta-2 U}|J|\right)(t, \cdot)$ by their respective squares, we obtain

$$
\left|\int_{t_{i}}^{s} \frac{2 e^{2 \beta} u_{1}}{t^{3}}\left((K-A J)\left(v_{3}-A v_{2}\right)+e^{-4 U} J v_{2}\right) d s^{\prime}\right| \leq B+\left|\int_{t_{i}}^{s} \bar{u}_{1}^{2} F(s) d s^{\prime}\right|,
$$

where $B$ is a constant and $F(s)$ is a nonnegative function whose integral is uniformly bounded. Using (91), (95) and (97), we therefore obtain for $\bar{u}_{1}$ the estimate

$$
\bar{u}_{1}^{2}(t) \leq B+\int_{t_{i}}^{s}\left(C(s)+B \ln \left(1+\bar{u}_{1}^{2}\left(s^{\prime}\right)\right)\right) \bar{u}_{1}^{2} d s^{\prime}+\int_{t_{i}}^{s}\left(B g+B \bar{u}_{1}^{2}\right) d s^{\prime}+\int_{t_{i}}^{s} \bar{u}_{1}^{2} F(s) d s^{\prime} .
$$

${ }^{17}$ Note the importance of the independence in $\theta$ of the right-hand side of (88) to perform the estimate along the characteristics. 
where $B$ is a nonnegative constant and $C(s), F(s)$ are nonnegative function whose integrals are uniformly bounded.

These estimates are sufficient to obtain an upper bound on $\psi$. We first use equations (81) and (82) to do a null cone estimate for $g(t, \theta)$. For this let $(t, \theta)$ be in $\left(t_{f}, t_{i}\right] \times[0,1]$ and integrate (81) and (82) along the integral curves of $\partial_{u}, \partial_{v}$ ending at $(t, \theta)$. Adding the equations obtained, we have

$$
2 g(t, \theta) \leq B+\int_{u}\left|\frac{\alpha_{t}}{\alpha}\right|\left(\left(\frac{2 g}{t}+1+2 g\right)+\frac{3 g}{t}\right) d u^{\prime}+\int_{v}\left|\frac{\alpha_{t}}{\alpha}\right|\left(\left(\frac{2 g}{t}+1+2 g\right)+\frac{3 g}{t}\right) d v^{\prime} .
$$

where $B$ is a constant which depends on the maximum of $g$ on the initial hypersurface and is finite by compactness. Using the estimate (88) and taking the maximum for $\theta$ in $[0,1]$, we obtain, for $t \in\left(t_{f}, t_{i}\right]$,

$$
\max _{\theta \in[0,1]} g(t, \cdot) \leq B+\int_{t}^{t_{i}}\left(C\left(t^{\prime}\right)+B \ln \left(1+\bar{u}_{1}^{2}\left(t^{\prime}\right)\right) \max _{\theta \in[0,1]} g\left(t^{\prime}, \cdot\right) d t^{\prime},\right.
$$

where $B$ is a nonnegative constant and $C(t)$ is a nonnegative function whose integral is uniformly bounded. Combining this with (98), we derive for $\psi$ the estimate

$$
\psi(t) \leq B+\int_{t}^{t_{i}} F(s) \ln (\psi)(s) \psi(s) d s,
$$

where $B$ is nonnegative constant and $F(s)$ is a nonnegative function whose integral is uniformly bounded. From the last line it follows that

$$
F \psi \ln \psi\left(B+\int_{t}^{t_{i}} F(s) \ln (\psi)(s) \psi(s) d s\right)^{-1}\left(\ln \left(B+\int_{t}^{t_{i}} F(s) \ln (\psi)(s) \psi(s) d s\right)\right)^{-1} \leq F(s),
$$

and by integration we obtain

$$
\psi(t) \leq B^{\exp \int_{t}^{t_{i}} F(s) d s} .
$$

Since the integral is uniformly bounded, it follows that $\psi$ is uniformly bounded.

7M. Continuous extension of the metric functions. Now that $g$ and the support of $f$ have been proven to be uniformly bounded, it follows easily that:

Lemma 7.12. The first derivatives of $U, A, J, K$, together with $v_{t}, \alpha_{t}$ are uniformly bounded on $\left(t_{f}, t_{i}\right] \times$ $[0,1]$ and $U, A, v, \alpha, J, K$ admit continuous extension to $t=t_{f}$.

7N. Estimates for the derivatives of $f, v_{\theta}, \alpha_{\theta}$ and higher order estimates. Such estimates follow by standard methods, which can be found for instance in [Weaver 2004].

70. The conclusion. Since all metric functions, the Vlasov field and all their derivatives have been shown to be uniformly bounded, the assumptions of Proposition 2 have been retrieved. In particular, the maximal Cauchy development cannot have $t_{f}>0$ which concludes the proof of Theorem 1 .

\section{Proof of Theorem 2}

8A. The Einstein equations in areal coordinates for vacuum $T^{2}$-symmetric spacetimes. The Einstein equations (1) for vacuum $T^{2}$-symmetric solutions reduce in areal coordinates to the following system of equations: 
Constraint equations:

$$
\begin{aligned}
\frac{v_{t}}{t} & =U_{t}^{2}+\alpha U_{\theta}^{2}+\frac{e^{4 U}}{4 t^{2}}\left(A_{t}^{2}+\alpha A_{\theta}^{2}\right)+\frac{\alpha e^{2 v} K^{2}}{4 t^{4}}+\alpha e^{2(v-U)} \Lambda, \\
\frac{v_{\theta}}{t} & =2 U_{t} U_{\theta}+\frac{e^{4 U}}{2 t^{2}} A_{t} A_{\theta}-\frac{\alpha_{\theta}}{2 t \alpha}, \\
\frac{\alpha_{t}}{\alpha} & =-4 t \alpha e^{2(v-U)} \Lambda-\frac{\alpha e^{2 v} K^{2}}{t^{3}} .
\end{aligned}
$$

Evolution equations:

$$
\begin{aligned}
v_{t t}-\alpha v_{\theta \theta} & =\frac{\alpha_{\theta} v_{\theta}}{2}+\frac{\alpha_{t} v_{t}}{2 \alpha}-\frac{\alpha_{\theta}^{2}}{4 \alpha}+\frac{\alpha_{\theta \theta}}{2}-U_{t}^{2}+\alpha U_{\theta}^{2}+\frac{e^{4 U}}{4 t^{2}}\left(A_{t}^{2}-A_{\theta}^{2}\right)-\frac{3 \alpha e^{2 v} K^{2}}{4 t^{4}}+\alpha \Lambda e^{2(v-U),} \\
U_{t t}-\alpha U_{\theta \theta} & =-\frac{U_{t}}{t}+\frac{\alpha_{\theta} U_{\theta}}{2}+\frac{\alpha_{t} U_{t}}{2 \alpha}+\frac{e^{4 U}}{2 t^{2}}\left(A_{t}^{2}-\alpha A_{\theta}^{2}\right)+\alpha \Lambda e^{2(v-U)}, \\
A_{t t}-\alpha A_{\theta \theta} & =\frac{A_{t}}{t}+\frac{\alpha_{\theta} A_{\theta}}{2}+\frac{\alpha_{t} A_{t}}{2 \alpha}-4\left(A_{t} U_{t}-\alpha A_{\theta} U_{\theta}\right) .
\end{aligned}
$$

Auxiliary equations:

$$
\begin{aligned}
& 0=G_{t}+A H_{t}, \\
& 0=H_{t}-\frac{\sqrt{\alpha} e^{2 v} K}{t^{3}} .
\end{aligned}
$$

Note that the Killing fields have been chosen such that the twist quantity $J$ vanishes and note that $K$ is a nonnegative constant (see Section 2A).

Let us define the following replacement for the function $U$ :

$$
P=2 U-\ln t
$$

We refer to the discussion on page 202 for the motivation for the introduction of the quantity $P$.

The evolution equation for $U$ leads to the following equation for $P$ :

$$
P_{t t}-\alpha P_{\theta \theta}=\left(-\frac{1}{t}+\frac{1}{2} \frac{\alpha_{t}}{\alpha}\right) P_{t}+\frac{\alpha_{\theta} P_{\theta}}{2}+e^{2 P}\left(A_{t}^{2}-\alpha A_{\theta}^{2}\right)-\frac{1}{2 t^{4}} \alpha e^{2 v} K^{2} .
$$

As mentioned on page 202, this equation is homogeneous in the Gowdy case $K=0$, since there are no terms containing $\Lambda$ compared to (108). In the following, it will be useful to work both with $P$ and $U$ and to use two energy densities, one associated with the system of wave equations for $(U, A)$ and one associated with the system of wave equations for $(P, A)$.

8B. The universal cover of $M / T^{2}$. In Section $8 \mathrm{~K}$, we will study the characteristic equation which defines null rays in areal coordinates. It will be easier to address this problem in the universal cover of the quotient of the spacetime. For any $T^{2}$-symmetric spacetimes $(\mathcal{M}, g)$, we introduce $2=M / T^{2}$, the quotient of the spacetime by the orbits of symmetry, and then define $\tilde{\mathscr{Q}}$ as the universal cover of 2 . Let $\pi_{1}: M \rightarrow 2$ be the natural projection from $M$ to 2 .

Suppose $(M, g)$ is foliated by areal coordinates with the metric taking the form (2). Let $\alpha_{Q}$ be such that $\alpha$ is the pull-back of $\alpha_{Q}$ by $\pi_{1}^{*}$. We then define $\tilde{\alpha}$ to be the lift to $\tilde{2}$ of $\alpha_{Q}$. We may define similarly 
tilde functions for all metric functions, such as $\tilde{v}, \tilde{U}$, etc. Note that $\tilde{\mathcal{Q}}$ has topology $\mathbb{R} \times \mathbb{R}$ and admits areal coordinates $(\tilde{t}, \tilde{\theta}) \in\left(t_{f} \cdot t_{i}\right] \times \mathbb{R}$ and Lorentzian metric:

$$
d s^{2}=-e^{2(\tilde{v}-\tilde{U})}\left(\tilde{\alpha} d \tilde{t}^{2}-d \tilde{\theta}^{2}\right) .
$$

Note also that all tilde functions $\tilde{v}, \tilde{U}$, etc. are periodic in $\theta$ with period 1 and that they satisfy the system of equations (104)-(111) on $\left(t_{f} \cdot t_{i}\right] \times \mathbb{R}$.

In the following, we will often, ${ }^{18}$ by an abuse of notation, ${ }^{19}$ drop the tildes on functions defined on $\tilde{\mathscr{Q}}$.

8C. The contradiction setting. As explained in Section 6, the proof will follow by contradiction. Let us thus assume that $(M, g)$ is the past maximal development of vacuum $T^{2}$-symmetric spacetimes with $\Lambda>0$ such that $t_{0}>0$. By Proposition 1, there exist a global areal foliation where the metric takes the form (2) and such that $t$ lies in $\left(t_{0}, t_{i}\right]$. Thus, there exists functions $\alpha, v, U, A$ defined on $\left(t_{0}, t_{i}\right] \times[0,1]$ which are periodic in $\theta$ with period 1 , and a constant $K$ such that $\alpha, v, U, A$ and $K$ satisfy the system of equations (104), (109). Moreover, since the cases where $\Lambda=0$ have already been treated, and since the cases where $K=0, \Lambda>0$ may be treated by similar methods as we explained in the previous section, we will suppose that we are in the case where $K>0$ and $\Lambda>0$. Finally, let us assume that the assumptions of Theorem 1 hold, i.e., the spacetime is not polarized.

8D. Uniform blow up of $\alpha$. The contradiction setting immediately implies the following:

Lemma 8.1. Under the assumptions of Section $8 C$, for all $\theta \in[0,1]$, we have $\alpha(t, \theta) \rightarrow \infty$ as $t \rightarrow t_{0}$ and $\min _{\theta \in[0,1]} \alpha(t, \theta) \rightarrow \infty$ as $t \rightarrow t_{0}$.

Proof. Suppose the lemma does not hold. Because $\alpha$ is monotonic, it follows that $\min _{\theta \in[0,1]} \alpha(t, \theta)$ is uniformly bounded, i.e., results similar to those of Section $7 \mathrm{H}$ hold. We may then apply similar estimates as the estimates of sections $7 \mathrm{I}$ to $7 \mathrm{~N}$, replacing $f$ by 0 everywhere. Indeed, the presence of the Vlasov matter was necessary only so as to ensure that the content of Section $7 \mathrm{H}$ is valid. Proposition 2 then applies, and thus $(\mathcal{M}, g)$ is not maximal, a contradiction.

Remark 8D.1. Since the rest of the proof of Theorem 2 will rely on the assumptions of Section 8C, it will be from now on assumed that they hold.

8E. The basic energy estimates. We will need to work with several energy densities and several energy integrals. Let us thus define

$$
\begin{aligned}
g & =U_{t}^{2}+\alpha U_{\theta}^{2}+\frac{e^{4 U}}{4 t^{2}}\left(A_{t}^{2}+\alpha A_{\theta}^{2}\right), \\
h & =P_{t}^{2}+\alpha P_{\theta}^{2}+e^{2 P}\left(A_{t}^{2}+\alpha A_{\theta}^{2}\right) . \\
E_{g}(t) & =\int_{[0,1]} \frac{g}{\sqrt{\alpha}} d \theta, \\
E_{h}(t) & =\int_{[0,1]} \frac{h}{\sqrt{\alpha}} d \theta,
\end{aligned}
$$

\footnotetext{
${ }^{18}$ That is to say, we shall use the same symbol for a function defined on $\mathcal{M}$ and for its associated tilde function.

${ }^{19}$ Note that strictly speaking, in the analysis of Section 7 , all metric functions were also defined on 2 rather than $M$ since we had considered them to be function of $(t, \theta)$. The same remark applies for the analysis carried in Section 9.
} 


$$
\begin{aligned}
E_{h, K}(t) & =E_{h}(t)+\int_{[0,1]} \frac{\sqrt{\alpha} e^{2 v} K^{2}}{t^{4}} d \theta, \\
E_{h, K, \Lambda}(t) & =E_{h}(t)+\int_{[0,1]}\left(\frac{\sqrt{\alpha} e^{2 v} K^{2}}{t^{4}}+4 \Lambda \frac{\sqrt{\alpha} e^{2 v-P}}{t}\right) d \theta .
\end{aligned}
$$

Several computations will also be useful for the rest of the analysis. First, using the constraint equations (104) and (106), we have the identities

$$
\begin{aligned}
\frac{\partial}{\partial t}\left(\frac{\sqrt{\alpha} e^{2 v-P}}{t}\right) & =\frac{1}{2} \sqrt{\alpha} e^{2 v-P}\left(h-\frac{1}{t^{2}}\right), \\
\frac{\partial}{\partial t}\left(\sqrt{\alpha} e^{2 v}\right) & =2 t \sqrt{\alpha} e^{2 v} g .
\end{aligned}
$$

Taking the time derivative of $E_{h}$ and using the Einstein equations, we obtain

$$
\begin{array}{r}
\frac{d E_{h}}{d t}=-\frac{2}{t} \int_{[0,1]} \frac{P_{t}^{2}}{\sqrt{\alpha}}+e^{2 P} \sqrt{\alpha} A_{t}^{2}-2 \Lambda \int_{[0,1]} \sqrt{\alpha} e^{2 v-P} h-\frac{2}{t^{3}} \int_{[0,1]} \sqrt{\alpha} e^{2 v} K^{2} g+\frac{1}{2 t^{5}} \int_{[0,1]} \sqrt{\alpha} e^{2 v} K^{2} \\
-\frac{2}{t} \int_{[0,1]} \sqrt{\alpha} P_{\theta \theta}+\frac{\alpha_{\theta}}{2 \sqrt{\alpha}} P_{\theta} .
\end{array}
$$

The terms on the last line vanish thanks to the $\theta$ periodicity, so we obtain ${ }^{20}$

$$
\frac{d E_{h}}{d t}=-\frac{2}{t} \int_{[0,1]} \frac{P_{t}^{2}}{\sqrt{\alpha}}+\frac{e^{2 P}}{\sqrt{\alpha}} A_{t}^{2}-2 \Lambda \int_{[0,1]} \sqrt{\alpha} e^{2 v-P} h-\frac{2}{t^{3}} \int_{[0,1]} \sqrt{\alpha} e^{2 v} K^{2} g+\frac{1}{2 t^{5}} \int_{[0,1]} \sqrt{\alpha} e^{2 v} K^{2} .
$$

or, written only in terms of $h$ and $P_{t}$,

$$
\frac{d E_{h}}{d t}=-\frac{2}{t} \int_{[0,1]} \frac{P_{t}^{2}}{\sqrt{\alpha}}+\frac{e^{2 P}}{\sqrt{\alpha}} A_{t}^{2}-\int_{[0,1]} \frac{P_{t}}{\sqrt{\alpha} t^{4}} \alpha e^{2 v} K^{2}+\int_{[0,1]} \frac{1}{2} \frac{\alpha_{t}}{\alpha^{3 / 2}} h .
$$

We see that the last term on the right-hand side of (124) is competing against the others.

Remark 8E.1. In the case where $K=0$, the last term vanishes, thus, we obtain the desired monotonicity $^{21}$ on $E_{h}$ and we could conclude as in [Isenberg and Weaver 2003]. Thus, we obtain:

Proposition 4. Let $(M, g)$ be the maximal development of $T^{2}$-symmetric initial data in the vacuum with $\Lambda \geq 0$ and $K=0$. Suppose that $E_{h}$ does not vanish identically. Then $(M, g)$ admits a global foliation by areal coordinates with the time coordinate $t$ taking all values in $(0, \infty)$, i.e., $t_{0}=0$ in the notation of Proposition 1.

Unfortunately, in the general case, we lose this monotonicity and the analysis is, as we will see, more complex.

\footnotetext{
${ }^{20}$ That the terms involving derivatives in $\theta$ add up to an exact derivative is due to the wave map background structure of the equations. See [Berger et al. 1997].

${ }^{21}$ Note that the parallelism between the cases $(K>0, \Lambda=0)$ and $(K=0, \Lambda>0)$ does not extend beyond the issue of the value of $t_{0}$. Indeed, once we know that $t_{0}=0$, the different powers of $t$ for the terms containing $\Lambda$ and $K$ in (106) are likely to yield different asymptotics for the solutions.
} 
We may also compute the time derivative of $E_{h, K}$ and $E_{h, K, \Lambda}$ :

$$
\begin{aligned}
\frac{d E_{h, K}}{d t} & =-\frac{2}{t} \int_{[0,1]} \frac{P_{t}^{2}}{\sqrt{\alpha}}+e^{2 P} \sqrt{\alpha} A_{t}^{2}-2 \Lambda \int_{[0,1]} \sqrt{\alpha} e^{2 v-P} h-\frac{7}{2 t^{5}} \int_{[0,1]} \sqrt{\alpha} e^{2 v} K^{2}, \\
\frac{d E_{h, K, \Lambda}}{d t} & =-\frac{2}{t} \int_{S^{1}[0,1]} \frac{P_{t}^{2}}{\sqrt{\alpha}}+e^{2 P} \sqrt{\alpha} A_{t}^{2}-\frac{7}{2 t^{5}} \int_{[0,1]} \sqrt{\alpha} e^{2 v} K^{2}-2 \Lambda \int_{[0,1]} \frac{\sqrt{\alpha} e^{2 v-P}}{t^{2}} .
\end{aligned}
$$

We see in particular that $E_{h, K}$ and $E_{h, K, \Lambda}$ are nondecreasing with decreasing time. ${ }^{22}$

Lemma 8.2. $E_{g}, E_{h}, E_{h, K}$ and $E_{h, K, \Lambda}$ are uniformly bounded on $\left(t_{0}, t_{i}\right]$ and the last two quantities can be continuously extended to $t_{0}$.

Proof. From (127), we have

$$
\frac{d E_{h, K, \Lambda}}{d t} \geq-\frac{7}{2 t} E_{h, K, \Lambda}
$$

By application of Gronwall's lemma, therefore, $E_{h, K, \Lambda}$ is bounded uniformly if $t_{0}>0$. However, since

$$
E_{h} \leq E_{h, K} \leq E_{h, K, \Lambda},
$$

we also obtain a uniform bound on $E_{h}$ and $E_{h, K}$. Since $E_{h, K}$ and $E_{h, K, \Lambda}$ are monotonically increasing they admit strictly positive limits at $t=t_{0}$. A similar analysis implies the uniform bound on $E_{g}$.

8F. Continuous extensions of the twist and cosmological energies. In order to extract some information from the continuous extensions of $E_{h, K}$ and $E_{h, K, \Lambda}$, we will need the following:

Lemma 8.3. The functions $\sqrt{\alpha} e^{2 v}$ and $\sqrt{\alpha} e^{2 v-P}$, and therefore also $\sqrt{\alpha} e^{2 v} K^{2} / t^{4}$ and $\Lambda \sqrt{\alpha} e^{2 v-P} / t$, admit continuous extensions to $t=t_{0}$ and are uniformly bounded in $\left(t_{0}, t_{i}\right] \times[0,1]$.

Proof. The derivatives with respect to $t$ of $\sqrt{\alpha} e^{2 v}$ and $t^{-1 / 2} \sqrt{\alpha} e^{2 v-P}$ are positive, as can be verified by direction computation. Therefore, they are monotonically decreasing in the past direction and admit continuous extensions to $t=t_{0}$. Moreover, they are bounded by the maximum of their values on the initial data surface, which is finite by compactness.

Since $\sqrt{\alpha} e^{2 v}$ and $t^{-1 / 2} \sqrt{\alpha} e^{2 v-P}$ are pointwise decreasing with $t$ in the past direction and are positive, their integrals over $\theta$ at fix $t$ are positive functions which are decreasing in the past direction and therefore, they admit a limit as $t$ goes to $t_{0}$. Thus:

Lemma 8.4. The integrals

$$
\int_{[0,1]} \frac{\sqrt{\alpha} e^{2 v} K^{2}}{t^{4}} d \theta \text { and } \int_{[0,1]} \Lambda \frac{\sqrt{\alpha} e^{2 v-P}}{t} d \theta
$$

admit continuous extensions to $t=t_{0}$.

8G. Estimate for the spatial derivatives of $\beta$ and $\beta-P / 2$. We define $\beta$ as in the Vlasov case by

$$
e^{2 \beta}=\alpha e^{2 v} .
$$

${ }^{22}$ Note that this monotonicity cannot be used as a replacement of the monotonicity of $E_{g}$ or $E_{h}$, since no estimate similar to (13) can hold when $E_{g}$ is replaced by $E_{h, K}$ or $E_{h, K, \Lambda}$, as can be seen by studying homogeneous plane symmetric solutions. 
It follows as in Lemma 7.5 that $\beta_{\theta}$ is bounded by $g / \sqrt{\alpha}$ :

$$
\left|\beta_{\theta}\right| \leq t \frac{g}{\sqrt{\alpha}}
$$

By integration, we obtain:

Lemma 8.5. For all $t \in\left(t_{0}, t_{i}\right]$,

$$
\max _{[0,1]} \beta(t, \cdot)-\min _{[0,1]} \beta(t, \cdot) \leq t_{i} E_{g} .
$$

In particular, $\max _{[0,1]} \beta(t, \cdot)-\min _{[0,1]} \beta(t, \cdot)$ is uniformly bounded.

We may do the same analysis using $h$ and $P$. First, we rewrite (105) as

$$
\beta_{\theta}-\frac{P_{\theta}}{2}=\frac{t}{2}\left(P_{t} P_{\theta}+e^{2 P} A_{t} A_{\theta}\right)
$$

from which we obtain that

$$
\left|\beta_{\theta}-\frac{P_{\theta}}{2}\right| \leq \frac{t}{4} \frac{h}{\sqrt{\alpha}}
$$

Therefore, using the bounds on $E_{h}$, we have:

Lemma 8.6. For all $t \in\left(t_{0}, t_{i}\right]$,

$$
\max _{[0,1]}(2 \beta-P)(t, \cdot)-\min _{[0,1]}(2 \beta-P)(t, \cdot) \leq \frac{t_{i}}{2} E_{h} .
$$

In particular, $\max _{[0,1]}(2 \beta-P)(t, \cdot)-\min _{[0,1]}(2 \beta-P)(t, \cdot)$ is uniformly bounded.

\section{H. Limit of the gravitational energy of the orbits of symmetry.}

Lemma 8.7. For all $\epsilon>0$ there exists $t_{\epsilon}>t_{0}$ such that either $E_{h}\left(t_{\epsilon}\right) \leq \epsilon$ or $E_{g}\left(t_{\epsilon}\right) \leq \epsilon$.

Proof. Suppose the lemma does not hold. Then there exists an $\epsilon>0$ such that $\min \left(E_{h}, E_{g}\right)>\epsilon$ for all $t>t_{0}$.

By integration of (124), we have, for all $t \in\left(t_{0}, t_{i}\right]$,

$\int_{t}^{t_{i}} 2 \Lambda \int_{[0,1]} \sqrt{\alpha} e^{2 v-P} h d \theta d t^{\prime}+\int_{t}^{t_{i}} \frac{2 K^{2}}{t^{3}} \sqrt{\alpha} e^{2 v} g d \theta d t^{\prime} \leq E_{h}(t)-E_{h}\left(t_{i}\right)+\int_{t}^{t_{i}} \frac{1}{2 t^{5}} \int_{[0,1]} \sqrt{\alpha} e^{2 v} K^{2}$.

Since all terms on the right-hand side are bounded by Lemmas 8.2 and 8.4, we have in particular, that, there exits some constant $D>0$ such that

$$
\int_{t}^{t_{i}} 2 \Lambda \int_{[0,1]} \sqrt{\alpha} e^{2 v-P} h d \theta d t \leq D .
$$

Using the control on the spatial derivatives of $2 \beta-P$ obtained in Lemma 8.6, we obtain, for all $(t, \theta)$ in $\left(t_{0}, t_{i}\right] \times[0,1]$,

$$
\begin{array}{r}
\int_{t}^{t_{i}} \int_{[0,1]} e^{2 \beta-P} \frac{h}{\sqrt{\alpha}} d \theta d s \leq B, \\
\int_{t}^{t_{i}} \min _{\theta^{\prime} \in[0,1]} e^{2 \beta-P}(s, \cdot) E_{h}(s) d s \leq B,
\end{array}
$$




$$
\begin{aligned}
\int_{t}^{t_{i}} \min _{\theta^{\prime} \in[0,1]} e^{2 \beta-P}(s, \cdot) d s \leq \frac{B}{\epsilon}, \\
\int_{t}^{t_{i}} e^{2 \beta-P}(s, \theta) d s \leq \frac{B}{\epsilon}+B^{\prime}\left(t_{i}-t\right), \\
\int_{t}^{t_{i}} e^{2 \beta-P}(s, \theta) d s \leq B^{\prime \prime},
\end{aligned}
$$

for some constants $B>0, B^{\prime}>0$ and $B^{\prime \prime}>0$.

Similarly, one obtains from inequality (136) and Lemma 8.5 the existence of a constant $B^{\prime \prime \prime}>0$ such that, for all $(t, \theta) \in\left(t_{0}, t_{i}\right]$

$$
\int_{t}^{t_{i}} e^{2 \beta}(s, \theta) d s \leq B^{\prime \prime \prime} .
$$

It follows from (142) and (143) that the right-hand side of (106) is bounded and by integration, $\ln \alpha$ and therefore $\alpha$ are uniformly bounded above, which contradicts Lemma 8.1.

We may now prove a stronger version of Lemma 8.7:

Lemma 8.8. $E_{h} \rightarrow 0$ as $t \rightarrow 0$ and $E_{g} \rightarrow 0$ as $t \rightarrow 0$.

Proof. We have $E_{h}=E_{h, K}-\int_{[0,1]}\left(\sqrt{\alpha} e^{2 v} K^{2} / t^{4}\right) d \theta$. In view of Lemmas 8.2 and 8.4, both terms on the right-hand side have a limit, thus $E_{h}$ has a limit. Similarly, $E_{g}$ has a limit. In view of the last lemma, both limits cannot be strictly positive and therefore at least one of them has to be zero. Suppose for instance, that $E_{h}$ tends to 0 as $t$ tends to $t_{0}$. From the definition of $h, g, P$ and $U$ it follows that

and therefore

$$
g=\frac{h}{4}+\frac{P_{t}}{2 t}+\frac{1}{4 t^{2}}
$$

$$
g \leq \frac{h}{2}+\frac{1}{2 t^{2}}
$$

Since on the other hand, $\sqrt{\alpha}$ tends to infinity uniformly in $\theta$ by Lemma 8.1 , it follows from the last inequality that $E_{g}$ also tends to 0 as $t$ tends to $t_{0}$. The case where we know a priori that $E_{g}$ tends to 0 and we need to deduce that $E_{h}$ tends to 0 may be treated similarly.

8I. Strong control on the spatial derivative of $\boldsymbol{\beta}$. An immediate application of these limits allows an improvement to Lemmas 8.5 and 8.6:

Lemma 8.9.

$$
\begin{array}{r}
\lim _{t \rightarrow t_{0}}\left(\max _{[0,1]} \beta(t, \cdot)-\min _{[0,1]} \beta(t, \cdot)\right)=0, \\
\lim _{t \rightarrow t_{0}}\left(\max _{[0,1]}(2 \beta-P)(t, \cdot)-\min _{[0,1]}(2 \beta-P)(t, \cdot)\right)=0 .
\end{array}
$$

From this it follows that:

Lemma 8.10. For all $\epsilon>0$, there exists $t^{\prime}>t_{0}$ such that, for all $t \in\left(t_{0}, t^{\prime}\right]$,

$$
\begin{gathered}
\max _{\theta \in[0,1]} e^{2 \beta}(t, \cdot) \leq e^{\epsilon} \min _{[0,1]} e^{2 \beta}(t, \cdot), \\
\max _{\theta \in[0,1]} e^{2 \beta-P}(t, \cdot) \leq e^{\epsilon} \min _{[0,1]} e^{2 \beta-P}(t, \cdot), \\
\max _{\theta \in[0,1]}\left(-\frac{\alpha_{t}}{\alpha}(t, \cdot)\right) \leq e^{\epsilon} \min _{\theta \in[0,1]}\left(-\frac{\alpha_{t}}{\alpha}(t, \cdot)\right) .
\end{gathered}
$$


Proof. The first two inequalities follow directly from the last lemma. Next, write the Einstein equation for $\alpha,(106)$, in terms of $\beta$ and $P$ :

$$
\frac{\alpha_{t}}{\alpha}=-4 e^{2 \beta-P} \Lambda-\frac{e^{2 \beta} K^{2}}{t^{3}} .
$$

Now the last inequality of the lemma follows from the first two.

Note that by integration, we could easily obtain from the last line that for all $\epsilon>0$, there exists $t^{\prime}>t_{0}$ and a constant $C>0$ such that

$$
\max _{\theta \in[0,1]} \alpha(t, \cdot) \leq C \min _{\theta \in[0,1]} \alpha(t, \cdot)^{1+\epsilon} \text { for all } t \in\left(t_{0}, t^{\prime}\right]
$$

Unfortunately, the exponent of the right-hand side is not 1 and this will not be sufficient for our analysis. Thus, we need a stronger estimate than this one, which we provide in the next section.

8J. An estimate for $(\partial / \partial \theta)(\ln \alpha)$. The estimates on $\beta_{\theta}$ and $2 \beta_{\theta}-P_{\theta}$ coming from the inequalities (131) and (134) were based on previously known estimates for $T^{2}$-symmetric spacetimes written in areal coordinates. Here, we will derive a stronger estimate from these inequalities, using the identities (122) and (121) and Equation (106). The estimate that we obtain is the following:

Lemma 8.11. There exists a constant $C>0$ such that, for all $(t, \theta) \in\left(t_{0}, t_{i}\right] \times[0,1]$,

$$
\left|\frac{\partial}{\partial \theta}(\ln (\alpha))(t, \theta)\right| \leq C \text {. }
$$

Proof. Multiplying (131) and (134) by $e^{2 \beta}$ and $e^{2 \beta-P}$, we obtain

$$
\begin{gathered}
\left|\beta_{\theta}\right| e^{2 \beta} \leq t \frac{g}{\sqrt{\alpha}} e^{2 \beta}=\frac{1}{2} \partial_{t}\left(\sqrt{\alpha} e^{2 v}\right), \\
\left|\beta_{\theta}-\frac{P_{\theta}}{2}\right| e^{2 \beta-P} \leq \frac{t}{4} \frac{h}{\sqrt{\alpha}} e^{2 \beta-P}=\frac{t^{1 / 2}}{2} \partial_{t}\left(t^{-1 / 2} \sqrt{\alpha} e^{2 v-P}\right),
\end{gathered}
$$

where we have used the identities (122) and (121) arising from the constraints to rewrite the right-hand sides of the equations.

On the other end, from (106), we have

$$
-\frac{\partial}{\partial t} \ln \alpha=4 \Lambda e^{2 \beta-P}+\frac{K^{2} e^{2 \beta}}{t^{3}} .
$$

Thus, taking the $\theta$ derivative of the last equation, we obtain

$$
-\frac{\partial}{\partial \theta}\left(\frac{\partial}{\partial t} \ln \alpha\right)=4 \Lambda\left(2 \beta_{\theta}-P_{\theta}\right) e^{2 \beta-P}+2 \beta_{\theta} \frac{K^{2} e^{2 \beta}}{t^{3}} .
$$

We now integrate the last line and commute the $\theta$ and $t$ partial derivatives in the integrand of the left-hand side to obtain, for all $(t, \theta) \in\left(t_{0}, t_{i}\right] \times[0,1]$,

$$
\partial_{\theta} \ln \alpha(t, \theta)=\partial_{\theta} \ln \alpha\left(t_{i}, \theta\right)+\int_{t}^{t_{i}} 4 \Lambda\left(2 \beta_{\theta}-P_{\theta}\right) e^{2 \beta-P}(s, \theta) d s+\int_{t}^{t_{i}} 2 \beta_{\theta} \frac{K^{2} e^{2 \beta}}{t^{3}}(s, \theta) d s .
$$


Using (152) and (153), we have

$$
\begin{aligned}
\left|\partial_{\theta} \ln \alpha(t, \theta)\right| & \leq \sup _{\theta \in[0,1]}\left|\partial_{\theta} \ln \alpha\left(t_{i}, \cdot\right)\right|+\int_{t}^{t_{i}} 4 \Lambda\left|\left(2 \beta_{\theta}-P_{\theta}\right) e^{2 \beta-P}\right|(s, \theta) d s+\int_{t}^{t_{i}}\left|2 \beta_{\theta} \frac{K^{2} e^{2 \beta}}{t^{3}}\right|(s, \theta) d s, \\
& \leq \sup _{\theta \in[0,1]}\left|\partial_{\theta} \ln \alpha\left(t_{i}, \cdot\right)\right|+4 \Lambda t_{i}^{1 / 2} \int_{t}^{t_{i}} \partial_{t}\left(t^{-1 / 2} \sqrt{\alpha} e^{2 v-P}\right)+\frac{K^{2}}{t_{0}^{3}} \int_{t}^{t_{i}} \partial_{t}\left(\sqrt{\alpha} e^{2 v}\right)
\end{aligned}
$$

and the lemma follows from the uniform bounds on $\sqrt{\alpha} e^{2 v}$ and $\sqrt{\alpha} e^{2 v-P}$.

By integration, we immediately obtain:

Corollary 2. There exists a constant $C>0$ such that, for all $t \in\left(t_{0}, t_{i}\right]$, we have

$$
\max _{\theta \in[0,1]} \alpha(t, \theta) \leq C \min _{\theta \in[0,1]} \alpha(t, \theta) .
$$

Combining this with Lemma 8.5, we may obtain:

Corollary 3. There exist constants $M_{1}$ and $M_{2}$ such that for all $(t, \theta) \in\left(t_{0}, t_{i}\right]$, we have

$$
M_{1} \sqrt{\alpha}(t, \theta) \geq e^{2 \beta}(t, \theta) \geq M_{2} \sqrt{\alpha}(t, \theta) .
$$

Similarly, there exist constants $M_{1}^{\prime}$ and $M_{2}^{\prime}$ such that for all for all $(t, \theta) \in\left(t_{0}, t_{i}\right]$, we have

$$
M_{1}^{\prime} \sqrt{\alpha}(t, \theta) \geq e^{2 \beta-P}(t, \theta) \geq M_{2}^{\prime} \sqrt{\alpha}(t, \theta) .
$$

Proof. Given that $E_{h, K}$ is nondecreasing in the past direction, that $E_{h}$ tends to zero as $t$ tends to $t_{0}$ and that $K>0$, it follows that the limit of $\int_{[0,1]} \sqrt{\alpha} e^{2 v} d \theta$ is nonzero. This implies, using the monotonicity of $\sqrt{\alpha} e^{2 v}$ as a function of $t$ and the monotone convergence theorem, that there exists a $\theta_{0}$ and a constant $M>0$ such that $\sqrt{\alpha} e^{2 v}\left(t, \theta_{0}\right) \geq M$ for all $t \in\left(t_{0}, t_{i}\right]$. Let $M^{\prime}$ be an upper bound for $\sqrt{\alpha} e^{2 v}\left(t, \theta_{0}\right)$. By Lemma 8.5, there exits a constant $M^{\prime \prime}$ such that, for all $(t, \theta) \in\left(t_{0}, t_{i}\right] \times[0,1]$,

$$
e^{M^{\prime \prime}} e^{2 \beta}\left(t, \theta_{0}\right) \geq e^{2 \beta}(t, \theta) \geq e^{-M^{\prime \prime}} e^{2 \beta}\left(t, \theta_{0}\right)
$$

and thus

$$
M^{\prime} e^{M^{\prime \prime}} \sqrt{\alpha}\left(t, \theta_{0}\right) \geq e^{2 \beta}(t, \theta) \geq M e^{-M^{\prime \prime}} \sqrt{\alpha}\left(t, \theta_{0}\right)
$$

Let $M^{\prime \prime \prime}$ be such that, for all $(t, \theta) \in t \in\left(t_{i}, t_{0}\right] \times[0,1]$,

$$
e^{M^{\prime \prime \prime}} \sqrt{\alpha}(t, \theta) \geq \sqrt{\alpha}\left(t, \theta_{0}\right) \geq e^{-M^{\prime \prime \prime}} \sqrt{\alpha}(t, \theta) .
$$

Then we have

$$
M^{\prime} e^{M^{\prime \prime \prime}} e^{M^{\prime \prime}} \sqrt{\alpha}(t, \theta) \geq e^{2 \beta}(t, \theta) \geq M e^{-M^{\prime \prime \prime}} e^{-M^{\prime \prime}} \sqrt{\alpha}(t, \theta)
$$

This proves the inequalities (159). The second set of inequalities can be treated similarly, using $E_{g}$ and another energy integral

$$
E_{g, \Lambda}=\int_{[0,1]}\left(\frac{g}{\sqrt{\alpha}}+\alpha e^{2(\nu-U)} \Lambda\right) d \theta,
$$

which may be easily proven to be nondecreasing in the past direction and uniformly bounded. 
The aim of the next two sections will be to describe the characteristics curves and to establish several estimates about their behavior for $t$ close to $t_{0}$. We will actually not need to analyze all null curves, but only null curves orthogonal to the orbits of symmetry. Note that in the next sections, we will often, by an abuse of notation, denote by the same name functions defined on $\mathcal{M}$ or 2 together with their lifts to $\tilde{2}$, the universal cover of 2 .

8K. An analysis of the characteristics in areal coordinates. Consider a null curve $\gamma$ in $M$ which is orthogonal to the orbits of symmetry and let $\tilde{\gamma}$ be the lift to $\tilde{2}$ of the projection to 2 of $\gamma$. In null coordinates as those used in [Smulevici 2008], $\gamma$ is given by $u=$ constant or $v=$ constant. In areal coordinates, we obtain $\gamma$ by solving the characteristic equation

$$
\Theta^{\prime}(s)= \pm \sqrt{\alpha(s, \Theta(s))},
$$

with appropriate initial conditions. If $\Theta(t)$ is a solution to the above equation, then $\gamma$ is given in areal coordinates by $(t, \Theta(t))$.

By standard arguments, solutions of (166) exist and are smooth and unique on $\left(t_{0}, t\right]$ for any $t \in\left(t_{0}, t_{i}\right]$ once initial conditions have been fixed.

Now let us consider the characteristics parallel to the constant $v$ lines. They are parametrized by $(s, \Theta(s, \theta, t))$, where $\Theta(s, \theta, t)$ satisfies

$$
\Theta(s, \theta, t)=\theta-\int_{t}^{s} \sqrt{\alpha\left(s^{\prime}, \Theta\left(s^{\prime}, \theta, t\right)\right)} d s^{\prime} .
$$

Taking the $\theta$ derivative,

$$
\Theta_{\theta}(s, \theta, t)=1-\int_{t}^{s} \frac{1}{2}\left(\frac{\alpha_{\theta}}{\sqrt{\alpha}}\right)\left(s^{\prime}, \Theta\left(s^{\prime}, \theta, t\right)\right) \Theta_{\theta}\left(s^{\prime}, \theta, t\right) d s^{\prime} .
$$

Solving this equation implicitly, we see that

$$
\Theta_{\theta}(s, \theta, t)=\exp \int_{s}^{t} \frac{1}{2}\left(\frac{\alpha_{\theta}}{\sqrt{\alpha}}\right)\left(s^{\prime}, \Theta(s, \theta, t)\right) d s^{\prime} .
$$

We are naturally lead to estimate the integral on the right. This is the subject of the next section.

\section{L. Estimates for the integral along the characteristics of $\alpha_{\theta} / \sqrt{\alpha}$.}

Lemma 8.12. For all $\epsilon>0$, there exists a $\bar{t}>t_{0}$, such that for all $t^{\prime} \in\left(t_{0}, \bar{t}\right]$ there exists a negative constant $M_{1}$ and a positive constant $M_{2}$ such that, for all $(t, \theta) \in\left(t_{0}, t^{\prime}\right] \times[0,1]$,

$$
M_{1}-\epsilon \ln \alpha\left(t, \Theta\left(t, \theta, t^{\prime}\right)\right) \leq \int_{t}^{t^{\prime}}-\frac{\alpha_{\theta}}{\sqrt{\alpha}}\left(s, \Theta\left(s, \theta, t^{\prime}\right)\right) d s \leq M_{2}+\epsilon \ln \alpha\left(t, \Theta\left(t, \theta, t^{\prime}\right)\right) \text {. }
$$

Proof. Let $\epsilon>0$ and let $\bar{t} \in\left(t_{0}, t_{i}\right]$ be such that Lemma 8.10 holds in the following way: for all $\left(t, \theta, \theta^{\prime}\right) \in\left(t_{0}, \bar{t}\right] \times[0,1]^{2}$,

$$
-(1-\epsilon) \frac{\alpha_{t}}{\alpha}\left(t, \theta^{\prime}\right)<-\frac{\alpha_{t}}{\alpha}(t, \theta)<-(1+\epsilon) \frac{\alpha_{t}}{\alpha}\left(t, \theta^{\prime}\right) .
$$


Fix $t^{\prime} \in\left(t_{0}, \bar{t}\right]$ and let $\Theta\left(t, \theta, t^{\prime}\right)$ be a characteristic such that

$$
\Theta\left(t, \theta, t^{\prime}\right)=\theta-\int_{t^{\prime}}^{t} \sqrt{\alpha}\left(s, \Theta\left(s, \theta, t^{\prime}\right)\right) d s
$$

We have, for all $(t, \theta) \in\left(t_{0}, t^{\prime}\right] \times[0,1]$,

$$
\begin{aligned}
\int_{t}^{t^{\prime}}-\frac{\alpha_{\theta}}{\sqrt{\alpha}}\left(s, \Theta\left(s, \theta, t^{\prime}\right)\right) d s & =\int_{t}^{t^{\prime}}\left(\frac{\alpha_{t}}{\alpha}-\frac{\alpha_{\theta}}{\sqrt{\alpha}}-\frac{\alpha_{t}}{\alpha}\right)\left(s, \Theta\left(s, \theta, t^{\prime}\right)\right) d s \\
& =\int_{t}^{t^{\prime}} \frac{d}{d s}\left(\ln \alpha\left(s, \Theta\left(s, \theta, t^{\prime}\right)\right) d s-\int_{t}^{t^{\prime}} \frac{\alpha_{t}}{\alpha}\left(s, \Theta\left(s, \theta, t^{\prime}\right)\right) d s .\right.
\end{aligned}
$$

We now use (171) to estimate the second integral on the right-hand side. Let $\theta_{0}$ be in $[0,1]$. Then

$$
\begin{aligned}
& -\int_{t}^{t^{\prime}} \frac{\alpha_{t}}{\alpha}\left(s, \Theta\left(s, \theta, t^{\prime}\right)\right) d s \geq-(1-\epsilon) \int_{t}^{t^{\prime}} \frac{\alpha_{t}}{\alpha}\left(s, \theta_{0}\right) d s, \\
& -\int_{t}^{t^{\prime}} \frac{\alpha_{t}}{\alpha}\left(s, \Theta\left(s, \theta, t^{\prime}\right)\right) d s \geq-(1-\epsilon)\left(\ln \alpha\left(t^{\prime}, \theta_{0}\right)-\ln \left(\alpha\left(t, \theta_{0}\right)\right)\right) .
\end{aligned}
$$

Using Corollary 2, there exists a constant $M>0$ such that

$$
-\int_{t}^{t^{\prime}} \frac{\alpha_{t}}{\alpha}\left(s, \Theta\left(s, \theta, t^{\prime}\right)\right) d s \geq-(1-\epsilon)\left(\ln \alpha\left(t^{\prime}, \Theta\left(t^{\prime}, \theta, t^{\prime}\right)\right)-\ln \alpha\left(t, \Theta\left(t, \theta, t^{\prime}\right)\right)\right)-M .
$$

Similarly, we obtain

$$
-\int_{t}^{t^{\prime}} \frac{\alpha_{t}}{\alpha}\left(s, \Theta\left(s, \theta, t^{\prime}\right)\right) d s \leq-(1+\epsilon)\left(\ln \alpha\left(t^{\prime}, \Theta\left(t^{\prime}, \theta, t^{\prime}\right)\right)-\ln \alpha\left(t, \Theta\left(t, \theta, t^{\prime}\right)\right)\right)+M .
$$

Thus we have, from (174) and (177):

$\ln \alpha\left(t^{\prime}, \Theta\left(t^{\prime}, \theta, t^{\prime}\right)\right)-\ln \alpha\left(t, \Theta\left(t, \theta, t^{\prime}\right)\right)-(1-\epsilon)\left(\ln \alpha\left(t^{\prime}, \Theta\left(t^{\prime}, \theta, t^{\prime}\right)\right)-\ln \alpha\left(t, \Theta\left(t, \theta, t^{\prime}\right)\right)\right)-M$

$$
\leq \int_{t}^{t^{\prime}}-\frac{\alpha_{\theta}}{\sqrt{\alpha}}\left(s, \Theta\left(s, \theta, t^{\prime}\right)\right) d s
$$

and similarly

$$
\begin{array}{r}
\int_{t}^{t^{\prime}}-\frac{\alpha_{\theta}}{\sqrt{\alpha}}\left(s, \Theta\left(s, \theta, t^{\prime}\right)\right) d s \leq \ln \alpha\left(t^{\prime}, \Theta\left(t^{\prime}, \theta, t^{\prime}\right)\right)-\ln \alpha\left(t, \Theta\left(t, \theta, t^{\prime}\right)\right) \\
-(1+\epsilon)\left(\ln \alpha\left(t^{\prime}, \Theta\left(t^{\prime}, \theta, t^{\prime}\right)\right)-\ln \alpha\left(t, \Theta\left(t, \theta, t^{\prime}\right)\right)\right)+M
\end{array}
$$

The lemma follows by simplifying the terms containing $\alpha(t, \Theta(t, \theta))$ in (179) and (180).

8M. Estimates for the integrals of small powers of $\alpha$. It will be useful for the derivation of pointwise energy estimates to have some control over the integral of $\alpha^{p}$ for small enough $p$. We first need the following result:

Lemma 8.13. There exists $\theta \in[0,1]$, such that

$$
\lim _{t \rightarrow t_{0}} \sqrt{\alpha} e^{2 v}(t, \theta)>0 .
$$


Proof. Suppose that the lemma does not hold. Since $\sqrt{\alpha} e^{2 v}(t, \theta)$ is a decreasing function of $t$, it must then tend to 0 as $t$ tends to $t_{0}$ for any $\theta$. From the compactness of $[0,1]$ and using again the fact that $\sqrt{\alpha} e^{2 v}$ is decreasing in $t$, it follows that $\int_{[0,1]} \sqrt{\alpha} e^{2 v} d \theta$ tends to 0 as $t$ tends to $t_{0}$. This contradicts the facts that $E_{h, K}$ tends to a strictly positive value by monotonicity and $E_{h}$ has limit 0 .

Lemma 8.14. For all $p<\frac{1}{2}$, there exists a function $B\left(t^{\prime}\right)$ such that $B\left(t^{\prime}\right) \rightarrow 0$ as $t^{\prime} \rightarrow t_{0}$ and such that for all $(t, \theta) \in\left(t_{0}, t^{\prime}\right] \times[0,1]$, with $t^{\prime}>t_{0}$, we have

$$
\int_{t}^{t^{\prime}} \alpha^{p}(s, \theta) d s \leq B\left(t^{\prime}\right)
$$

Proof. Let $\theta_{0} \in[0,1]$ be such that the previous lemma holds, and thus such that $\sqrt{\alpha} e^{2 v}\left(\cdot, \theta_{0}\right)$ is bounded from below by a strictly positive constant on $\left(t_{0}, t^{\prime}\right]$.

We then rewrite (106) as

$$
-\left(\frac{1}{2}-p\right) \frac{\alpha_{t}}{\alpha^{3 / 2-p}}=\left(\frac{1}{2}-p\right) \alpha^{p} f\left(t, \theta_{0}\right),
$$

where

$$
f\left(t, \theta_{0}\right)=4 \Lambda \sqrt{\alpha} e^{2 v-P}+\frac{\sqrt{\alpha} e^{2 v} K^{2}}{t^{3}}
$$

is a function bounded from below by a strictly positive constant. Integrating (183), we obtain

$$
\int_{t}^{t^{\prime}} \frac{d}{d t}\left(\alpha^{p-1 / 2}\right) d s=\int_{t}^{t^{\prime}}\left(\frac{1}{2}-p\right) \alpha^{p} f\left(s, \theta_{0}\right) d s .
$$

Using the lower bound on $f(s, \theta)$, we therefore obtain

$$
\int_{t}^{t^{\prime}} \alpha^{p}\left(s, \theta_{0}\right) d s \leq \frac{C}{\alpha^{1 / 2-p}\left(t^{\prime}, \theta_{0}\right)},
$$

for some constant $C>0$. The lemma then follows by application of Corollary 2 of Section 8J and the fact that $\lim _{t^{\prime} \rightarrow t_{0}} \alpha\left(t^{\prime}, \theta_{0}\right)=+\infty$.

From (124), we have seen that $E_{h}$ is a priori not monotonic. In the next section, we will analyze an energy integral associated with the polarization function $A$. The advantage of this energy integral over $E_{h}$ is that, as the wave equation for $A$ is homogeneous, we will be able to extract useful information from the sign of $d E_{A} / d t$.

8N. Analysis of the polarization energy. Define the energy associated with the wave equation for $A$ as

$$
E_{A}=\int_{[0,1]} \frac{e^{2 P}}{\sqrt{\alpha}}\left(A_{t}^{2}+\alpha A_{\theta}^{2}\right) d \theta .
$$

Since by definition $E_{A} \leq E_{h}$, we immediately obtain that $E_{A} \rightarrow 0$, when $t \rightarrow t_{0}$. The aim of this section is to extract some information from this remark. Note that the wave equation for $A,(109)$, may also be written as

$$
\partial_{t}\left(\frac{t e^{2 P} A_{t}}{\sqrt{\alpha}}\right)-\partial_{\theta}\left(t e^{2 P} \sqrt{\alpha} A_{\theta}\right)=0 .
$$


We first compute the time derivative of $E_{A}$ :

$$
\begin{aligned}
\frac{d E_{A}}{d t}= & \int_{[0,1]} \frac{\partial}{\partial t}\left(\frac{e^{2 P}}{\sqrt{\alpha}} A_{t}^{2}\right)+\frac{\partial}{\partial t}\left(e^{2 P} \sqrt{\alpha} A_{\theta}^{2}\right) \\
= & \int_{[0,1]} A_{t} \partial_{t}\left(\frac{e^{2 P}}{\sqrt{\alpha}} A_{t}\right)+A_{t t} \frac{e^{2 P}}{\sqrt{\alpha}} A_{t}+A_{\theta}^{2} \partial_{t}\left(e^{2 P} \sqrt{\alpha}\right)+2 A_{\theta t} A_{\theta} e^{2 P} \sqrt{\alpha} \\
= & \int_{[0,1]} A_{t}\left(\partial_{\theta}\left(e^{2 P} \sqrt{\alpha} A_{\theta}\right)-\frac{e^{2 P} A_{t}}{t \sqrt{\alpha}}\right) \\
& \quad+\left(\alpha A_{\theta \theta}+\left(-\frac{1}{t}+\frac{\alpha_{t}}{2 \alpha}\right) A_{t}+\frac{1}{2} \alpha_{\theta} A_{\theta}-2\left(A_{t} P_{t}-\alpha A_{\theta} P_{\theta}\right)\right) \frac{e^{2 P}}{\sqrt{\alpha}} A_{t} \\
& \quad+2 P_{t} e^{2 P} \sqrt{\alpha} A_{\theta}^{2}+\frac{\alpha_{t}}{2 \sqrt{\alpha}} e^{2 P} A_{\theta}^{2}+2 A_{\theta t} A_{\theta} e^{2 P} \sqrt{\alpha} \\
= & \int_{[0,1]}-\frac{1}{t} \frac{e^{2 P} A_{t}^{2}}{\sqrt{\alpha}}+A_{t} \partial_{\theta}\left(e^{2 P} \sqrt{\alpha} A_{\theta}\right)+\frac{e^{2 P}}{\sqrt{\alpha}} A_{t} \alpha A_{\theta \theta}-\frac{1}{t} \frac{e^{2 P} A_{t}^{2}}{\sqrt{\alpha}}+\frac{\alpha_{t}}{2 \alpha} \frac{e^{2 P} A_{t}^{2}}{\sqrt{\alpha}}+\frac{1}{2} \alpha_{\theta} A_{\theta} \frac{e^{2 P} A_{t}}{\sqrt{\alpha}} \\
& \quad-2 A_{t}^{2} P_{t} \frac{e^{2 P}}{\sqrt{\alpha}}+2 \alpha P_{\theta} A_{\theta} \frac{e^{2 P} A_{t}}{\sqrt{\alpha}}+2 P_{t} e^{2 P} \sqrt{\alpha} A_{\theta}^{2}+\frac{\alpha_{t}}{2 \sqrt{\alpha}} e^{2 P} A_{\theta}^{2}+2 A_{\theta t} A_{\theta} e^{2 P} \sqrt{\alpha}, \\
= & \int_{[0,1]}-2 \frac{e^{2 P} A_{t}^{2}}{\sqrt{\alpha}}+2 \partial_{\theta}\left(A_{t} e^{2 P} \sqrt{\alpha} A_{\theta}\right)+\left(\frac{\alpha_{t}}{2 \alpha}-2 P_{t}\right) \frac{e^{2 P} A_{t}^{2}}{\sqrt{\alpha}}+\left(\frac{\alpha_{t}}{2 \alpha}+2 P_{t}\right) e^{2 P} \sqrt{\alpha} A_{\theta}^{2} .
\end{aligned}
$$

Since the second term vanishes due to the periodicity, we obtain

$$
\frac{d E_{A}}{d t}=\int_{[0,1]}-2 \frac{e^{2 P} A_{t}^{2}}{\sqrt{\alpha}}+\left(\frac{\alpha_{t}}{2 \alpha}-2 P_{t}\right) \frac{e^{2 P} A_{t}^{2}}{\sqrt{\alpha}}+\left(\frac{\alpha_{t}}{2 \alpha}+2 P_{t}\right) e^{2 P} \sqrt{\alpha} A_{\theta}^{2} .
$$

Note that by assumption, the spacetime is not polarized and thus $E_{A}$ cannot identically vanish on any Cauchy surface, in particular, on any surface of constant $t$. Now, if there exists $t^{\prime} \in\left(t_{0}, t_{i}\right]$ such that, for all $(t, \theta) \in\left(t_{0}, t^{\prime}\right] \times[0,1]$, both $\left(\alpha_{t} / 2 \alpha\right)+P_{t}$ and $\left(\alpha_{t} / 2 \alpha\right)-P_{t}$ tend to 0 , it follows that $E_{A}$ is increasing in the past direction, which contradicts the fact that $E_{A} \rightarrow 0$ as $t \rightarrow t_{0}$. We are led to the following:

Lemma 8.15. There exists a constant $C>0$ and a sequence of points $\left(t_{n}, \theta_{n}\right)$ in $\left(t_{0}, t_{i}\right] \times[0,1]$, with $t_{n} \rightarrow t_{0}$, as $n \rightarrow+\infty$ such that $\frac{\left|P_{t}\right|}{\sqrt{\alpha}}\left(t_{n}, \theta_{n}\right) \geq C$.

Proof. As explained above, we have a sequence of points $\left(t_{n}, \theta_{n}\right)$ such that $\left|P_{t}\right|+\alpha_{t} /(2 \alpha) \geq 0$; otherwise $E_{A}$ is increasing for $t$ close to $t_{0}$. From Corollary 3 of Section $8 \mathrm{~J}$ and (149), there exists a constant $M>0$ such that, for all $(t, \theta) \in\left(t_{0}, t_{i}\right] \times[0,1]$,

$$
\frac{\alpha_{t}}{\alpha}(t, \theta) \leq-M \sqrt{\alpha}(t, \theta)
$$

from which we obtain

$$
\left(\left|P_{t}\right|-\frac{M}{2} \sqrt{\alpha}\right)\left(t_{n}, \theta_{n}\right) \geq 0
$$

which proves the lemma.

The set of points we have just obtained will be used as initial data for some null cone estimates, where the aim will be to estimate from below the energy density $h$. However, we will need to treat some of the 
nonlinear terms as error terms, and for this, it will be necessary to first control $h$ from above, which is the subject of the next section.

80. Pointwise null cone energy estimates: control from above. We introduce the energy density:

$$
h^{\times}=2 \sqrt{\alpha} P_{t} P_{\theta}+2 e^{2 P} \sqrt{\alpha} A_{t} A_{\theta} .
$$

Let us compute the sum and the difference of $h$ and $h^{\times}$:

$$
\begin{aligned}
& h+h^{\times}=\left(P_{t}+\sqrt{\alpha} P_{\theta}\right)^{2}+e^{2 P}\left(A_{t}+\sqrt{\alpha} A_{\theta}\right)^{2}, \\
& h-h^{\times}=\left(P_{t}-\sqrt{\alpha} P_{\theta}\right)^{2}+e^{2 P}\left(A_{t}-\sqrt{\alpha} A_{\theta}\right)^{2} .
\end{aligned}
$$

Define

$$
\begin{aligned}
D_{u} & =\partial_{t}-\sqrt{\alpha} \partial_{\theta}, \\
D_{v} & =\partial_{t}+\sqrt{\alpha} \partial_{\theta}, \\
P_{u} & =D_{u} P, \quad P_{v}=D_{v} P, \\
A_{u} & =D_{u} A, \quad A_{v}=D_{v} A .
\end{aligned}
$$

With this notation, we have

$$
\begin{aligned}
& h+h^{\times}=P_{v}^{2}+e^{2 P} A_{v}^{2}, \\
& h-h^{\times}=P_{u}^{2}+e^{2 P} A_{u}^{2} .
\end{aligned}
$$

We may also rewrite the wave equations (113) and (109) for $P$ and $A$ as ${ }^{23}$

$$
\begin{aligned}
& D_{u} D_{v} P=\frac{\alpha_{t}}{2 \alpha} P_{v}-\frac{1}{2 t}\left(P_{u}+P_{v}\right)+e^{2 P} A_{u} A_{v}-\frac{1}{2 t^{4}} \alpha e^{2 v} K^{2}, \\
& D_{v} D_{u} P=\frac{\alpha_{t}}{2 \alpha} P_{u}-\frac{1}{2 t}\left(P_{u}+P_{v}\right)+e^{2 P} A_{u} A_{v}-\frac{1}{2 t^{4}} \alpha e^{2 v} K^{2}, \\
& D_{u} D_{v} A=\frac{\alpha_{t}}{2 \alpha} A_{v}-\frac{1}{2 t}\left(A_{u}+A_{v}\right)-A_{u} P_{v}-A_{v} P_{u}, \\
& D_{v} D_{u} A=\frac{\alpha_{t}}{2 \alpha} A_{u}-\frac{1}{2 t}\left(A_{u}+A_{v}\right)-A_{u} P_{v}-A_{v} P_{u} .
\end{aligned}
$$

We have

$$
\begin{aligned}
& D_{u}\left(h+h^{\times}\right)=\left(-\frac{1}{t}+\frac{\alpha_{t}}{\alpha}\right)\left(P_{v}^{2}+e^{2 P} A_{v}^{2}\right)-\frac{1}{t}\left(P_{u} P_{v}+e^{2 P} A_{v} A_{u}\right)-\frac{P_{v}}{t^{4}} \alpha e^{2 v} K^{2}, \\
& D_{v}\left(h-h^{\times}\right)=\left(-\frac{1}{t}+\frac{\alpha_{t}}{\alpha}\right)\left(P_{u}^{2}+e^{2 P} A_{u}^{2}\right)-\frac{1}{t}\left(P_{u} P_{v}+e^{2 P} A_{v} A_{u}\right)-\frac{P_{u}}{t^{4}} \alpha e^{2 v} K^{2},
\end{aligned}
$$

or, equivalently,

$$
\begin{aligned}
& D_{u}\left(h+h^{\times}\right)=\left(-\frac{1}{t}+\frac{\alpha_{t}}{\alpha}\right)\left(h+h^{\times}\right)-\frac{1}{t}\left(P_{u} P_{v}+e^{2 P} A_{v} A_{u}\right)-\frac{P_{v}}{t^{4}} \alpha e^{2 v} K^{2}, \\
& D_{v}\left(h-h^{\times}\right)=\left(-\frac{1}{t}+\frac{\alpha_{t}}{\alpha}\right)\left(h-h^{\times}\right)-\frac{1}{t}\left(P_{u} P_{v}+e^{2 P} A_{v} A_{u}\right)-\frac{P_{u}}{t^{4}} \alpha e^{2 v} K^{2} .
\end{aligned}
$$

\footnotetext{
${ }^{23}$ Note that $D_{u} D_{v}=D_{v} D_{u}+\frac{\alpha_{t}}{\sqrt{\alpha}} \partial_{\theta}$.
} 
We will prove the next result using null cone estimates:

Lemma 8.16. For all $\epsilon>0$, there exists a constant $B>0, a t^{\prime}>t_{0}$ and $a \theta_{0} \in[0,1]$ such that, for all $t^{\prime} \geq t>t_{0}$,

$$
\sup _{\theta \in[0,1]} h(t, \cdot) \leq B \alpha^{1+\epsilon}\left(t, \theta_{0}\right) .
$$

Proof. Let $t \in\left(t_{0}, t_{i}\right]$ and let $\Theta(s, \theta, t)$ denote a solution of the characteristic equation with initial conditions $\Theta(t, \theta, t)=\theta$ such that $(s, \Theta(s, \theta, t))$ corresponds to a constant $v$ line in null coordinates, as introduced in Section $8 \mathrm{~K}$.

We have

$$
\frac{\partial}{\partial s}\left(\left(h+h^{\times}\right)(s, \Theta(s, \theta, t))\right)=\frac{\partial\left(h+h^{\times}\right)}{\partial t}-\sqrt{\alpha} \frac{\partial\left(h+h^{\times}\right)}{\partial \theta}=D_{u}\left(h+h^{\times}\right)(s, \Theta(s, \theta, t))
$$

and therefore (207) can be rewritten as follows, for any $t^{\prime}>t_{0}$ :

$$
\begin{aligned}
\frac{\partial}{\partial s}\left(\left(h+h^{\times}\right)(s, \Theta(s, \theta, t)) \exp \int_{s}^{t^{\prime}}\right. & \left.\left(-\frac{1}{s^{\prime}}+\frac{\alpha_{t}}{\alpha}\right)\left(s^{\prime}, \Theta\left(s^{\prime}, \theta, t\right)\right) d s^{\prime}\right) \\
& =\left(\exp \int_{s}^{t^{\prime}}\left(-\frac{1}{s^{\prime}}+\frac{\alpha_{t}}{\alpha}\right)\left(s^{\prime}, \Theta\left(s^{\prime}, \theta, t\right)\right) d s^{\prime}\right) \phi(s, \Theta(s, \theta, t)),
\end{aligned}
$$

where

$$
\phi=-\frac{1}{s}\left(P_{u} P_{v}+e^{2 P} A_{v} A_{u}\right)-\frac{P_{v}}{s^{4}} \alpha e^{2 v} K^{2} .
$$

Let $t^{\prime} \geq t>t_{0}$ and integrate the last line between $t^{\prime}$ and $t$ to obtain

$$
\begin{aligned}
\left(h+h^{\times}\right)\left(t^{\prime}, \Theta\left(t^{\prime}, \theta, t\right)\right)- & \left(h+h^{\times}\right)(t, \theta) \exp \int_{t}^{t^{\prime}}\left(-\frac{1}{s^{\prime}}+\frac{\alpha_{t}}{\alpha}\right)\left(s^{\prime}, \Theta\left(s^{\prime}, \theta, t\right)\right) d s^{\prime} \\
& =\int_{t}^{t^{\prime}}\left[\left(\exp \int_{s}^{t^{\prime}}\left(-\frac{1}{s^{\prime}}+\frac{\alpha_{t}}{\alpha}\right)\left(s^{\prime}, \Theta\left(s^{\prime}, \theta, t\right)\right) d s^{\prime}\right) \phi(s, \Theta(s, \theta, t))\right] d s .
\end{aligned}
$$

Let $\epsilon>0$ and fix a $\theta_{0}$ in $[0,1]$. Assume $t^{\prime}$ is such that Lemma 8.10 holds in the following sense: for all $(t, \theta) \in\left(t_{0}, t^{\prime}\right] \times[0,1]$,

$$
(1+\epsilon) \frac{\alpha_{t}}{\alpha}\left(t, \theta_{0}\right) \leq \frac{\alpha_{t}}{\alpha}(t, \theta) \leq(1-\epsilon) \frac{\alpha_{t}}{\alpha}\left(t, \theta_{0}\right),
$$

which implies the estimates

$$
\frac{t}{t^{\prime}}\left(\frac{\alpha\left(t^{\prime}, \theta_{0}\right)}{\alpha\left(t, \theta_{0}\right)}\right)^{1+\epsilon} \leq \exp \int_{t}^{t^{\prime}}\left(-\frac{1}{s^{\prime}}+\frac{\alpha_{t}}{\alpha}\right)\left(s^{\prime}, \Theta\left(s^{\prime}, \theta, t\right)\right) d s^{\prime}
$$

and

$$
\exp \int_{t}^{t^{\prime}}\left(-\frac{1}{s^{\prime}}+\frac{\alpha_{t}}{\alpha}\right)\left(s^{\prime}, \Theta\left(s^{\prime}, \theta, t\right)\right) d s^{\prime} \leq \frac{t}{t^{\prime}}\left(\frac{\alpha\left(t^{\prime}, \theta_{0}\right)}{\alpha\left(t, \theta_{0}\right)}\right)^{1-\epsilon} .
$$

Define $F(s, \theta, t)$ by

$$
F(s, \theta, t)=\left(h+h^{\times}\right)(s, \Theta(s, \theta, t)) \frac{s}{t^{\prime}}\left(\frac{\alpha\left(t^{\prime}, \theta_{0}\right)}{\alpha\left(s, \theta_{0}\right)}\right)^{1+\epsilon} .
$$


Note that it follows from the definition of $F(s, \theta, t)$ that, for all $t, t^{\prime} \in\left(t_{0}, t_{i}\right]$,

$$
\sup _{\theta \in[0,1]} F(s, \theta, t)=\sup _{\theta \in[0,1]} F\left(s, \theta, t^{\prime}\right)
$$

Thus, we may define $Z(s)$ by

$$
Z(s)=\sup _{\theta \in[0,1]} F(s, \theta, t)
$$

From (212), (214) and (215), we have

$$
F(t, \theta, t) \leq F\left(t^{\prime}, \theta, t\right)+\int_{t}^{t^{\prime}} \frac{s}{t^{\prime}}\left(\frac{\alpha\left(t^{\prime}, \theta_{0}\right)}{\alpha\left(s, \theta_{0}\right)}\right)^{1-\epsilon}|\phi(s, \Theta(s, \theta, t))| d s .
$$

We will now estimate the second term on the right-hand side of the last inequality. First note that

$$
\begin{aligned}
|\phi(s, \Theta(s, \theta, t))| & =\left|-\frac{1}{s}\left(P_{u} P_{v}+e^{2 P} A_{v} A_{u}\right)-\frac{P_{v}}{s^{4}} \alpha e^{2 v} K^{2}\right| \\
& \leq \frac{h}{s}+\sqrt{h+h^{\times}} \frac{e^{2 \beta} K^{2}}{s^{4}} .
\end{aligned}
$$

Thus

$$
\left(\frac{\alpha\left(t^{\prime}, \theta_{0}\right)}{\alpha\left(s, \theta_{0}\right)}\right)^{1-\epsilon}|\phi(s, \Theta(s, \theta))| \leq\left(\frac{\alpha\left(t^{\prime}, \theta_{0}\right)}{\alpha\left(s, \theta_{0}\right)}\right)^{1-\epsilon} \frac{1}{2} h+\left(\frac{\alpha\left(t^{\prime}, \theta_{0}\right)}{\alpha\left(s, \theta_{0}\right)}\right)^{1-\epsilon} \sqrt{h+h^{\times}} \frac{e^{2 \beta} K^{2}}{s^{4}} .
$$

The second term on the right-hand side of this last line may then be rewritten in terms of $F(s, \theta, t)$ :

$$
\left(\frac{\alpha\left(t^{\prime}, \theta_{0}\right)}{\alpha\left(s, \theta_{0}\right)}\right)^{1-\epsilon} \sqrt{h+h^{\times}} \frac{e^{2 \beta} K^{2}}{s^{4}}=\left(\frac{\alpha\left(t^{\prime}, \theta_{0}\right)}{\alpha\left(s, \theta_{0}\right)}\right)^{1 / 2-3 \epsilon / 2} \frac{e^{2 \beta} K^{2}}{s^{4}}\left(\frac{t^{\prime}}{s}\right)^{1 / 2} \sqrt{F(s, \theta, t)} .
$$

Moreover, from Lemmas 8.5 and 8.14 and Corollary 3, for $\epsilon$ small enough we have

$$
\int_{t}^{t^{\prime}}\left(\frac{\alpha\left(t^{\prime}, \theta_{0}\right)}{\alpha\left(s, \theta_{0}\right)}\right)^{1 / 2-3 \epsilon / 2} \frac{e^{2 \beta} K^{2}}{s^{4}}\left(\frac{t^{\prime}}{s}\right)^{1 / 2} d s \leq \int_{t}^{t^{\prime}} C \alpha^{(3 \epsilon) / 2}\left(s, \theta_{0}\right) d s \leq M,
$$

for some constant $M>0$. We now use estimates of the type found in [Smulevici 2008]. Let $t_{m}$ be such that $Z\left(t_{m}\right)$ is a maximum of $Z$ on $\left[t, t^{\prime}\right]$. Note the trivial fact that $\sup _{\left[t, t^{\prime}\right]} Z=Z\left(t_{m}\right)=\sup _{\left[t_{m}, t^{\prime}\right]} Z$.

It follows from (217), (220) and (222) that

$$
F\left(t_{m}, \theta, t_{m}\right) \leq F\left(t^{\prime}, \theta, t_{m}\right)+\sqrt{Z\left(t_{m}\right)} M+\int_{t_{m}}^{t^{\prime}} \frac{s}{t^{\prime}}\left(\frac{\alpha\left(t^{\prime}, \theta_{0}\right)}{\alpha\left(s, \theta_{0}\right)}\right)^{1-\epsilon} \frac{h\left(s, \Theta\left(s, \theta, t_{m}\right)\right)}{s} d s,
$$

for some constant $M>0$. Note that $F\left(t^{\prime}, \theta, t_{m}\right)$ is uniformly bounded since, by definition,

$$
F\left(t^{\prime}, \theta, t_{m}\right)=\left(h+h^{\times}\right)\left(t^{\prime}, \Theta\left(t^{\prime}, \theta, t_{m}\right)\right) \leq \sup _{\theta \in[0,1]}\left(h+h^{\times}\right)\left(t^{\prime}, \cdot\right) \leq C
$$

for some constant $C>0$. Thus we have, from (223),

$$
F\left(t_{m}, \theta, t_{m}\right) \leq C+\sqrt{Z\left(t_{m}\right)} M+\int_{t_{m}}^{t^{\prime}} \frac{s}{t^{\prime}}\left(\frac{\alpha\left(t^{\prime}, \theta_{0}\right)}{\alpha\left(s, \theta_{0}\right)}\right)^{1-\epsilon} \frac{h\left(s, \Theta\left(s, \theta, t_{m}\right)\right)}{s} d s
$$


and taking the supremum over $\theta$, we obtain

$$
Z\left(t_{m}\right) \leq C+\sqrt{Z\left(t_{m}\right)} M+D \int_{t_{m}}^{t^{\prime}}\left(\frac{\alpha\left(t^{\prime}, \theta_{0}\right)}{\alpha\left(s, \theta_{0}\right)}\right)^{1-\epsilon} \sup _{\theta \in[0,1]}(h(s, \cdot)) d s .
$$

We interpret the last line as an inequality for a second-order polynomial equation in $\sqrt{Z\left(t_{m}\right)}$. Thus $\sqrt{Z\left(t_{m}\right)}$ must lie between the roots of this polynomial, and we obtain easily that

$$
Z\left(t_{m}\right) \leq C^{\prime}+C^{\prime} \int_{t_{m}}^{t^{\prime}}\left(\frac{\alpha\left(t^{\prime}, \theta_{0}\right)}{\alpha\left(s, \theta_{0}\right)}\right)^{1-\epsilon} \sup _{\theta \in[0,1]}(h(s, \cdot)) d s
$$

for some constant $C^{\prime}>0$. Since $Z(t) \leq Z\left(t_{m}\right)$ and since $t \leq t_{m}$, we have

$$
Z(t) \leq C^{\prime}+C^{\prime} \int_{t}^{t^{\prime}}\left(\frac{\alpha\left(t^{\prime}, \theta_{0}\right)}{\alpha\left(s, \theta_{0}\right)}\right)^{1-\epsilon} \sup _{\theta \in[0,1]}(h(s, \cdot)) d s
$$

Thus, we have established that

$$
\left(\sup _{\theta \in[0,1]}\left(h+h^{\times}\right)(t, \cdot)\right) \frac{t}{t^{\prime}}\left(\frac{\alpha\left(t^{\prime}, \theta_{0}\right)}{\alpha\left(t, \theta_{0}\right)}\right)^{1+\epsilon} \leq B+C \int_{t}^{t^{\prime}}\left(\frac{\alpha\left(t^{\prime}, \theta_{0}\right)}{\alpha\left(s, \theta_{0}\right)}\right)^{1-\epsilon} \sup _{\theta \in[0,1]}(h(s, \cdot)) d s,
$$

for some constants $B, C>0$. A similar estimate may be obtained using $h-h^{\times}$and (208). Adding the estimate for $h-h^{\times}$to (229), we obtain easily that

$$
\left(\sup _{\theta \in[0,1]} h(t, \cdot)\right) \frac{t}{t^{\prime}}\left(\frac{\alpha\left(t^{\prime}, \theta_{0}\right)}{\alpha\left(t, \theta_{0}\right)}\right)^{1+\epsilon} \leq B+C \int_{t}^{t^{\prime}}\left(\frac{\alpha\left(t^{\prime}, \theta_{0}\right)}{\alpha\left(t, \theta_{0}\right)}\right)^{1-\epsilon} \sup _{\theta \in[0,1]}(h(s, \cdot)) d s .
$$

Applying Gronwall's lemma, together with Lemma 8.14, completes the proof.

8P. Pointwise null cone energy estimates: control from below. With the control from above for $h$ that we have just obtained, we may now prove an estimate from below for $h$ if we have appropriate initial data:

Lemma 8.17. Suppose that there exists a constant $B>0$ and a sequence of points $\left(t_{n}, \theta_{n}\right)$ with $t_{n} \rightarrow t_{0}$ as $n \rightarrow+\infty$, such that

$$
\frac{\left|P_{v}\right|}{\sqrt{\alpha}}\left(t_{n}, \theta_{n}\right)>B \quad \text { for all } n .
$$

For all $\epsilon>0$, there exists $C>0, t^{\prime}>t_{0}, \theta^{\prime} \in[0,1]$ and an interval $\left[\theta^{\prime}-\delta, \theta^{\prime}+\delta\right]$ with $\delta>0$ such that, for all $(t, \theta) \in\left(t_{0}, t^{\prime}\right] \times\left[\theta^{\prime}-\delta, \theta^{\prime}+\delta\right]$,

$$
h\left(t, \Theta\left(t, \theta, t^{\prime}\right)\right) \geq C \alpha^{1-\epsilon}\left(t, \Theta\left(t, \theta, t^{\prime}\right)\right),
$$

where $\left(s, \Theta\left(s, \theta, t^{\prime}\right)\right)$ denote the parametrizations of the null lines parallel to the constant $v$ lines starting at $\left(t^{\prime}, \theta\right)$ which were introduced in Section $8 K$.

Proof. Let $\epsilon>0$ and $n_{0} \in \mathbb{N}$ be such that Lemma 8.16 holds and Lemma 8.10 holds as in (213), with $t^{\prime}$ replaced by $t_{n_{0}}$ in both lemmas. Let $n \geq n_{0}$. We will integrate (207) in a way similar to the proof of the last lemma. Let us denote by $\Theta_{n}(t, \theta)$ the null lines parallel to the constant $v$ lines starting at $\left(t_{n}, \theta\right)$, i.e., $\Theta_{n}(t, \theta)=\Theta\left(t, \theta, t_{n}\right)$. Equation (207) can then be integrated as 


$$
\begin{aligned}
\left(h+h^{\times}\right)\left(t_{n}, \theta\right)-\left(h+h^{\times}\right) & \left(t, \Theta_{n}(t, \theta)\right) \exp \int_{t}^{t_{n}}\left(-\frac{1}{s^{\prime}}+\frac{\alpha_{t}}{\alpha}\right)\left(s^{\prime}, \Theta_{n}\left(s^{\prime}, \theta\right)\right) d s^{\prime} \\
= & \int_{t}^{t_{n}}\left(\left(\exp \int_{s}^{t_{n}}\left(-\frac{1}{s^{\prime}}+\frac{\alpha_{t}}{\alpha}\right)\left(s^{\prime}, \Theta_{n}\left(s^{\prime}, \theta\right)\right) d s^{\prime}\right) \phi\left(s, \Theta_{n}(s, \theta)\right)\right) d s,
\end{aligned}
$$

where

$$
\phi=-\frac{1}{s}\left(P_{u} P_{v}+e^{2 P} A_{v} A_{u}\right)-\frac{P_{v}}{s^{4}} \alpha e^{2 v} K^{2} .
$$

Fix $\theta_{0} \in[0,1]$. Since Lemma 8.10 holds in the sense of (213) for $t \in\left(t_{0}, t_{n}\right]$, we have again the estimates

$$
\begin{aligned}
& \frac{t}{t_{n}}\left(\frac{\alpha\left(t_{n}, \theta_{0}\right)}{\alpha\left(t, \theta_{0}\right)}\right)^{1+\epsilon} \leq \exp \int_{t}^{t_{n}}\left(\frac{-1}{s}+\frac{\alpha_{t}}{\alpha}\right)\left(s, \Theta_{n}(s, \theta)\right) d s, \\
& \exp \int_{t}^{t_{n}}\left(\frac{-1}{s}+\frac{\alpha_{t}}{\alpha}\right)\left(s, \Theta_{n}(s, \theta)\right) d s \leq \frac{t}{t_{n}}\left(\frac{\alpha\left(t_{n}, \theta_{0}\right)}{\alpha\left(t, \theta_{0}\right)}\right)^{1-\epsilon} .
\end{aligned}
$$

Using this, we may estimate the last term on the right-hand side of (231):

$$
\begin{aligned}
\int_{t}^{t_{n}}\left(\left(\exp \int_{s}^{t_{n}}\left(-\frac{1}{s^{\prime}}+\frac{\alpha_{t}}{\alpha}\right)\left(s^{\prime}, \Theta_{n}\left(s^{\prime}, \theta\right)\right) d s^{\prime}\right) \phi\left(s, \Theta_{n}(s, \theta)\right)\right) d s \\
\leq \int_{t}^{t_{n}} \frac{t}{t_{n}}\left(\frac{\alpha\left(t_{n}, \theta_{0}\right)}{\alpha\left(t, \theta_{0}\right)}\right)^{1-\epsilon}|\phi|\left(s, \Theta_{n}(s, \theta)\right) d s \\
\leq \int_{t}^{t_{n}} \frac{t}{t_{n}}\left(\frac{\alpha\left(t_{n}, \theta_{0}\right)}{\alpha\left(t, \theta_{0}\right)}\right)^{1-\epsilon}\left(\frac{h}{s}+\sqrt{h+h^{\times}} \frac{e^{2 \beta} K^{2}}{s^{4}}\right) d s
\end{aligned}
$$

We now use Lemma 8.14 and the estimates $h+h^{\times} \leq 2 h, h \leq C \alpha^{1+\epsilon}, \sqrt{h} \leq \sqrt{C} \sqrt{\alpha^{1+\epsilon}}$, and $e^{2 \beta} \leq C \sqrt{\alpha}$, for some constant $C>0$ independent of $n$, to obtain

$$
\begin{aligned}
\int_{t}^{t_{n}}\left(\left(\exp \int_{s}^{t_{n}}\left(-\frac{1}{s^{\prime}}+\frac{\alpha_{t}}{\alpha}\right)\left(s^{\prime}, \Theta_{n}\left(s^{\prime}, \theta\right)\right) d s^{\prime}\right) \phi\left(s, \Theta_{n}(s, \theta)\right)\right) d s & \leq \alpha\left(t_{n}, \theta_{0}\right)^{1-\epsilon} \int_{t}^{t_{n}} C \alpha^{2 \epsilon} \\
& \leq C_{n}^{\prime} \alpha\left(t_{n}, \theta_{0}\right),
\end{aligned}
$$

where $C_{n}^{\prime} \rightarrow 0$ as $n \rightarrow \infty$.

We obtain from (231) that

$$
\begin{aligned}
& \left(h+h^{\times}\right)\left(t, \Theta_{n}(t, \theta)\right) \geq\left(\left(h+h^{\times}\right)\left(t_{n}, \theta\right)-C_{n}^{\prime}(t) \alpha\left(t_{n}, \theta_{0}\right)\right) \exp \int_{t}^{t_{n}}\left(\frac{1}{s^{\prime}}-\frac{\alpha_{t}}{\alpha}\right)\left(s^{\prime}, \Theta_{n}\left(s^{\prime}, \theta\right)\right) d s^{\prime}, \\
& \left(h+h^{\times}\right)\left(t, \Theta_{n}(t, \theta)\right) \geq\left(\left(h+h^{\times}\right)\left(t_{n}, \theta\right)-C_{n}^{\prime}(t) \alpha\left(t_{n}, \theta_{0}\right)\right) \frac{t_{n}}{t}\left(\frac{\alpha\left(t, \theta_{0}\right)}{\alpha\left(t_{n}, \theta_{0}\right)}\right)^{1-\epsilon} .
\end{aligned}
$$

By assumption, for all $n \in \mathbb{N}$, we have

$$
\frac{\left|P_{v}\right|}{\sqrt{\alpha}}\left(t_{n}, \theta_{n}\right)>B
$$

and thus, from (199), $\left(h+h^{\times}\right)\left(t_{n}, \theta_{n}\right) / \alpha\left(t_{n}, \theta_{n}\right) \geq A$, for some $A>0$. By application of Corollary 2, we obtain $\left(h+h^{\times}\right)\left(t_{n}, \theta_{n}\right) / \alpha\left(t_{n}, \theta_{0}\right) \geq A^{\prime}$, for some constant $A^{\prime}>0$. Thus, for all $n \in \mathbb{N}$, there exists an 
interval around $\theta_{n}$, say $\left[\theta_{n}-\delta_{n}, \theta_{n}+\delta_{n}\right]$ with $\delta_{n}>0$, such that, for all $\theta \in\left[\theta_{n}-\delta_{n}, \theta_{n}+\delta_{n}\right]$,

$$
\frac{\left(h+h^{\times}\right)\left(t_{n}, \theta\right)}{\alpha\left(t_{n}, \theta_{0}\right)} \geq \frac{A^{\prime}}{2} .
$$

Let $n_{1}$ be such that for all $n \geq n_{1}$ and all $t \in\left(t_{0}, t_{n}\right], C_{n}^{\prime} \leq A^{\prime} / 4$. Let $n_{2}=\max \left(n_{0}, n_{1}\right)$. Then we have, from (237) and (238), for all $(t, \theta) \in\left(t_{0}, t_{n_{2}}\right] \times\left[\theta_{n_{2}}-\delta_{n_{2}}, \theta_{n_{2}}+\delta_{n_{2}}\right]$,

$$
\left(h+h^{\times}\right)\left(t, \Theta_{n_{2}}(t, \theta)\right) \geq \frac{A^{\prime}}{4} \alpha\left(t_{n_{2}}, \theta_{0}\right) \frac{t_{n_{2}}}{t}\left(\frac{\alpha\left(t, \theta_{0}\right)}{\alpha\left(t_{n_{2}}, \theta_{0}\right)}\right)^{1-\epsilon} .
$$

Moreover, we have $\alpha\left(t, \Theta_{n_{2}}(t, \theta)\right) \leq M \alpha\left(t, \theta_{0}\right)$ for some constant $M>0$; thus we obtain

$$
\left(h+h^{\times}\right)\left(t, \Theta_{n_{2}}(t, \theta)\right) \geq \frac{A^{\prime}}{4 M^{1-\epsilon}} \alpha\left(t_{n_{2}}, \theta_{0}\right) \frac{t_{n_{2}}}{t_{0}}\left(\frac{\alpha\left(t, \Theta_{n_{2}}(t, \theta)\right)}{\alpha\left(t_{n_{2}}, \theta_{0}\right)}\right)^{1-\epsilon},
$$

which proves the lemma.

Remark 8P.1. With the notation of Lemma 8.17, it is possible to choose $t^{\prime}$ so that $t^{\prime} \in\left(t_{0}, \bar{t}\right]$, where $\bar{t}$ is such that Lemma 8.12 holds. To see this, just replace in the above proof $n_{0}$ by $n_{0}^{\prime} \geq n_{0}$ such that $t_{n_{0}^{\prime}} \in\left(t_{0}, \bar{t}\right]$.

8Q. The contradiction. From Lemma 8.15, there exists a sequence of points $\left(t_{n}, \theta_{n}\right)$ and a constant $A>0$ such that $\frac{\left|P_{t}\right|}{\sqrt{\alpha}}\left(t_{n}, \theta_{n}\right) \geq A$. Thus, without of generality, we may assume that there exists a sequence of points $\left(t_{n}^{\prime}, \theta_{n}^{\prime}\right)$ and a constant $A>0$ such $\frac{\left|P_{v}\right|}{\sqrt{\alpha}}\left(t_{n}^{\prime}, \theta_{n}^{\prime}\right) \geq \frac{A}{2}$, exchanging the role of $u$ and $v$ if necessary. Therefore, Lemma 8.17 applies, and for all $\epsilon>0$, there exists a $C>0$, a $t^{\prime}>t_{0}$, a $\theta^{\prime} \in[0,1]$ and an interval $\left[\theta^{\prime}-\delta, \theta^{\prime}+\delta\right]$ with $\delta>0$ such that, for all $(t, \theta) \in\left(t_{0}, t^{\prime}\right] \times\left[\theta^{\prime}-\delta, \theta^{\prime}+\delta\right]$,

$$
h\left(t, \Theta\left(t, \theta, t^{\prime}\right)\right) \geq C \alpha^{1-\epsilon}\left(t, \Theta\left(t, \theta, t^{\prime}\right)\right),
$$

where $\left(s, \Theta\left(s, \theta, t^{\prime}\right)\right)$ denote the parametrizations of the null lines parallel to the constant $v$ lines, starting at $\left(t^{\prime}, \theta\right)$. Moreover, let us choose $t^{\prime}$ so that $t^{\prime} \in\left(t_{0}, \bar{t}\right]$, where $\bar{t}$ is such that Lemma 8.12 holds, as in Remark 8P.1.

Consider the integral in $\theta$ of $h\left(t, \Theta\left(t, \theta, t^{\prime}\right)\right)$ and fix a $\theta_{0} \in[0,1]$. We have

$$
\int_{[0,1]} h\left(t, \Theta\left(t, \theta, t^{\prime}\right)\right) d \theta \geq 2 \delta C \alpha^{1-\epsilon}\left(t, \theta_{0}\right),
$$

using Corollary 2. On the other hand, we have, by the change of variable $\theta^{\prime}=\Theta\left(t, \theta, t^{\prime}\right)$,

$$
\int_{[0,1]} h\left(t, \Theta\left(t, \theta, t^{\prime}\right)\right) d \theta=\int_{[0,1]} h\left(t, \theta^{\prime}\right) \Theta_{\theta}^{-1} d \theta^{\prime} .
$$

From (169), we therefore have

$$
\int_{[0,1]} h\left(t, \Theta\left(t, \theta, t^{\prime}\right)\right) d \theta=\int_{[0,1]} h\left(t, \theta^{\prime}\right)\left(\exp \int_{t^{\prime}}^{t} \frac{1}{2}\left(\frac{\alpha_{\theta}}{\sqrt{\alpha}}\right) d s\right) d \theta^{\prime},
$$

where the integral in the exponential is taken along the characteristics. 
Since Lemma 8.12 holds, we have

$$
\exp \int_{t^{\prime}}^{t} \frac{1}{2}\left(\frac{\alpha_{\theta}}{\sqrt{\alpha}}\right) d s \leq M \alpha^{\epsilon}
$$

Thus, we obtain

$$
\int_{[0,1]} h\left(t, \Theta\left(t, \theta, t^{\prime}\right)\right) d \theta \leq \int_{[0,1]} h M \alpha^{\epsilon}\left(t, \theta^{\prime}\right) d \theta^{\prime} .
$$

Using again Corollary 2 and the boundedness of $E_{h}=\int_{[0,1]}(h / \sqrt{\alpha}) d \theta$, we see that the right-hand of the last inequality is bounded by $M^{\prime} \alpha^{1 / 2+\epsilon}\left(t, \theta_{0}\right)$ for some constant $M^{\prime}$. Choosing $\epsilon$ small enough, this contradicts (242) since $\alpha \rightarrow \infty$ as $t \rightarrow t_{0}$. Thus Theorem 2 is proved. ${ }^{24}$

\section{Proof of Theorem 3}

We will prove Theorem 3 in this section. For this, we will adapt the proof found by Isenberg and Weaver [2003] to the case of $k=-1$ surface-symmetric spacetimes. To exploit their methods, we have rewritten the metric in a form similar to the $T^{2}$ case (see (7) in Section 2B). In particular, the coordinate $t$ used in (7) denotes the square of the usual areal time used for these spacetimes, as found for instance in [Tchapnda 2004].

To start, we recall the Einstein-Vlasov system for spacetimes with a hyperbolic surface of symmetry.

9A. Vlasov matter in $\boldsymbol{k}=-1$ surface-symmetric spacetimes. Let $(\mathcal{M}, g, f)$ be a past development of $k=-1$ surface-symmetric initial data with Vlasov matter as described in Section 2D and assume that $(t, \theta, x, y)$ is a system of areal coordinates such that the metric in $M$ takes the form (7). Let $v_{i}$, $i=0,1,2,3$ denotes the components of the velocity vector in the canonical basis of 1-forms associated with the coordinate system $(t, \theta, x, y)$. We will parametrize the mass shell $\mathscr{P}$ by the coordinates $\left(t, \theta, x, y, v_{1}, v_{2}, v_{3}\right)$, where by an abuse of notation, we denote the lift to $\mathscr{P}$ of the coordinates on $\mathcal{M}$ by the same symbols. The Vlasov field $f$ can be seen as a function of $\left(t, \theta, x, y, v_{1}, v_{2}, v_{3}\right)$ or, using the symmetry, as a function depending only on $t, \theta, w=\left(\sqrt{t} / e^{v}\right) v_{1}$ and $L=\gamma^{a b} v_{a} v_{b}$, and we will, by an abuse of notation, use both definitions and denote it by $f$ in either case. ${ }^{25}$

With these definitions, the mass shell relation $v_{\mu} v^{\mu}=-1$ is given by

$$
v_{0}=-\sqrt{\frac{\alpha}{t} e^{2 v}+\alpha v_{1}^{2}+\frac{\alpha e^{2 v}}{t^{2}} \gamma^{a b} v_{a} v_{b}}=-\frac{\sqrt{\alpha} e^{v}}{\sqrt{t}} \sqrt{1+w^{2}+L / t}
$$

and the Vlasov equation for $f(t, \theta, w)$ reads as

$$
2 \sqrt{t} \partial_{t} f+\frac{2 \sqrt{t \alpha} w}{\sqrt{1+w^{2}+L / t}} \partial_{\theta} f-\left(\sqrt{t}\left(2 v_{t}-1 / t\right) w+\left(v_{\theta}+\frac{\alpha_{\theta}}{2 \alpha}\right) 2 \sqrt{t \alpha} \sqrt{1+w^{2}+L / t}\right) \partial_{w} f=0 .
$$

\footnotetext{
${ }^{24}$ We see that the margin of error is, up to $\epsilon, \alpha^{1 / 2}$. This margin follows from our estimates because, up to $\alpha^{\epsilon}$, we have $h \sim \alpha$ along certain characteristics. On the other hand, if we did not have this margin, i.e., if we had $h \sim \alpha^{1 / 2}$, then it would follow that for $t^{\prime}$ close enough to $t_{0},\left(\alpha_{t} / 2 \alpha\right) \pm P_{t} \leq 0$ for all $\theta \in[0,1]$, and (189) would the imply that that $E_{A}$ is increasing the past. This would contradict the fact that $E_{A} \rightarrow 0$ as $t \rightarrow t_{0}$.

${ }^{25}$ The indices on the velocities $v_{i}$ are raised or lowered using the metric (7), not using $\gamma_{a b}$. This implies that if $p^{a}$ denotes the canonical momentum associated with the coordinates system $(t . \theta . x . y)$, then $L=t^{2} \gamma_{a b} p^{a} p^{b}$.
} 
9B. The Einstein equations. The Einstein equations (1) reduce to the following system of equations: Constraint equations:

$$
\begin{aligned}
v_{t} & =\frac{1}{4 t}+\alpha e^{2 v} \Lambda-\frac{k \alpha e^{2 v}}{t}+8 \pi \sqrt{\alpha} \int_{\mathbb{R}^{3}} f\left|v_{0}\right| \gamma^{-1 / 2} d v_{1} d v_{2} d v_{3}, \\
\frac{\alpha_{t}}{\alpha} & =-4 \Lambda \alpha e^{2 v}+\frac{4 k \alpha e^{2 v}}{t}-16 \pi \alpha^{3 / 2} e^{2 v} \int_{\mathbb{R}^{3}} \frac{f\left(t^{-1}+t^{-2}\right)}{\left|v_{0}\right|} \gamma^{-1 / 2} d v_{1} d v_{2} d v_{3}, \\
v_{\theta}+\frac{\alpha_{\theta}}{2 \alpha} & =-8 \pi \sqrt{\alpha} \int_{\mathbb{R}^{3}} f v_{1} \gamma^{-1 / 2} d v_{1} d v_{2} d v_{3} .
\end{aligned}
$$

Evolution equation:

$$
v_{t t}-\alpha v_{\theta \theta}=\frac{1}{2} \alpha_{\theta \theta}-\frac{\alpha_{\theta}^{2}}{4 \alpha}+\frac{v_{\theta} \alpha_{\theta}}{2}-\frac{1}{4 t^{2}}+\frac{\alpha_{t} v_{t}}{2 \alpha}+\frac{\alpha e^{2 v} \Lambda}{t}-4 \pi \frac{\alpha^{3 / 2} e^{2 v}}{t^{3}} \int_{\mathbb{R}^{3}} \frac{f L}{\left|v_{0}\right|} \gamma^{-1 / 2} d v_{1} d v_{2} d v_{3} .
$$

Here $k$ denotes the curvature of the surface of symmetry and will therefore be -1 in the case of hyperbolic symmetry. $\gamma$ denotes the determinant of the metric $\gamma_{a b}$.

In the rest of this section, $(\mathcal{M}, g, f)$ will be a past development of $k=-1$ surface-symmetric initial data with Vlasov matter and $\Lambda \geq 0$. We will cover $(M, g)$ by areal coordinates $(t, \theta, x, y)$, where the range of the coordinates $(t, \theta)$ is $\left(t_{f}, t_{i}\right] \times[0,1]$ with $0<t_{f}<t_{i}$. The metric will be given by (7) with the functions $\alpha$ and $v$ depending only on $(t, \theta)$ and being periodic in $\theta$ with period 1 . The Einstein-Vlasov system implies that the system (249)-(252) completed with (248) holds for all $(t, \theta) \in\left(t_{f}, t_{i}\right] \times[0,1]$. Moreover, we will assume that $f$ does not vanish identically. Following what has been said in Section 6, we will prove that for all such $(\mathcal{M}, g, f)$, the hypotheses of Proposition 2 are satisfied, from which Theorem 3 follows immediately.

First, we recall some properties of the Vlasov field for such spacetimes.

9C. Conservation laws. As in Section 7C, since $f$ is conserved along geodesics, we have an immediate upper bound on $f$ :

$$
f \leq F,
$$

for some $F>0$. Since by assumption, $f$ has compact support, conservation of angular momentum along geodesics implies an upper bound on the support of $f$ in $L$; that is, we have

$$
X=\sup _{L \in \operatorname{supp}(f)} L<\infty
$$

The particle current is given by

$$
N^{\mu}=\frac{\sqrt{\alpha}}{t} \int_{\mathbb{R}^{3}} \frac{f}{\left|v_{0}\right|} v^{\mu} \gamma^{-1 / 2} d v_{1} d v_{2} d v_{3} .
$$

From the Vlasov equation it follows that $N^{\mu}$ is divergence free $\nabla_{\mu} N^{\mu}=0$ and therefore, we have the conservation law,

$$
\int_{[0,1]} N^{0} \sqrt{\alpha} e^{2 v} d \theta=\int_{[0,1]}\left(\int_{\mathbb{R}^{3}} f \gamma^{-1 / 2} d v_{1} d v_{2} d v_{3}\right) d \theta=Q \text { for all } t,
$$

for some nonnegative constant $Q$. Moreover, since by assumption, the Vlasov field does not vanish 
identically, we have

$$
Q>0
$$

9D. Lower bound on the mean value of $\left|v_{1}\right|$. The proof of Lemma 7.1 is easily adapted to the present setting to give:

Lemma 9.1. There exists $\delta>0$ such that, for all $t$,

$$
\int_{[0,1]}\left(\int_{\mathbb{R}^{3}} f \gamma^{-1 / 2}\left|v_{1}\right| d v_{1} d v_{2} d v_{3}\right) d \theta>\delta .
$$

9E. Energy estimates. We define $E(t)$ as the energy integral

$$
E(t)=\int_{[0,1]} \frac{v_{t}}{t \sqrt{\alpha}} d \theta
$$

Lemma 9.2. E admits a continuous extension to $t_{f}$. In particular $E$ is uniformly bounded on $\left(t_{f}, t_{i}\right]$.

Proof. As usual, we take the time derivative of $E$ and use the Einstein equations and the periodicity to simplify the resulting equations. It follows that

$$
\frac{d E}{d t}=-\int_{[0,1]}\left(\frac{1}{2 t^{3} \sqrt{\alpha}}-\frac{k \sqrt{\alpha} e^{2 v}}{t^{3}}+8 \pi \int_{\mathbb{R}^{3}}\left(\frac{f\left|v_{0}\right|}{t^{2}}+\frac{\alpha e^{2 v} f L}{2 t^{4}\left|v_{0}\right|}\right) \gamma^{-1 / 2} d v_{1} d v_{2} d v_{3}\right) d \theta .
$$

Since $k=-1$, we see that $E$ is increasing with decreasing $t$. Moreover from the last equation, the definition of $E$, and (249), it follows that

$$
\frac{d E}{d t} \geq-\frac{4 E}{t}
$$

and by integration of the last line, we obtain an upper bound for $E$ on $\left(t_{f}, t_{i}\right]$.

\section{F. Estimate for $\sqrt{\alpha} e^{2 v}$.}

Lemma 9.3. $\sqrt{\alpha} e^{2 v}$ is uniformly bounded on $\left(t_{f}, t_{i}\right]$.

Proof. It follows from equations (249) and (250) that

$$
\partial_{t}\left(\sqrt{\alpha} e^{2 v}\right) \geq 0
$$

We will use this bound in order to estimate the terms containing $\alpha e^{2 v}$ in the right-hand side of (250). This will follow from the next two lemmas.

9G. Estimate for $\int_{[0,1]}\left|\left(\sqrt{\alpha} e^{v}\right)_{\theta}\right| d \boldsymbol{\theta}$. Let $e^{\beta}=\sqrt{\alpha} e^{v}$. Equation (251) can now be written as

$$
\beta_{\theta}=-8 \pi \sqrt{\alpha} \int_{\mathbb{R}^{3}} f v_{1} \gamma^{-1 / 2} d v_{1} d v_{2} d v_{3}
$$

Lemma 9.4. $\int_{[0,1]}\left|\beta_{\theta}\right| d \theta$ is bounded on $\left(t_{f}, t_{i}\right]$. In particular, there exists a bound independent of $t \in\left(t_{f}, t_{i}\right]$ on the difference between the maximum and the minimum of $\beta(t, \cdot)$. 
Proof. From (263), we have

$$
\left|\beta_{\theta}\right| \leq 8 \pi \sqrt{\alpha} \int_{\mathbb{R}^{3}} f v_{1} \gamma^{-1 / 2} d v_{1} d v_{2} d v_{3} \leq 8 \pi \int_{\mathbb{R}^{3}} f v_{0} \gamma^{-1 / 2} d v_{1} d v_{2} d v_{3} \leq \frac{v_{t}}{\sqrt{\alpha}},
$$

where we have used the fact that $\sqrt{\alpha}\left|v_{1}\right| \leq v_{0}$ from the mass shell relation to obtain the second inequality and (249) to obtain the third.

Dividing (264) by $t$ and integrating $v_{t} / \sqrt{\alpha}$ over $[0,1]$, we obtain a bound on $\int_{[0,1]}\left|\beta_{\theta}\right| d \theta$ from the bounds on $t$ and $E$.

9H. Control of $\alpha$ along special curves. Similar to Section 7H, we now prove:

Lemma 9.5. $\min _{[0,1]} \alpha(t, \cdot)$ is bounded on $\left(t_{f}, t_{i}\right]$.

Proof. From the definition of $E$ and from (249),

$$
8 \pi \int_{[0,1]} \int_{\mathbb{R}^{3}} f \gamma^{-1 / 2}\left|v_{0}\right| d v_{1} d v_{2} d v_{3} d \theta \leq t E(t) .
$$

Since $\sqrt{\alpha}\left|v_{1}\right| \leq v_{0}$, we obtain

$$
\min _{[0,1]}(\sqrt{\alpha}) \int_{[0,1]} \int_{\mathbb{R}^{3}} f\left|v_{1}\right| \gamma^{-1 / 2} d v_{1} d v_{2} d v_{3} d \theta \leq \frac{t E(t)}{8 \pi} \leq A
$$

for some constant $A$ depending on the bound on $E$. However from Lemma 9.1, we have

$$
\delta \leq \int_{[0,1]} \int_{\mathbb{R}^{3}} f\left|v_{1}\right| \sqrt{\gamma}^{-1} d v_{1} d v_{2} d v_{3},
$$

for some $\delta>0$. Therefore $\min _{[0,1]}(\sqrt{\alpha}) \leq A / \delta$.

This concludes the proof of Lemma 9.3.

As in Corollary 1 of Section 7H, we obtain:

Corollary 4. There exists $\bar{\theta}$ such that $\alpha(t, \bar{\theta})$ is bounded on $\left(t_{p}, t_{i}\right]$.

9I. Estimate for $e^{2 \beta}$.

Lemma 9.6. $e^{2 \beta}=\alpha e^{2 v}$ is uniformly bounded on $\left(t_{f}, t_{i}\right] \times[0,1]$.

Proof. This follows from Corollary 4 and Lemmas 9.3 and 9.4 by an argument similar to the one given for the proof of Lemma 7.7.

9J. Estimates for the support of $f$. Let

$$
u_{1}=\sqrt{\alpha} v_{1}=\frac{\sqrt{\alpha} e^{v}}{\sqrt{t}} w
$$

and define $\bar{u}_{1}$ by

$$
\bar{u}_{1}(t)=\sup \left\{\left|u_{1}\right|: \exists(\theta, L) \text { such that } f\left(t, \theta, \frac{u_{1}}{\sqrt{\alpha}}, L\right) \neq 0\right\}
$$

Lemma 9.7. $\bar{u}_{1}$ is uniformly bounded on $\left(t_{f}, t_{i}\right]$. 
Proof. The characteristic equation for $u_{1}$ associated with the Vlasov equation written (248) in terms of the coordinates $\left(t, \theta, u_{1}, L\right)$ gives

$$
\frac{d\left(u_{1}^{2}\right)}{d s}=\frac{\alpha_{t}}{\alpha} u_{1}^{2}+\frac{2 \sqrt{\alpha} u_{1}}{v_{0}} \frac{e^{2 \beta}}{t} \beta_{\theta}(1+L / t) .
$$

The transformation (268) from $w$ to $u_{1}$ will avoid the difficulty arising from the term containing $\beta_{\theta}$ in (248). Indeed, this term contains the factor $\sqrt{1+w^{2}+L / t}$, which depends in $w$ in a not completely trivial way. On the other hand, having $v_{0}$ at the denominator of the last term in the right-hand side of (270) will enable us to easily estimate this term.

Let us first estimate the factor $\alpha_{t} / \alpha$ appearing in the first term of the right-hand side of (270). From (250) and the bounds on $e^{\beta}$ obtained previously, we have, for appropriate constants $C$ and $A^{\prime}$,

$$
\begin{aligned}
\left|\frac{\alpha_{t}}{\alpha}\right| & \leq C+16 \pi \alpha^{3 / 2} e^{2 v} \int_{\mathbb{R}^{3}} \frac{f\left(t^{-1}+L t^{-2}\right)}{\left|v_{0}\right|} \gamma^{-1 / 2} d v_{1} d v_{2} d v_{3} \\
& \leq C+C^{\prime} \sqrt{\alpha} \int_{-\overline{u_{1}}}^{\overline{u_{1}}} \int_{-X}^{X} \frac{f\left(t^{-1}+L t^{-2}\right)}{\left|v_{0}\right|} \frac{d u_{1}}{\sqrt{\alpha}} \pi d L \\
& \leq C+C^{\prime \prime} F\left(\frac{1}{t_{f}}+\frac{X}{t_{f}^{2}}\right) \int_{-\overline{u_{1}}}^{\overline{u_{1}}} \frac{d u_{1}}{\left|v_{0}\right|} \\
& \leq C+A \int_{-\bar{u}_{1}}^{\bar{u}_{1}} \frac{d u_{1}}{\sqrt{1+t e^{-2 \beta} u_{1}^{2}}} \\
& \leq C+A\left[e^{\beta} t^{-1 / 2} \ln \left(u_{1}+\sqrt{e^{2 \beta} / t+u_{1}^{2}}\right)\right]_{-\bar{u}_{1}}^{\bar{u}_{1}} \\
& \leq C+A^{\prime}\left(\ln \left(\bar{u}_{1}+\sqrt{e^{2 \beta} / t+\bar{u}_{1}^{2}}\right)+e^{-1}\right) .
\end{aligned}
$$

We now estimate the second term on the right-hand side of (270). First note that the mass shell relation written in terms of $u_{1}$ reads as

$$
v_{0}=-\sqrt{\frac{\alpha}{t} e^{2 v}+u_{1}^{2}+\frac{\alpha e^{2 v}}{t^{2}} \gamma^{a b} v_{a} v_{b}}
$$

and thus, we have $\left|u_{1}\right| /\left|v_{0}\right|<1$. Moreover, from (263), we have

$$
\sqrt{\alpha} \beta_{\theta} \leq 8 \pi^{2} F X \bar{u}_{1}^{2}
$$

Integrating (270) and using the estimates (273) and (271), we obtain an inequality of the form

$$
u_{1}^{2}(t) \leq A+B \int_{t}^{t_{i}} u_{1}^{2}(s) \ln \left(1+\bar{u}_{1}^{2}\right)(s) d s+C \int_{t}^{t_{i}} \bar{u}_{1}^{2}(s) d s,
$$

for some positive constants $A, B$ and $C$. It follows from the last line, as in (101)-(103), that $\bar{u}_{1}$ is uniformly bounded. 


\section{K. Estimates for $\alpha, \beta, v$ and $\beta_{\theta}$.}

Lemma 9.8. $\alpha, \beta, v$ and $\beta_{\theta}$ are uniformly bounded on $\left(t_{f}, t_{i}\right] \times[0,1]$.

Proof. This follows easily from the Einstein equations since the right-hand sides of equations (249), (250) and (251) contain only quantities that have been shown to be bounded.

9L. Estimates for the derivatives of $f, \alpha_{\theta}, v_{\theta}$ and higher-order estimates. This follows by standard arguments, which can be found for instance in [Weaver 2004].

9M. The conclusion. Since all metric functions, the Vlasov field and all their derivatives have been shown to be uniformly bounded, the assumptions of Proposition 2 have been retrieved. In particular, the maximal Cauchy development cannot have $t_{0}>0$, which concludes the proof of Theorem 3 .

\section{Comments and open questions}

10A. Weaver's estimate for Vlasov matter. The result of Theorem 3 was obtained in [Rein 1996; Tchapnda 2004] under a small data assumption. The main difference in our analysis which enables us to remove this smallness assumption, is to use, following [Weaver 2004], the presence of the Vlasov field to obtain a lower bound on one of the matter terms (see Lemma 9.1). It would be interesting to see if this estimate could be applied in other geometries and what would be the consequences.

Let us also note that if we couple the Einstein-Vlasov system to extra matter fields, a statement analogous to Lemma 9.1 would certainly be true if the extra matter fields satisfy the strong energy condition. For instance, the results of Theorems 1 and 3 can certainly be extended to include a massless scalar field.

10B. Theorem 2 and the hierarchization of the equations. The proof of Theorem 2 is based on the recovery of the lower bound on the energy quantities $E_{h}$ and $E_{g}$. In the vacuum case, this lower bound is obtained directly from the monotonicity of $E_{g}$. However, this monotonicity is unstable to any perturbation in the setting of the problem, such as the introduction of matter or of a positive cosmological constant.

Our strategy has been to prove that, while $E_{h}$ is not necessarily monotone, one can recover a monotonicity for another energy, namely $E_{A}$, which controls $E_{h}$ from below and thus is sufficient to obtain the required lower bound on $E_{h}$. Since $E_{A}$ is the energy associated with the wave equation for $A$ only, while $E_{h}$ is associated for the system of equations for $(U, A)$, this shows that, in the contradiction setting that we have deployed, a certain hierarchy in the evolution equations appears, in the sense that one may first focus on the evolution equation for $A$ and extract information from it, which we then reintroduce in the whole system.

Let us also note that not all estimates derived during the proof of Theorem 2 require the contradiction setting of Section 8Q. In particular, in Section 8J, we have proven a new estimate for $T^{2}$-symmetric spacetimes which might be useful in a further study of these solutions.

10C. Antitrapped initial data. One of the common features of $T^{2}$-symmetric and $k=-1$ surfacesymmetric spacetimes is the antitrapping of the orbits of symmetry. This property arises from the positivity of the Hawking mass (excluding the flat case) and the fact that the orbits of symmetry have nonpositive curvature. The positivity of the Hawking mass is itself a consequence of the topology of Cauchy surfaces 
and of the Einstein equations, especially the Raychaudhuri equations. The proofs of the positivity of the Hawking mass and of antitrapping for vacuum $T^{2}$ symmetry and for $k \leq 0$ surface-symmetric spacetimes with Vlasov matter or with a massless scalar field were first obtained in [Chruściel 1990] and [Rendall 1995]. In [Rendall 1997], the results on $T^{2}$-symmetry were extended to the nonvacuum cases where local $T^{2}$-symmetry only is assumed. In order to improve our understanding of the structure of cosmological singularities, it would be interesting to try to generalize these results. One might ask for instance the following question. Assume that $\Sigma$ is a compact Cauchy surface of a given spacetime satisfying the vacuum Einstein equations such that there exist a diffeomorphism $\phi$ between $\Sigma$ and $S^{1} \times \mathscr{R}$ where $\mathscr{R}$ is a compact surface. Assume moreover that for every point $\theta \in S^{1}, \phi^{-1}(\{\theta\} \times \mathscr{R})$ has nonpositive curvature. Is it then true that $\phi^{-1}(\{\theta\} \times \mathscr{R})$ is necessarily trapped or antitrapped?

10D. Strong cosmic censorship in polarized $\boldsymbol{T}^{\mathbf{2}}$-symmetric spacetimes. Theorems 1,2 and 3 complete our understanding of the value of $t_{0}$ for $T^{2}$-symmetric and surface-symmetric spacetimes, as can be observed in Table 2, and we should therefore focus our attention to the remaining, very difficult, open problems presented in Table 1. One of the first questions to consider is that of strong cosmic censorship for vacuum polarized $T^{2}$-symmetric spacetimes with $\Lambda=0$. While it is likely that the dynamics of these spacetimes are very different from those of general vacuum $T^{2}$-symmetric spacetimes, they are the simplest examples of vacuum inhomogeneous cosmological models where, writing the Einstein equations in areal coordinates, the constraint equations do not decouple from the evolution equations, as can be seen by removing the terms involving $A$ in (104)-(110).

10E. Future causal geodesic completeness of $T^{2}$-symmetric and $k=-1$ surface-symmetric spacetimes. By the arguments of [Dafermos and Rendall 2005], (nonflat) $T^{2}$-symmetric and $k=-1$ surfacesymmetric spacetimes are future inextendible. In the Gowdy case, where a complete understanding of the asymptotics has been obtained [Ringström 2004], and in the $k=-1$ surface-symmetric case with either small data [Rein 2004] or $\Lambda>0$ [Tchapnda and Rendall 2003], future geodesic completeness has also been proven. More generally, we have the following conjecture:

Conjecture 1. Let $(M, g)$ be the maximal Cauchy development of $T^{2}$-symmetric or $k=-1$ surfacesymmetric initial data in the vacuum or with Vlasov matter and with $\Lambda \geq 0$. Assume (M, $g$ ) is nonflat. Denote by $t$ the area of the orbits of symmetry and orient $(\mathcal{M}, g)$ by $\nabla t$. Then $(\mathcal{M}, g)$ is future causally complete.

10F. The past boundary of $\tilde{2}$. One might also consider the following question about the structure of singularities in $T^{2}$-symmetric or $k=-1$ surface-symmetric spacetimes. Let $\tilde{2}$ be the universal cover of the quotient by the group orbits of the maximal Cauchy development. It is possible to draw a Penrose diagram of $\tilde{\mathscr{Q}}$, by introducing bounded double null coordinates on $\tilde{\mathscr{2}}$ and then regarding $\tilde{\mathscr{2}}$ as a bounded subset of $\mathbb{R}^{1+1}$. In the case of vacuum nonflat $T^{3}$-Gowdy initial data with $\Lambda=0$, it is then a well known fact that its past boundary is spacelike with respect to the causality of $\mathbb{R}^{1+1}$ and thus the Penrose diagram takes the following form:

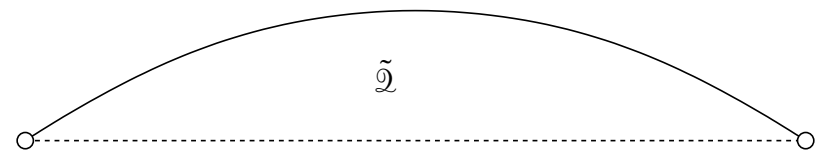


On the other hand, for nongeneric vacuum $T^{2}$-symmetric spacetimes ${ }^{26}$ with $t_{0}>0$, the past boundary is null:

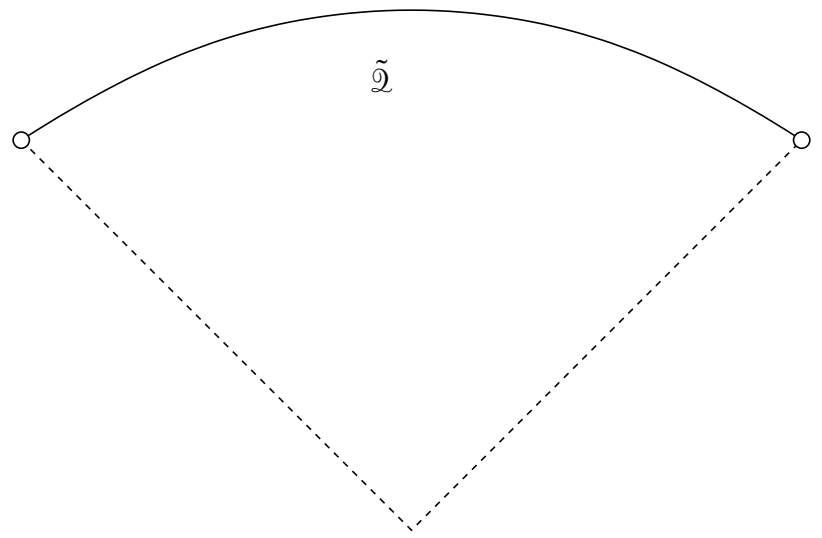

It is natural to ask where the general case stands compared to these two particular cases, whether the past boundary is spacelike, null or neither.

\section{Appendix A. Initial data and constraint equations for the Einstein and Einstein-Vlasov systems}

We present below the constraint equations of the Einstein-Vlasov system. To obtain the constraint equations in the vacuum case, it suffices to replace all matter terms (i.e., all terms containing $\hat{f}$ ) by zero.

Recall that a smooth initial data set for the Einstein-Vlasov system is a quadruplet $(\Sigma, h, K, \hat{f})$ such that:

(1) $\Sigma$ is a smooth 3-dimensional manifold,

(2) $h$ is a smooth Riemannian metric on $\Sigma$,

(3) $K$ is a smooth symmetric 2-tensor on $\Sigma$,

(4) $\hat{f}$ is a smooth function defined on the tangent bundle of $\Sigma$,

(5) $(\Sigma, h, K, \hat{f})$ satisfies the constraint equations

$$
\begin{aligned}
R^{(3)}-K_{a b} K^{a b}+(\operatorname{tr} K)^{2} & =16 \pi \rho+2 \Lambda, \\
\nabla_{a}^{(3)} K_{b}{ }^{a}-\nabla_{b}^{(3)}(\operatorname{tr} K) & =8 \pi j_{b},
\end{aligned}
$$

where $\nabla^{(3)}$ and $R^{(3)}$ denote the Levi-Civita and the Ricci curvature scalar of $h$ and $\rho$ and $j_{b}$ are given by

$$
\begin{aligned}
\rho & =\int_{\mathbb{R}^{3}} \hat{f}\left(1+p^{a} p_{a}\right)^{1 / 2} \sqrt{h} d p^{1} d p^{2} d p^{3}, \\
j_{a} & =\int_{\mathbb{R}^{3}} \hat{f} p_{a} \sqrt{h} d p^{1} d p^{2} d p^{3},
\end{aligned}
$$

where it has been assumed in the above definitions that, if $\pi_{\Sigma}$ denotes the natural projection from $T \Sigma$ to $\Sigma$, then $\left(p^{1}, p^{2}, p^{3}\right)$ are global coordinates on $\pi_{\Sigma}^{-1}(x)$ for any $x \in \Sigma$.

\footnotetext{
${ }^{26}$ See the appendix of [Smulevici 2008], for instance.
} 


\section{Appendix B. Surface-symmetric spacetimes in areal coordinates}

We present in this appendix a change of coordinates and parametrization of the metric which brings the metric (7) from the usual parametrization:

$$
d s^{2}=-e^{2 \mu(r, \theta)} d r^{2}+e^{2 \lambda(r, \theta)} d \theta^{2}+r^{2} \gamma_{a b} d x^{a} d x^{b}
$$

We define the new time coordinate by $t=r^{2}$. The metric now takes the form

$$
d s^{2}=-\frac{e^{2 \mu}}{4 t} d t^{2}+e^{2 \lambda} d \theta^{2}+t \gamma_{a b} d x^{a} d x^{b} .
$$

We can then define the functions $\alpha$ and $v$ by

$$
\begin{aligned}
e^{2 \lambda} & =\frac{e^{2 v}}{t}, \\
e^{2 \mu} & =4 \alpha e^{2 v} .
\end{aligned}
$$

in order to obtain the metric in the form (7).

\section{Appendix C. From symmetric initial data to symmetric spacetimes}

We recall in this section that the symmetries of initial data are transmitted to the maximal Cauchy development. For the proofs in the vacuum case and a more exhaustive treatment of these questions, we refer the reader to the classical work of Chruściel [Chruściel 1991]. We will write the theorems in the vacuum case for simplicity.

First, we recall that Killing data leads to Killing vector fields:

Proposition 5. Let $(\Sigma, h, K)$ be a vacuum initial data set for the Einstein equations. Assume that there exists a smooth vector field $Y$ such that

$$
\mathscr{L}_{Y} h=\mathscr{L}_{Y} K=0
$$

Let $(M, g)$ denote the maximal Cauchy development of $(\Sigma, h, K)$ as in the statement of the theorem of Section 3 and let $\phi: \Sigma \rightarrow M$ be the corresponding embedding. Then there exists a smooth vector field $X$ on $M$ such that

$$
\mathscr{L}_{X} g=0, \quad X_{\mid \phi(\Sigma)}=\phi_{*}(Y) .
$$

Proposition 6. Let $(\Sigma, h, K)$ be a vacuum initial data set for the Einstein equations. Assume that there exists a topological group $G$ and a smooth action of $G$ by isometries on $(\Sigma, h, K)$, that is, a map

$$
\phi: G \times \Sigma \rightarrow \Sigma, \quad(q, p) \mapsto \phi_{q}(p) \quad \text { such that } \phi_{g}^{*} h=h \quad \text { and } \quad \phi_{g}^{*} K=K .
$$

Let $(M, g)$ denote the maximal Cauchy development of $(\Sigma, h, K)$ as in the statement of the theorem of Section 3 and let $i$ be the corresponding embedding of $\Sigma$ in $M$. There exists an action $\psi$ of $G$ on $M$,

$$
\psi: G \times M \rightarrow M, \quad(q, p) \mapsto \psi_{q}(p),
$$

such that, for all $q \in G$,

$$
\psi_{q}^{*} g=g, \quad \psi_{q} \circ i=i \circ \phi_{g}
$$




\section{Acknowledgements}

I would like to thank Mihalis Dafermos for his consistent support, his encouragements, his precious help and for introducing me to the problems addressed in this article. I would also like to thank John Stewart for much useful advice and Gustav Holzegel for many interesting discussions. Finally, I would like to thank Hans Ringström for his comments on a previous version of this text contained in my thesis. The work necessary to prove Theorems 1 and 3 was conducted during my stay in MIT from October to December 2007 and I wish to thank the Department of Mathematics of MIT and the NSF for the help and funding I received during my stay. Finally, I wish to gratefully acknowledge funding from EPSRC.

\section{References}

[Andréasson 1999] H. Andréasson, "Global foliations of matter spacetimes with Gowdy symmetry", Comm. Math. Phys. 206:2 (1999), 337-365. MR 2001k:83010 Zbl 0949.83009

[Andréasson et al. 2003] H. Andréasson, G. Rein, and A. D. Rendall, "On the Einstein-Vlasov system with hyperbolic symmetry", Math. Proc. Cambridge Philos. Soc. 134:3 (2003), 529-549. MR 2004m:83013 Zbl 1028.83012

[Andréasson et al. 2004] H. Andréasson, A. D. Rendall, and M. Weaver, "Existence of CMC and constant areal time foliations in $T^{2}$ symmetric spacetimes with Vlasov matter", Comm. Partial Differential Equations 29:1-2 (2004), 237-262. MR 2006c:83005 Zbl 1063.35145

[Berger et al. 1997] B. K. Berger, P. T. Chruściel, J. Isenberg, and V. Moncrief, "Global foliations of vacuum spacetimes with $T^{2}$ isometry", Ann. Physics 260:1 (1997), 117-148. MR 98j:83005 Zbl 0929.58013

[Choquet-Bruhat 1970] Y. Choquet-Bruhat, "Theorem of uniqueness and local stability for Liouville- Einstein equations", $J$. Mathematical Phys. 11 (1970), 3238-3243. MR 42 \#4159

[Choquet-Bruhat 1971] Y. Choquet-Bruhat, "Problème de Cauchy pour le système intégro différentiel d'Einstein-Liouville", Ann. Inst. Fourier (Grenoble) 21:3 (1971), 181-201. MR 49 \#2018 Zbl 0208.14303

[Choquet-Bruhat and Geroch 1969] Y. Choquet-Bruhat and R. Geroch, "Global aspects of the Cauchy problem in general relativity”, Comm. Math. Phys. 14 (1969), 329-335. MR 40 \#3872 Zbl 0182.59901

[Christodoulou 1999] D. Christodoulou, "On the global initial value problem and the issue of singularities", Classical Quantum Gravity 16:12A (1999), A23-A35. MR 2001a:83010 Zbl 0955.83001

[Chruściel 1990] P. T. Chruściel, “On space-times with U(1) $\times$ U(1) symmetric compact Cauchy surfaces”, Ann. Physics 202:1 (1990), 100-150. MR 91h:83007 Zbl 0727.53078

[Chruściel 1991] P. T. Chruściel, On uniqueness in the large of solutions of Einstein's equations ("strong cosmic censorship”), Proceedings of the Centre for Mathematics and its Applications, Australian National University 27, Canberra, 1991. MR 1143281 (93c:83008

[Chruściel et al. 1990] P. T. Chruściel, J. Isenberg, and V. Moncrief, "Strong cosmic censorship in polarised Gowdy spacetimes”, Classical Quantum Gravity 7:10 (1990), 1671-1680. MR 91i:83042

[Clausen and Isenberg 2007] A. Clausen and J. Isenberg, "Areal foliation and asymptotically velocity-term dominated behavior in $T^{2}$ symmetric space-times with positive cosmological constant”, J. Math. Phys. 48:8 (2007), Art \#082501. MR 2008j:83006

[Dafermos and Rendall 2005] M. Dafermos and A. D. Rendall, "Inextendibility of expanding cosmological models with symmetry”, Classical Quantum Gravity 22:23 (2005), L143-L147. MR 2006k:83031 Zbl 1087.83050

[Dafermos and Rendall 2006] M. Dafermos and A. D. Rendall, "Strong cosmic censorship for $T^{2}$-symmetric cosmological spacetimes with collisionless matter”, preprint, 2006. arXiv gr-qc/0610075

[Dafermos and Rendall 2007] M. Dafermos and A. D. Rendall, "Strong cosmic censorship for surface-symmetric cosmological spacetimes with collisionless matter", preprint, 2007. arXiv gr-qc/0701034

[Fourès-Bruhat 1952] Y. Fourès-Bruhat, “Théorème d'existence pour certains systèmes d'équations aux dérivées partielles non linéaires”, Acta Math. 88 (1952), 141-225. MR 14,756g Zbl 0049.19201 
[Geroch 1972] R. Geroch, "A method for generating new solutions of Einstein's equation. II", J. Mathematical Phys. 13 (1972), 394-404. MR 45 \#9643 Zbl 0241.53038

[Isenberg and Weaver 2003] J. Isenberg and M. Weaver, "On the area of the symmetry orbits in $T^{2}$ symmetric spacetimes", Classical Quantum Gravity 20:16 (2003), 3783-3796. MR 2004f:83049 Zbl 1049.83004

[Moncrief 1981] V. Moncrief, "Global properties of Gowdy spacetimes with $T^{3} \times \mathbf{R}$ topology", Ann. Physics 132:1 (1981), 87-107. MR 83e:83007

[Moncrief and Eardley 1981] V. Moncrief and D. M. Eardley, "The global existence problem and cosmic censorship in general relativity”, Gen. Relativity Gravitation 13:9 (1981), 887-892. MR 83b:83006

[Penrose 1979] R. Penrose, "Singularities and time-asymmetry", pp. 581-638 in General relativity: an Einstein centenary survey, Cambridge University Press, 1979.

[Rein 1996] G. Rein, "Cosmological solutions of the Vlasov-Einstein system with spherical, plane, and hyperbolic symmetry", Math. Proc. Cambridge Philos. Soc. 119:4 (1996), 739-762. MR 96k:83012 Zbl 0851.53058

[Rein 2004] G. Rein, "On future geodesic completeness for the Einstein-Vlasov system with hyperbolic symmetry", Math. Proc. Cambridge Philos. Soc. 137:1 (2004), 237-244. MR 2005e:83010 Zbl 1052.83008

[Rendall 1995] A. D. Rendall, "Crushing singularities in spacetimes with spherical, plane and hyperbolic symmetry", Classical Quantum Gravity 12:6 (1995), 1517-1533. MR 96g:83117 Zbl 0823.53072

[Rendall 1997] A. D. Rendall, "Existence of constant mean curvature foliations in spacetimes with two-dimensional local symmetry", Comm. Math. Phys. 189:1 (1997), 145-164. MR 99c:53075 Zbl 0897.53049

[Ringström 2004] H. Ringström, "On a wave map equation arising in general relativity", Comm. Pure Appl. Math. 57:5 (2004), 657-703. MR 2005h:58049 Zbl 1059.83011

[Ringström 2006] H. Ringström, "Existence of an asymptotic velocity and implications for the asymptotic behavior in the direction of the singularity in $T^{3}$-Gowdy", Comm. Pure Appl. Math. 59:7 (2006), 977-1041. MR 2222842 (2007d:83111 Zbl 1114.83010

[Ringström 2009] H. Ringström, "Strong cosmic censorship in $T^{3}$-Gowdy spacetimes", Ann. of Math. (2) 170:3 (2009), 11811240. MR 2011d:83095 Zbl 1206.83115

[Smulevici 2008] J. Smulevici, "Strong cosmic censorship for $T^{2}$-symmetric spacetimes with cosmological constant and matter”, Ann. Henri Poincaré 9:8 (2008), 1425-1453. MR 2009m:83099 Zbl 1158.83022

[Tchapnda 2004] S. B. Tchapnda, "Structure of solutions near the initial singularity for the surface-symmetric Einstein-Vlasov system”, Classical Quantum Gravity 21:23 (2004), 5333-5346. MR 2005m:83093 Zbl 1082.83025

[Tchapnda and Noutchegueme 2005] S. B. Tchapnda and N. Noutchegueme, "The surface-symmetric Einstein-Vlasov system with cosmological constant”, Math. Proc. Cambridge Philos. Soc. 138:3 (2005), 541-553. MR 2006k:83100 Zbl 1119.83013

[Tchapnda and Rendall 2003] S. B. Tchapnda and A. D. Rendall, "Global existence and asymptotic behaviour in the future for the Einstein-Vlasov system with positive cosmological constant", Classical Quantum Gravity 20:14 (2003), 3037-3049. MR 2004h:83010 Zbl 1056.83005

[Tegankong and Rendall 2006] D. Tegankong and A. D. Rendall, "On the nature of initial singularities for solutions of the Einstein-Vlasov-scalar field system with surface symmetry”, Math. Proc. Cambridge Philos. Soc. 141:3 (2006), 547-562. MR 2008g:83012 Zbl 1160.83308

[Weaver 2004] M. Weaver, "On the area of the symmetry orbits in $T^{2}$ symmetric spacetimes with Vlasov matter", Classical Quantum Gravity 21:4 (2004), 1079-1097. MR 2004j:83033 Zbl 1054.83007

Received 1 Sep 2009. Revised 24 Dec 2009. Accepted 31 Jul 2010.

JACQUES SMULEVICI: jacques.smulevici@math.u-psud.fr

Max Planck Institute for Gravitational Physics (Albert Einstein Institute), Am Mühlenberg 1, D-14476 Potsdam-Golm, Germany

Current address: Laboratoire de Mathématiques, Université Paris-Sud 11, bât. 425, 91405 Orsay, France 


\section{Analysis \& PDE}

pjm.math.berkeley.edu/apde

EDITORS

EDITOR-IN-CHIEF

Maciej Zworski

University of California

Berkeley, USA

BOARD OF EDITORS

\begin{tabular}{|c|c|c|c|}
\hline Michael Aizenman & $\begin{array}{l}\text { Princeton University, USA } \\
\text { aizenman@math.princeton.edu }\end{array}$ & Nicolas Burq & $\begin{array}{l}\text { Université Paris-Sud 11, France } \\
\text { nicolas.burq@math.u-psud.fr }\end{array}$ \\
\hline Luis A. Caffarelli & $\begin{array}{l}\text { University of Texas, USA } \\
\text { caffarel@math.utexas.edu }\end{array}$ & un-Yung Alice Chang & $\begin{array}{l}\text { Princeton University, USA } \\
\text { chang@math.princeton.edu }\end{array}$ \\
\hline Michael Christ & $\begin{array}{l}\text { University of California, Berkeley, USA } \\
\text { mchrist@math.berkeley.edu }\end{array}$ & Charles Fefferman & $\begin{array}{l}\text { Princeton University, USA } \\
\text { cf@math.princeton.edu }\end{array}$ \\
\hline Ursula Hamenstaedt & $\begin{array}{l}\text { Universität Bonn, Germany } \\
\text { ursula@math.uni-bonn.de }\end{array}$ & Nigel Higson & $\begin{array}{l}\text { Pennsylvania State Univesity, USA } \\
\text { higson@ math.psu.edu }\end{array}$ \\
\hline Vaughan Jones & $\begin{array}{l}\text { University of California, Berkeley, USA } \\
\text { vfr@math.berkeley.edu }\end{array}$ & Herbert Koch & $\begin{array}{l}\text { Universität Bonn, Germany } \\
\text { koch@math.uni-bonn.de }\end{array}$ \\
\hline Izabella Laba & $\begin{array}{l}\text { University of British Columbia, Canada } \\
\text { ilaba@math.ubc.ca }\end{array}$ & Gilles Lebeau & $\begin{array}{l}\text { Université de Nice Sophia Antipolis, France } \\
\text { lebeau@unice.fr }\end{array}$ \\
\hline László Lempert & $\begin{array}{l}\text { Purdue University, USA } \\
\text { lempert@math.purdue.edu }\end{array}$ & Richard B. Melrose & $\begin{array}{l}\text { Massachussets Institute of Technology, USA } \\
\text { rbm@math.mit.edu }\end{array}$ \\
\hline Frank Merle & $\begin{array}{l}\text { Université de Cergy-Pontoise, France } \\
\text { Frank.Merle@u-cergy.fr }\end{array}$ & William Minicozzi II & $\begin{array}{l}\text { Johns Hopkins University, USA } \\
\text { minicozz@ math.jhu.edu }\end{array}$ \\
\hline Werner Müller & $\begin{array}{l}\text { Universität Bonn, Germany } \\
\text { mueller@math.uni-bonn.de }\end{array}$ & Yuval Peres & $\begin{array}{l}\text { University of California, Berkeley, USA } \\
\text { peres@stat.berkeley.edu }\end{array}$ \\
\hline Gilles Pisier & $\begin{array}{l}\text { Texas A\&M University, and Paris } 6 \\
\text { pisier@math.tamu.edu }\end{array}$ & Tristan Rivière & $\begin{array}{l}\text { ETH, Switzerland } \\
\text { riviere@math.ethz.ch }\end{array}$ \\
\hline Igor Rodnianski & $\begin{array}{l}\text { Princeton University, USA } \\
\text { irod@math.princeton.edu }\end{array}$ & Wilhelm Schlag & $\begin{array}{l}\text { University of Chicago, USA } \\
\text { schlag@math.uchicago.edu }\end{array}$ \\
\hline Sylvia Serfaty & $\begin{array}{l}\text { New York University, USA } \\
\text { serfaty@ cims.nyu.edu }\end{array}$ & Yum-Tong Siu & $\begin{array}{l}\text { Harvard University, USA } \\
\text { siu@math.harvard.edu }\end{array}$ \\
\hline Terence Tao & $\begin{array}{l}\text { University of California, Los Angeles, USA } \\
\text { tao@math.ucla.edu }\end{array}$ & A Michael E. Taylor & $\begin{array}{l}\text { Univ. of North Carolina, Chapel Hill, USA } \\
\text { met@math.unc.edu }\end{array}$ \\
\hline Gunther Uhlmann & $\begin{array}{l}\text { University of Washington, USA } \\
\text { gunther@math.washington.edu }\end{array}$ & András Vasy & $\begin{array}{l}\text { Stanford University, USA } \\
\text { andras@math.stanford.edu }\end{array}$ \\
\hline Virgil Voicul & $\begin{array}{l}\text { University of California, Berkeley, USA } \\
\text { dvv@math.berkeley.edu }\end{array}$ & Steven Zelditch & $\begin{array}{l}\text { Northwestern University, USA } \\
\text { zelditch@math.northwestern.edu }\end{array}$ \\
\hline
\end{tabular}

PRODUCTION

contact@msp.org

Silvio Levy, Scientific Editor

Sheila Newbery, Senior Production Editor

See inside back cover or pjm.math.berkeley.edu/apde for submission instructions.

The subscription price for 2011 is US \$120/year for the electronic version, and \$180/year for print and electronic. Subscriptions, requests for back issues from the last three years and changes of subscribers address should be sent to Mathematical Sciences Publishers, Department of Mathematics, University of California, Berkeley, CA 94720-3840, USA.

Analysis \& PDE, at Mathematical Sciences Publishers, Department of Mathematics, University of California, Berkeley, CA 94720-3840 is published continuously online. Periodical rate postage paid at Berkeley, CA 94704, and additional mailing offices.

APDE peer review and production are managed by EditFLOW ${ }^{\mathrm{TM}}$ from Mathematical Sciences Publishers.

PUBLISHED BY

mathematical sciences publishers

http://msp.org/

A NON-PROFIT CORPORATION

Typeset in IATEX

Copyright $(2011$ by Mathematical Sciences Publishers 


\section{ANALYSIS \& PDE}

\section{Volume $4 \quad$ No. $2 \quad 2011$}

On the area of the symmetry orbits of cosmological spacetimes with toroidal or hyperbolic 191 symmetry

JACQUES SMULEVICI

On a maximum principle and its application to the logarithmically critical Boussinesq system 247 TAOUFIK HMIDI

Defects in semilinear wave equations and timelike minimal surfaces in Minkowski space

ROBERT JERRARD

Well- and ill-posedness issues for energy supercritical waves

Slim Ibrahim, Mohamed Majdoub and NADER Masmoudi 